\title{
NONLINEAR ATTITUDE CONTROL OF UNDERACTUATED SPACECRAFT
}

\author{
by \\ Alexander Frias \\ Bachelor of Engineering, Ryerson University (2010) \\ Master of Applied Science, Ryerson University (2012)
}

\author{
A dissertation \\ presented to Ryerson University \\ in partial fulfillment of the \\ requirements for the degree of \\ Doctor of Philosophy \\ in the program of \\ Aerospace Engineering
}

Toronto, Ontario, Canada, 2018

(C)Alexander Frias, 2018 


\section{AUTHOR'S DECLARATION}

I hereby declare that I am the sole author of this dissertation. This is a true copy of the dissertation, including any required final revisions, as accepted by my examiners.

I authorize Ryerson University to lend this dissertation to other institutions or individuals for the purpose of scholarly research.

I further authorize Ryerson University to reproduce this dissertation by photocopying or by other means, in total or in part, at the request of other institutions or individuals for the purpose of scholarly research.

I understand that my dissertation may be made electronically available to the public. 


\title{
NONLINEAR ATTITUDE CONTROL OF UNDERACTUATED SPACECRAFT
}

\author{
Alexander Frias \\ Doctor of Philosophy, Aerospace Engineering, Ryerson University, Toronto (2018)
}

\section{ABSTRACT}

This dissertation investigates the nonlinear control of the attitude for an underactuated rigid-body spacecraft system in the body-orbital and inertial frames. The problem involving the stabilization of the body-orbital attitude of an underactuated output-feedback system is examined. Using sliding mode control in conjunction with finite-time nonlinear observer, a novel observer-based control law is rigorously analyzed and proven to achieve attitude convergence. Under time-varying disturbances, inertia matrix uncertainties, and high initial errors, the proposed novel law achieves attitude convergence for three-axis stability and ultimate boundedness within 5 degrees and $0.01 \mathrm{deg} / \mathrm{s}$, for attitude error norm and angular velocity norm, respectively. Next, the attitude control problem is rigorously analyzed in the inertial frame, where the underactuated rigid-body spacecraft system equations of motion are highly nonlinear, and the linearized equations of motion are not controllable. To this end, a generalized velocity-free time-varying state feedback controller is developed to achieve globally exponential stability with respect to the homogenous norm and proven to provide ultimate boundedness of all signals with 5 degrees attitude error norm and $0.5 \mathrm{rad} / \mathrm{s}$ angular velocity error norm. Finally, the inertial frame attitude stabilization problem is treated as an optimal control problem. For this case, the Legendre pseudospectral method is used to discretized the spacecraft dynamics into Legendre-Gauss-Lobatto (LGL) node points, where the Lagrange polynomial interpolation is applied to obtain a suitable candidate optimal control sequence. Model predictive control is used to implement the optimal control in predefined control windows sequentially to achieve three-axis stability for a rest-to-rest maneuver within 0.3 orbit. 


\section{ACKNOWLEDGEMENTS}

This dissertation would not have been possible without the love, motivation, support and generally the positivity of my whole family, specially my loving parents, Eddie and Tess. Thank you. I dedicate this dissertation to you.

Ryerson University have become my second home; many people have become more than just staff members during my academic career. I would like to thank all my professors for contributing to my academic knowledge and wellness. I would like to give my utmost sincere thanks to Mr. Jerry Karpynczyk and Mr. Peter Bradley, as mentors whom I have learned a great deal from. To the Aerospace Engineering Department: Ms. Leah Rogan, Ms. Kim Gallo, Mr. Primoz Cresnik, Dr. Hamid Ghaemi, and Dr. Bo $\operatorname{Tan}^{1}$ for putting a smile on my face whenever I see them. I appreciate your help and enthusiasm. A special thanks to the thesis committee: Dr. John Enright ${ }^{1}$, Dr. Simant Upreti ${ }^{2}$, Dr. Jinjun Shan ${ }^{3}$, Dr. Cheung Poon ${ }^{4}$ and Dr. Ron Vogel $^{5}$. I would like to thank my SSDC/RAC lab colleagues for their contribution to my academic success as pillars of support and de/motivation when there were none: Y. Haddad, K. Chang, D. Braga, Dr. M. Rososhansky, Dr. A. Rahimi, Dr. S. Etemadi, M. Adhikari, C. Wozney, S. Patil, V. Muthusamy, M. Alger, D. Bolatti, E. Gleeson ....and to all lab colleagues I have not listed here. I apologize.

Lastly, I would like to express my deepest gratitude to my academic supervisors/mentors: Dr. Anton H. J. de Ruiter ${ }^{1}$ and Dr. Krishna D. Kumar ${ }^{1}$. Thank you both for your guidance, in academia and in life; your unyielding support that go above and beyond; and your motivation to push through and pursue knowledge. I am very much honored to be your student.

And finally, I would like to thank my fiancée, Lily, for her love, care, support, and most of all, patience. I am thankful.

\footnotetext{
${ }^{1}$ Dissertation Committee Member (Internal Examiner), Department of Aerospace Engineering, Ryerson University.

${ }^{2}$ Dissertation Committee Member (Internal Examiner), Department of Chemical Engineering, Ryerson University.

${ }^{3}$ Dissertation Committee Member (External Examiner), Dept. of Earth and Space Science and Engineering, York University.

${ }^{4}$ Dissertation Committee Chair, Department of Aerospace Engineering, Ryerson University.

${ }^{5}$ Dissertation Committee Chair, Department of Politics \& Public Admin., Ryerson University.
} 


\section{TABLE OF CONTENTS}

AUTHOR'S DECLARATION

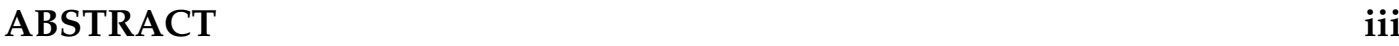

ACKNOWLEDGEMENTS

$\begin{array}{ll}\text { LIST OF TABLES ix } & \text { ix }\end{array}$

LIST OF FIGURES $\quad x$

LIST OF SYMBOLS xii

1 Introduction 1

1.1 Rationale and Motivations . . . . . . . . . . . . . . . . . . 1

1.2 Literature Review . . . . . . . . . . . . . . . . . . . . . 2

1.2.1 Underactuated systems $\ldots \ldots \ldots \ldots \ldots$

1.3 Problem Statement and Research Objectives . . . . . . . . . . . . . . . 5

1.3.1 Body-orbital relative attitude stabilization for an underactuated rigid-body spacecraft system $\ldots \ldots \ldots$

1.3.2 Inertial attitude stabilization for an underactuated rigid-body

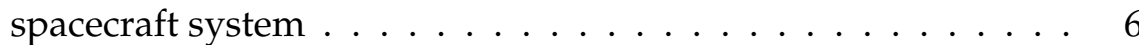

1.3.3 Output feedback control of an Underactuated rigid-body spacecraft system . . . . . . . . . . . . . 6

1.3.4 Lyapunov stability of observer-based nonlinear control system $\quad 7$

1.3.5 Optimal control of underactuated spacecraft using model pre-

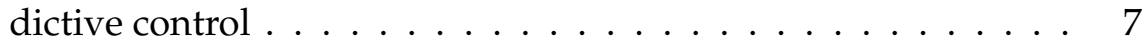

1.4 Research Overview . . . . . . . . . . . . . . . . . . . . 8 
2 Mathematical Preliminaries $\quad 11$

2.1 Vector Notations . . . . . . . . . . . . . . . . . . . . . 11

2.1 .1 Euclidean space . . . . . . . . . . . . . . . . . . . . 11

2.1.2 Vector and matrix norms . . . . . . . . . . . . . . . 11

2.2 Topology . . . . . . . . . . . . . . . . . . . 12

2.2.1 Manifolds . . . . . . . . . . . . . . . . . . 15

2.2.2 Charts and maps . . . . . . . . . . . . . . . . . 15

2.2 .3 Vector fields . . . . . . . . . . . . . . . . . . 16

2.3 Homogeneity of Functions . . . . . . . . . . . . . . . . . 18

2.4 Lyapunov Stability of Systems . . . . . . . . . . . . . . . . . . . . . . 21

2.4 .1 Finite-time stability . . . . . . . . . . . . . 23

2.5 Optimal Control . . . . . . . . . . . . . . . . . . . . 24

2.5.1 The optimal control problem . . . . . . . . . . . . . 25

3 Velocity-Free Nadir-pointing Attitude Stabilization $\quad 27$

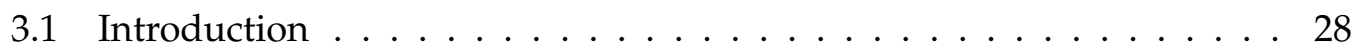

3.2 Problem Formulation . . . . . . . . . . . . . . . . . . . 30

3.2.1 Coordinate frames . . . . . . . . . . . . . . . . 30

3.2.2 Spacecraft attitude kinematics and dynamics . . . . . . . . . 31

3.2.3 Control objective . . . . . . . . . . . . . . . 32

3.3 Design of Underactuated Attitude Control Law . . . . . . . . . . . . . . 33

3.3.1 Control design for unactuated states . . . . . . . . . . . 36

3.3.2 Control design for actuated states $\ldots \ldots \ldots$. . . . . . 41

Full-state feedback control . . . . . . . . . . . . . . . . 42

Velocity-free feedback control . . . . . . . . . . . . . . . 43

3.3.3 Observer design $\ldots \ldots \ldots \ldots \ldots 4 . \ldots \ldots$

3.4 Main Result . . . . . . . . . . . . . . . . . . . . . . . . . . 54

3.5 Numerical Examples . . . . . . . . . . . . . . . . . . 55

3.5.1 Nominal disturbance-free case . . . . . . . . . . . . . 58

3.5.2 Nominal case with disturbances . . . . . . . . . . . . . . . 59

3.5.3 All uncertainties and disturbances . . . . . . . . . . . . . . 60

3.5.4 Effect of $\alpha$ parameter . . . . . . . . . . . . . . 63 
3.6 Chapter Summary . . . . . . . . . . . . . . . . . . . . . 63

4 Inertial Attitude Stabilization using a Generalized Time-Varying Nonlin$\begin{array}{lc}\text { ear Controller } & 66\end{array}$

4.1 Introduction . . . . . . . . . . . . . . . . . . 66

4.2 Problem Formulation . . . . . . . . . . . . . . . . . . 68

4.2.1 Attitude kinematics and dynamics . . . . . . . . . . . . 68

4.2.2 Control objective . . . . . . . . . . . . . . . . . . . . . . . . 69

4.2.3 Control design for unactuated states . . . . . . . . . . . . . 71

4.2.4 Control design for actuated states . . . . . . . . . . 78

4.2 .5 Observer design $\ldots \ldots \ldots$. . . . . . . . . . . 90

4.3 Main Result . . . . . . . . . . . . . . . . . . . . . . . . . . . . . . 94

4.4 Numerical Examples . . . . . . . . . . . . . . . . . . . . . . . 95

4.4.1 Nominal disturbance-free case . . . . . . . . . . . . . . . . . 98

4.4.2 Nominal case with disturbances . . . . . . . . . . . . . . . 98

4.4 .3 All uncertainties and disturbances . . . . . . . . . . . . . . . . 99

4.5 Chapter Summary . . . . . . . . . . . . . . . . . . . . . . . . . 101

5 Model Predictive Control of Underactuated Spacecraft 103

5.1 Introduction . . . . . . . . . . . . . . . . . . . . 103

5.2 Problem Formulation . . . . . . . . . . . . . . . . . . . . 105

5.2.1 Attitude kinematics and dynamics . . . . . . . . . . . 105

5.2 .2 Optimal control . . . . . . . . . . . . . . . . . . . 107

5.2 .3 Pseudospectral discretization . . . . . . . . . . . . . . 108

Time-domain transformation . . . . . . . . . . . . . 109

Lagrange polynomial interpolation . . . . . . . . . . . 109

Differentiation and integration . . . . . . . . . . . 110

5.2 .4 Model predictive control . . . . . . . . . . . . . . . . . 111

5.2 .5 Stability analysis . . . . . . . . . . . . . . . 112

5.3 Numerical Simulations . . . . . . . . . . . . . . . . . . . . . . . . 113

5.4 Chapter Summary . . . . . . . . . . . . . . . . . . . 115 
6 Concluding Remarks $\quad 119$

6.1 Contributions . . . . . . . . . . . . . . . . . . . . . 119

6.2 Conclusions . . . . . . . . . . . . . . . . . . 120

6.3 Future Work . . . . . . . . . . . . . . . . . . . . . . . 121

$\begin{array}{ll}\text { BIBLIOGRAPHY } & 122\end{array}$ 


\section{LIST OF TABLES}

3.1 Summary of parameters $\ldots \ldots \ldots \ldots 6$

4.1 Summary of parameters $\ldots \ldots \ldots \ldots 6$

5.1 MPC Parameters . . . . . . . . . . . . . . . . . . . . . . 114 


\section{LIST OF FIGURES}

1.1 Research overview of the spacecraft attitude control . . . . . . . . 8

1.2 Overview of Chapter $3 \ldots \ldots \ldots \ldots$

1.3 Overview of Chapter $4 \ldots \ldots \ldots \ldots$

1.4 Overview of Chapter $5 \ldots \ldots \ldots$. . . . . . . . . . 10

2.1 Differential maps of a trajectory. . . . . . . . . . . . . . . . 17

3.1 Coordinate Topologies . . . . . . . . . . . . . . . . 30

3.2 Attitude and angular velocity responses in nominal disturbance-free

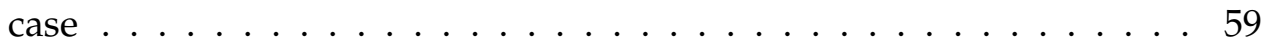

3.3 Attitude and angular velocity responses in nominal case with disturbances ........................... 60

3.4 Control torque in nominal case with disturbances $\ldots . . \ldots 60$

3.5 Attitude and angular velocity responses with inertia matrix uncertainties, non-zero eccentricities and disturbances . . . . . . . . . . . 62

3.6 Effect of $\alpha$ on the transient response . . . . . . . . . . . . 64

3.7 Effect of $\alpha$ on attitude error $\ldots \ldots \ldots$. . . . . . . . . . 64

3.8 Effect of $\alpha$ on the steady-state response . . . . . . . . . . . . 64

4.1 Attitude and angular velocity responses in nominal disturbance-free case .............................. 99

4.2 Attitude and angular velocity responses in nominal case with distur-

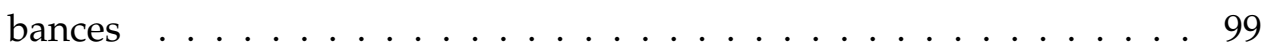

4.3 Control torque in nominal case with disturbances . . . . . . . . . 100

4.4 Attitude and angular velocity responses with inertia matrix uncertainties and disturbances . . . . . . . . . . . . . . . . . . 102 
5.1 Two-RW system topology. . . . . . . . . . . . . . . . . 105

5.2 time domain transformation into computational domain with distinct node points and the corresponding Lagrange interpolation polynomial.110

5.3 Optimal MPC Control Interpolation. . . . . . . . . . . . . . . . . . . 115

5.4 Optimal MPC Control Interpolation: $0 \leq t \leq 0.3$ orbits. . . . . . . . . 115

5.5 Optimal MPC Control Interpolation: $0.7 \leq t \leq 1.0$ orbits. . . . . . . . 116

5.6 MPC continuous dynamics control signals. . . . . . . . . . . . . . 116

5.7 MPC continuous dynamics control signals at $0 \leq t \leq 0.3$ orbit. . . . . 117

5.8 MRP response. . . . . . . . . . . . . . . . . . . 117

5.9 Angular momentum response. . . . . . . . . . . . . . . . . 118 


\section{LIST OF SYMBOLS}

If applicable, the page where the symbol is defined is given in the parentheses. List of common symbols.

\begin{tabular}{|c|c|c|}
\hline$a$ & semi-major axis of the reference orbit & $\mathrm{km}$ \\
\hline $\mathbb{B}_{i}(j)$ & ball of radius $i>0$ centered on point $j$ & \\
\hline$C_{i j}$ & rotation matrix from Frame $j$ to Frame $i$ & \\
\hline$e$ & eccentricity of the reference orbit & \\
\hline$e_{i}$ & unit vector of $i$ th axis & \\
\hline$f(x)$ & vector field of $x$ & \\
\hline $\mathfrak{F}_{I}$ & Earth-centered inertial frame & \\
\hline $\mathfrak{F}_{o}$ & orbital frame & \\
\hline $\mathfrak{F}_{b}$ & spacecraft body-fixed frame & \\
\hline $\mathfrak{F}_{i}$ & coordinate frame of the $i$ th body & \\
\hline$h$ & angular momentum & $\mathrm{N} \cdot \mathrm{m} \cdot \mathrm{s}$ \\
\hline$I$ & identity matrix & \\
\hline$J$ & spacecraft moment of inertia, cost function & $\mathrm{kg} \cdot \mathrm{m}^{2}$ \\
\hline K & control gain & \\
\hline$m, M_{e}$ & mass of spacecraft, mass of the Earth & $\mathrm{kg}$ \\
\hline$p$ & modified Rodrigues parameters & \\
\hline$q$ & inertial unit quaternion & \\
\hline$R$ & orbital radius & $\mathrm{km}$ \\
\hline$S$ & sliding variable & \\
\hline$t$ & temporal parameter & $s$ \\
\hline$V_{x}$ & Lyapunov function $V$ with respect to $x$ & \\
\hline$L_{f} V$ & Lie derivative of $V$ along the direction of the vector field $f$ & \\
\hline$x$ & state parameter & \\
\hline
\end{tabular}


$\epsilon \quad$ body-orbital frame quaternion vector

$\eta \quad$ body-orbital frame quaternion scalar

$\omega \quad$ angular frequency $\quad \operatorname{rad~s}^{-1}$

$\mu \quad$ geocentric gravitational constant of the Earth

$\tau \quad$ external torque

$\omega_{0} \quad$ orbital frame angular velocity $\quad \mathrm{rad} \mathrm{s}^{-1}$

$\|\cdot\|_{p} \quad$ vector $\mathrm{p}$-norm, with $p=\{1,2, \infty\}$

$(\hat{\cdot}) \quad$ estimated parameter

(.) error parameter

$\Delta(\cdot) \quad$ parameter uncertainty

$(\cdot)^{\times} \quad$ skew-symmetry of parameter

$(\cdot)_{k} \quad$ discrete-step of parameter

$\sup (\cdot) \quad$ supremum of parameter

$\inf (\cdot) \quad$ infimum of parameter

$\{\cdots\} \quad$ set of parameters

$\min \{\cdot\} \quad$ minimum of the set

$\max \{\cdot\} \quad$ maximum of the set

$\operatorname{sgn}(\cdot) \quad$ signum of parameter

$\lambda_{\min }(\cdot)$ minimum eigenvalues of parameter

$\lambda_{\max }(\cdot) \quad$ maximum eigenvalues of parameter 
Dedicated to my parents,

Alfredo and Teresita Frias... 


\section{CHAPTER 1}

\section{Introduction}

This dissertation studies the attitude control of a underactuated rigid-body spacecraft system using nonlinear control methods and rigorous analysis of the stability.

The chapter is organized as follows. In the Section 1.1, the rationale behind the research on underactuated control system is explained as well as the motivations behind the methodologies considered. An overview of the literature of relevant topics on the underactuated control systems are covered in Section 1.2. These topics include the works from authors such as Crouch, Khalil, Slotine, and Hughes. Finally, the problem statement and the research objectives are outlined in Section 1.3.

\subsection{Rationale and Motivations}

The development of an attitude control system (ACS) for a spacecraft system to be autonomous, or have complex behaviors such as: capability to analyze mission situations, adapt to changes in environment, system, and uncertainties, or improve performance by learning without human intervention are features that attract technological research and development. If a failure in ACS occurs, in which, leads to a degradation of its actuator(s), can be recovered using a control algorithm and allows the spacecraft system to complete its mission even partially, then it can be considered as a success as opposed to a complete loss. A control algorithm for an underactuated control system can be applied in various ways: ACS redundancy, ACS actuator fault recovery, ACS for an underactuated system by design, reduction in fuel for attitude maneuver or correction. Thus, the development of a novel control design framework for an underactuated system is the motivation of this research. There 
have been many research conducted in the last decade regarding the ACS problem for the underactuated spacecraft system, the following section briefly outlines some significant research and development done in the field.

\subsection{Literature Review}

\subsubsection{Underactuated systems}

Underactuated systems are interesting to study from a theoretical and practical point of view. Control of underactuated systems is considered one of the most active topics within the field of dynamical system due to its potential applications. Underactuation is defined as any system having less control inputs that degrees-of-freedom. Any system, be it mechanical (robotics, mobile systems, marine systems, aerospace systems, flexible systems, locomotive systems, and industrial systems) or biological (animals, human anatomy), can be in a condition of underactuation. The underactuation property of an underactuated system is primarily caused by the following reasons [Xin and Liu, 2014]:

- Natural dynamics of the system (helicopters, underwater vessels, ships, spacecraft, or flexible-link robots)

- Intentional underactuated design (the Acrobot [Spong, 1995], the Pendubot [M. Zhang, 2002], the cart-pole [Aguilar-Ibanez, Mendoza-Mendoza, and Davila, 2014], or the translational oscillation with rotational actuator (TORA) [Gao et al., 2013])

- Reduced actuation or complete actuator failure (complete loss of momentum wheels in a spacecraft, reduced actuation generation in mechanical systems)

The research of the underactuated control systems problem typically fall into one or more of the following categories:

1. Stability and controllability: a problem involving the existence of trajectories that connects initial state to the desired state as well as remain in a prescribed 
region in the state space. This problem essentially pertains to finding the control signals that stabilizes a spacecraft, in which the desired error in the attitude and angular velocity of spacecraft system are zero.

2. Trajectory optimization and planning: a problem involving the computation of feasible trajectory for a given underactuated system that can connect the initial to the desired state in a given finite time. This problem pertains to the finding optimal (or improved) control signals while minimizing certain parameters in a form of a cost function.

3. Tracking: a problem involving the formulation of a feedback control law that can drive the tracking error to zero asymptotically for a given feasible trajectory, at least locally. A particular difficult problem for system that have nonlinearities which involves finding the control signals to stabilize the spacecraft with varying desired trajectory.

4. Set-point regulation: a problem involving the formulation of a feedback control law for given a desired equilibrium state $\mathbf{q}_{d}$ that can make the system state $(\mathbf{q}, \dot{\mathbf{q}})=\left(\mathbf{q}_{d}, 0\right)$ asymptotically stable, at least locally around the trajectory.

The problem of controlling the attitude motion of a rigid-body has been extensively studied in the literature and it is heavily applied in various fields of study, including spacecraft, robotics, and underwater vehicle dynamics. Initial studies in the topic done by Crouch [Crouch, 1984] discussed the possibility of controlling underactuated spacecraft using thrusters or momentum exchanging devices, in which the author concluded that using two independent torques is locally controllable while using one torque yields very serious theoretical and practical difficulties. A study conducted Byrnes and Isidori [Byrnes and Isidori, 1991] further support Crouch's findings and concluded that spacecraft with two controls are locally controllable and locally reachable, but these systems cannot be locally asymptotically stabilized by smooth feedback without using general nonlinear feedback design theory.

Authors in Huang et al. [Huang et al., 2013] proposed the concept of a novel generalized inversion for piecewise-smooth asymptotic stabilization of underactuated rigid body dynamics under two degrees of actuation. This overcame the matrix 
dimensionality and rank which is widely used in solving the control problem with redundant degrees-of-freedom; this was important as the authors concluded that redundancy is a control variable in underactuated system.

Other forms of treatments such as discontinuous state-feedback control and variable structure control strategies for underactuated system had been successfully implemented in literature [Horri and Hodgarts, 2003; Tsiotras and Luo, 2000; Coverstone-Carroll, 1996; Casagrande, Astolfi, and Parisini, 2008]. Control techniques such as sliding mode control in [G. Godard and K.D. Kumar, 2011] for nadir-pointing applications and backstepping have been successful in controlling underactuated systems [G. Godard and K. Kumar, 2010; R. Xu and Ozguner, 2008] and proved effective, resulting in asymptotic stability [Behal et al., 2002]. In this thesis, the nadir-pointing case (relative body-orbital) is also considered, however the strict requirement of higher-order sliding mode imposed in [G. Godard and K.D. Kumar, 2011] is not essential to stabilize the underacted system in case, thus essentially making the control system more robust. In addition, angular velocity is not needed for zero attitude convergence. Model predictive control techniques have also been successfully applied to underactuated spacecraft attitude control [H. Li, Yan, and Shi, 2017; Petersen, Leve, and Kolmanovsky, 2017a]. The authors in [Petersen, Leve, and Kolmanovsky, 2017a] needed to simplify the attitude dynamics model by constructing a linearized model to be implemented as system dynamics for the optimal control computation, leading to an oscillatory error response. In contrast to this thesis, the full nonlinear equations of motion are used in the computation of the optimal control and obtain the Legendre-Gauss-Lobatto optimal nodes, this effectively utilizes the highly nonlinear terms in the dynamics as factors to solving the optimal control leading to exponential convergence. Authors in Narikiyo et al. [Narikiyo, Sahashi, and Misao, 2008] proposed to combine passive velocity field vector control with decoupling vector field in stabilizing underactuated mechanical systems.

In more recent studies regarding the underactuated control problem, are published research by Xiao et al. [Xiao et al., 2014], where the authors developed a velocity-measurement free control scheme in conjunction with nonlinear observer 
for fault-tolerant control of underactuated system in the presence of disturbance and bias [Xiao et al., 2014]. However, the research conducted in this study dealt with a degradation of an actuator on certain axis, in which three-axis actuation was still viable, albeit reduced in certain axis. Contrasted to this thesis, complete absence of actuation in an axis is analyzed with rigor. Authors of Sun et al. [Sun and Fang, 2014] proposed online trajectory planning by incorporating swing-damping terms in a smooth trolley trajectory generation for swing elimination and precise trolley positioning. Authors in Zhang et al. [J. Zhang, Ma, and Meng, 2014] proposed attitude path planning for underactuated spacecraft using two flywheels as actuators. Using an optimal performance index as well as using Gauss Pseudospectral Method (GPM) for optimal path generation the authors were able to generate attitude trajectory for underactuated spacecraft which satisfies the spacecraft maneuver requirements as well as avoid friction influence caused by the flywheels. In comparison to this thesis, the pseudospectral optimal control theory approach is considered to optimize the state trajectory of the spacecraft and implemented using model predictive control, a methodological approach that has not been considered in the literature. Gui et al. [Gui, Vukovich, and S. Xu, 2015] addressed the tracking control of an underactuated spacecraft on the momentum-level set, and developed a control law in conjunction with the Lie group structure of the attitude configuration manifold $S O(3)$ using transverse function (TF) approach developed by Morin and Samson [Morin and Samson, 2009] and analyzed the small-time local controllability of the system in [Gui, Jin, and S. Xu, 2015].

\subsection{Problem Statement and Research Objectives}

The attitude control system for a underactuated rigid-body spacecraft provide several advantages over attitude control systems in fully-actuated spacecraft; namely, weight, cost, and manufacturability. In this dissertation the following problems are analyzed and treated. 


\subsubsection{Body-orbital relative attitude stabilization for an underactuated rigid-body spacecraft system}

The stabilization of the body-orbital relative attitude of a nadir-pointing underactuated rigid-body rigid-body spacecraft system using nonlinear control strategies.

Research objective: The proposed research objective for this problem is the development of the mathematical analysis to achieve stabilization of the body-orbital relative attitude for the underactuated spacecraft and develop a viable nonlinear control strategy using sliding mode control design methodology. To achieve effective stabilization the system dynamics is linearized and utilized to generate control parameters.

\subsubsection{Inertial attitude stabilization for an underactuated rigid-body spacecraft system}

The stabilization of the inertial attitude for a underactuated rigid-body spacecraft system under time-varying disturbances and uncertainties using nonlinear control is examined. Similar to approach to the nadir-pointing case, the inertial case differs due to the fact that the system dynamics are not linearizable, and therefore, limiting the control strategies that may be used.

Research Objective: The proposed research objective for this case is the development of the mathematical analysis to achieve inertial attitude stabilization using a generalized time-varying state feedback controller design.

\subsubsection{Output feedback control of an Underactuated rigid-body spacecraft system}

The problem of achieving attitude convergence of an output feedback system for a underactuated rigid-body spacecraft system is examined. In this case, only angular vector measurements are available and can be used. 
Research objective: The proposed strategy in treating an output feedback system for the underactuated rigid-body spacecraft without angular velocity (henceforth, velocity-free) is to apply a nonlinear observer-based control scheme. This is achieved using the homogeneity of functions.

\subsubsection{Lyapunov stability of observer-based nonlinear control system}

The problem of analyzing the stability of a nonlinear observer-based attitude control system for the rigid-body spacecraft is examined.

Research objective: The proposed research objective is to develop of the mathematical analysis for the stability of the nonlinear observer-based control system using Lyapunov stability theorems and homogeneity of Lyapunov functions. A Lyapunov function that is homogenous is designed and rigorously proven.

\subsubsection{Optimal control of underactuated spacecraft using model predictive control}

The problem of finding a finite-time optimal attitude control sequence for the underactuated rigid-body spacecraft system with two torques is examined. The problem extends to the application of continuous spacecraft dynamics model (as opposed to discretized spacecraft dynamics model), given dynamical constraints.

Research objective: In this problem the proposed research objective is the use pseudospectral optimal control theory. Legendre pseudospectral method of discretization to generate the optimal Legendre-Gauss-Lobatto node points and use Lagrange polynomial interpolation to interpolate an optimal control signals for the model predictive control application to stabilize the underactuated rigid-body spacecraft system. 


\subsection{Research Overview}

The research conducted in this dissertation is described in the following manner. Chapter 1 gives a brief overview of the literature survey on the underactuated spacecraft system and problems pertaining to the research as seen in Figure 1.1 and the motivations and objectives of the research. Chapter 2 outlines the mathematical con-

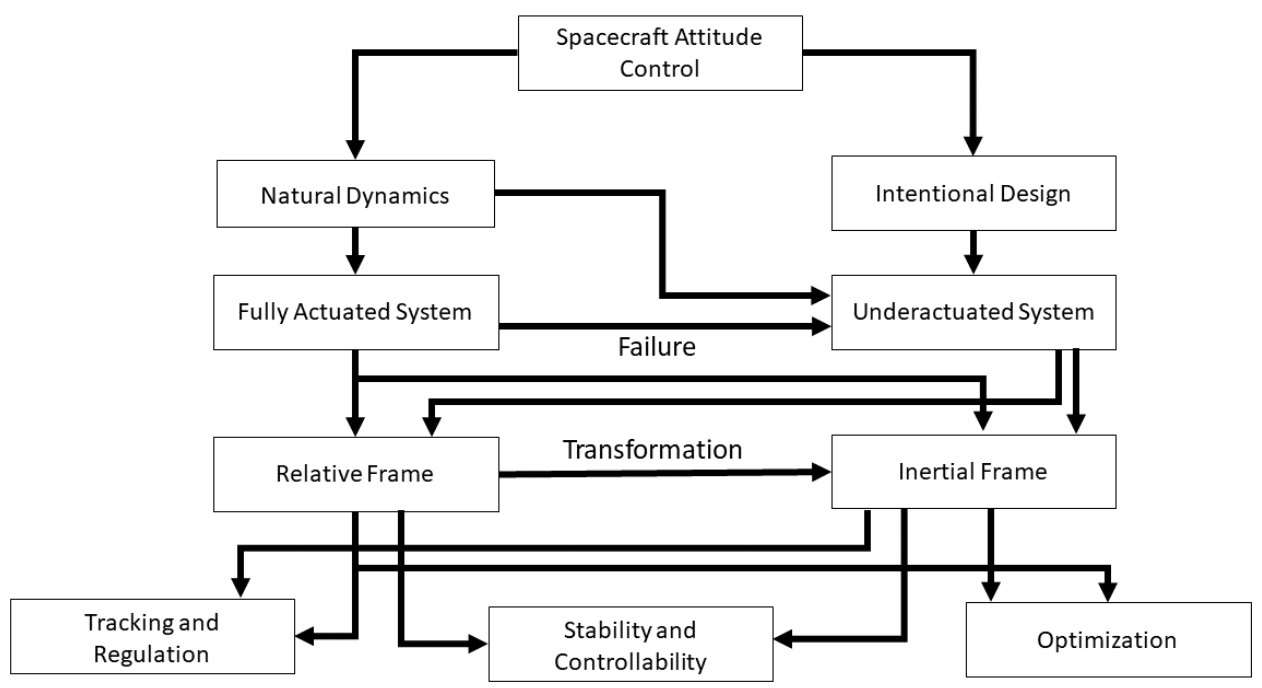

FIGURE 1.1: Research overview of the spacecraft attitude control

cepts to assist the reader in understanding the materials in the subsequent chapters. Chapter 3 examines the attitude stabilization problem of the underactuated spacecraft system without using angular velocity measurements in the body-orbital frame as seen in Figure 1.2. In this chapter, sliding mode control and a nonlinear observer are used to solve the stability and control problems of the underactuated spacecraft system in the relative frame for a nadir-pointing application. Chapter 4 further investigates the problems in Chapter 2 but in the relative inertial frame as seen Figure 1.3. There are several control challenges in the inertial frame, mainly, the methods used in previous chapter is not applicable in the inertial frame, therefore a new method of control is needed. Chapter 5 proposes alternative methods to control the underactuated spacecraft, namely, the use of optimal control as seen in Figure 1.4. Nonlinear model predictive control deals with the optimization of the control input to stabilize the underactuated spacecraft system more effectively. Lastly, Chapter 6 concludes the dissertation with summary and future work. 


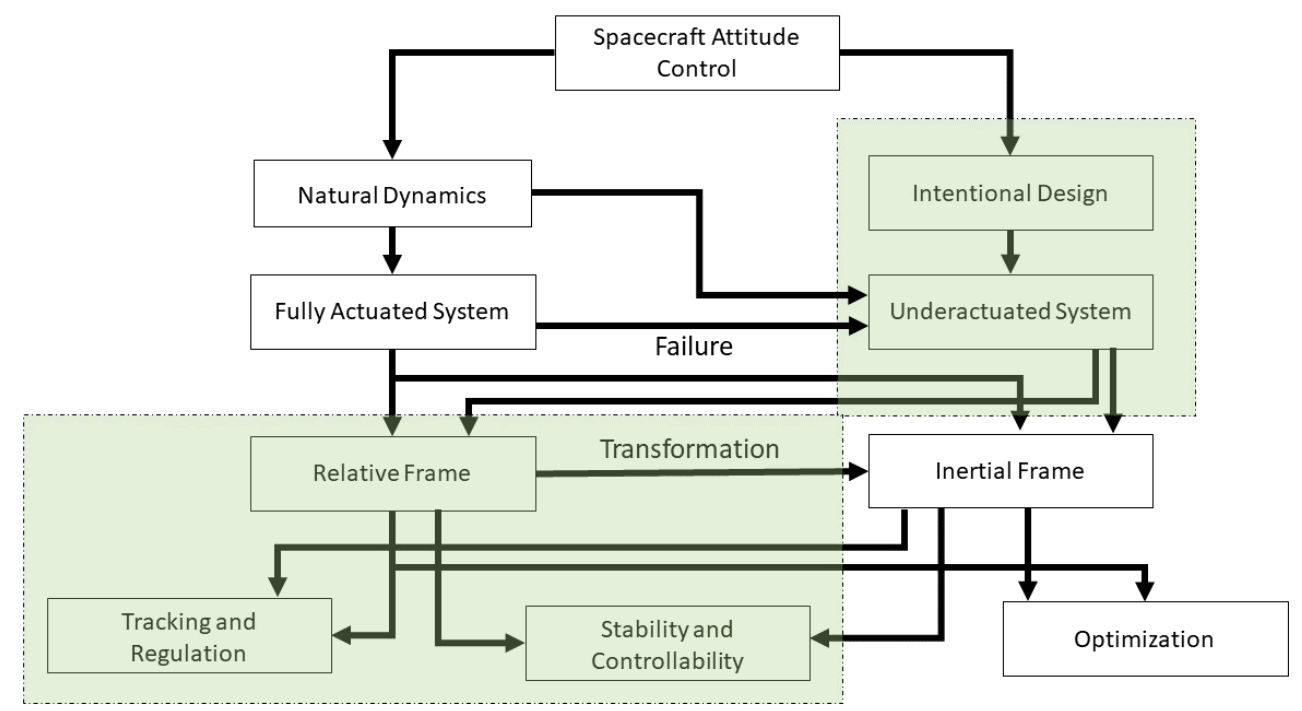

FIGURE 1.2: Overview of Chapter 3

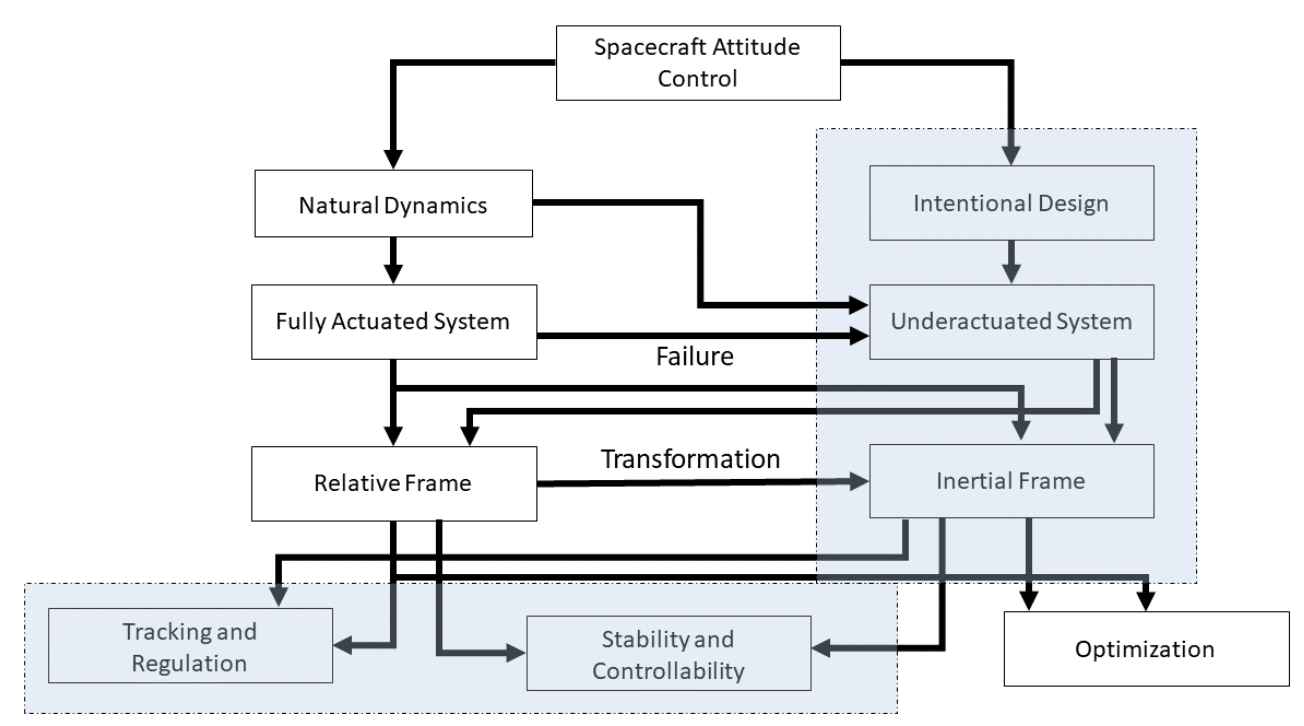

FIGURE 1.3: Overview of Chapter 4 


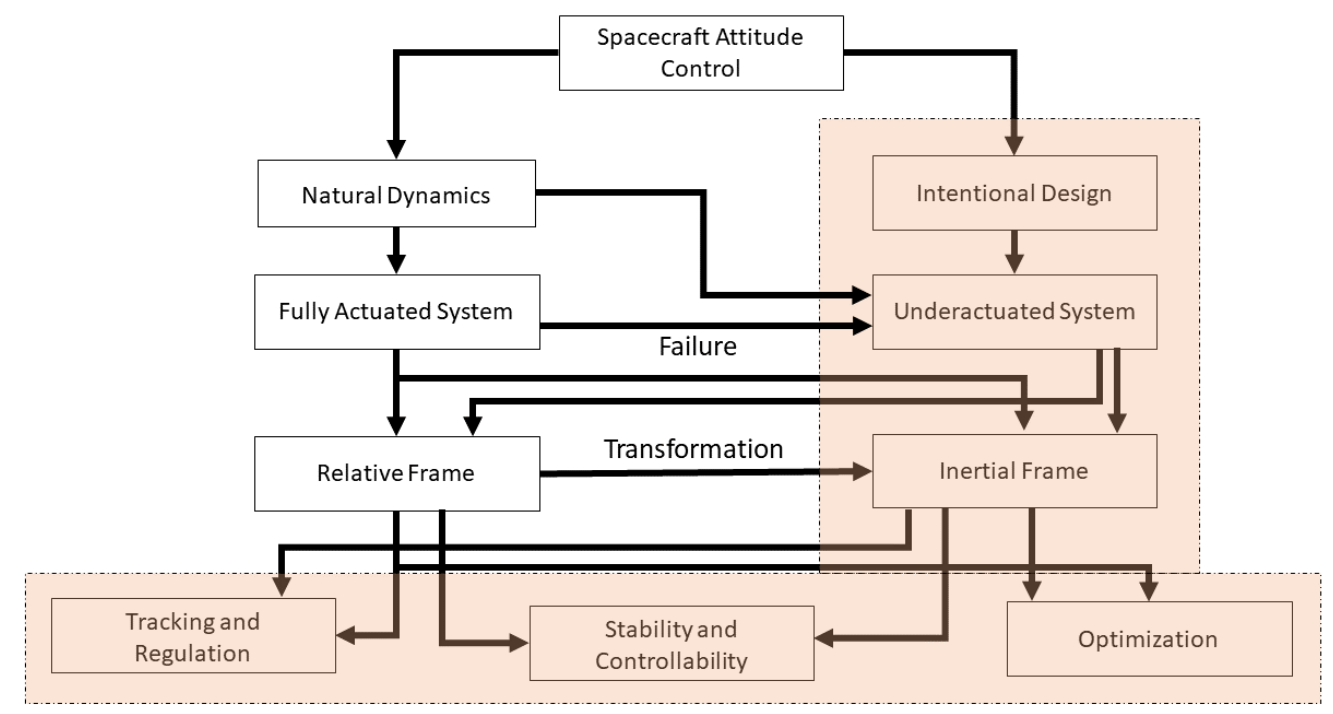

FIGURE 1.4: Overview of Chapter 5 


\section{CHAPTER 2}

\section{Mathematical Preliminaries}

This chapter presents the mathematical background required for the proceeding chapters. The chapter begins with the notations of Euclidean space and norms, a brief review of topics in differential geometry, namely the concepts of topological spaces, differential manifolds, charts and maps, tangent spaces, tangent and vector bundles, and Lie groups. An overview of dynamic stability, finite-time stability, and homogeneity of functions. Finally, we conclude the chapter with topics pertaining to optimal control theory.

\subsection{Vector Notations}

\subsubsection{Euclidean space}

The set of all $n$-dimensional vectors $x=\left[x_{1}, \cdots, x_{n}\right]^{T}$ defines the $n$-dimensional Euclidean space denoted by $\mathbb{R}^{n}$, where $x_{1}, \cdots, x_{n}$ are real valued numbers. We denote by $\mathbb{R}$ to represent the one-dimensional Euclidean space consisting of all real numbers. The set of all non-negative real numbers is denoted by $\mathbb{R}_{+}$. Vectors in $\mathbb{R}^{n}$ can be added by adding their respective components and can be multiplied by a scalar. The inner product of two vectors $x$ and $y$ can be computed as $x^{T} y=\sum_{i=1}^{n} x_{i} y_{i}$. The standard basis vectors for $\mathbb{R}^{3}$ are denoted by $e_{1}=[1,0,0]^{T}, e_{2}=[0,1,0]^{T}$ and $e_{3}=[0,0,1]^{T}$. The identity matrix is denoted by $I$.

\subsubsection{Vector and matrix norms}

The Euclidean norm of a vector $x \in \mathbb{R}^{n}$ is denoted by $\|x\| \geq 0$ for all $x \in \mathbb{R}^{n}$, with $\|x\|=0$ if and only if $x=0$. Given vectors $x, y \in \mathbb{R}^{n},\|x+y\| \leq\|x\|+\|y\|$ 
and $\|a x\|=|a|\|x\|$ for all $a \in \mathbb{R}$. Another property of vector norms is the triangle equality. Consider the class of $p$-norms defined by

$$
\|x\|_{p}=\left(\left|x_{1}\right|^{p}+\cdots+\left|x_{n}\right|^{p}\right)^{1 / p}, \quad 1 \leq p \cdot<\infty
$$

The most commonly used norms are the 1-norm $\|x\|_{1}=\sum_{i=1}^{n}\left|x_{i}\right|$, the $\infty$-norm $\|x\|_{\infty}=\max _{i}\left|x_{i}\right|$ and the Euclidean norm $\|x\|_{2}=\left(x^{T} x\right)^{1 / 2}$. The Hölder inequality is an important result in p-norms, which is given by

$$
\left|x^{T} y\right| \leq\|x\|_{p}\|y\|_{q}, \quad \frac{1}{p}+\frac{1}{q}=1
$$

for all $x, y \in \mathbb{R}^{n}$.

Let $A \in \mathbb{R}^{m \times n}$ be an $m \times n$ matrix of real elements that defines a linear mapping $y=A x$ from $\mathbb{R}^{n}$ into $\mathbb{R}^{m}$, the the induced $p$-norm of $A$ is defined by

$$
\|A\|_{p}=\sup _{x \neq 0} \frac{\|A x\|_{p}}{\|x\|_{p}}=\max _{\|x\|_{p=1}}\|A\|_{p}
$$

and for $p=\{1,2, \infty\}$ is given by

$$
\|A\|_{1}=\max _{j} \sum_{i=1}^{m}\left|a_{i j}\right|, \quad\|A\|_{2}=\left[\lambda_{\max }\left(A^{T} A\right)\right]^{1 / 2}, \quad\|A\|_{\infty}=\max _{i} \sum_{j=1}^{n}\left|a_{i j}\right|
$$

where $\lambda_{\max }\left(A^{T} A\right)$ is the maximum eigenvalue of $\left(A^{T} A\right)$.

\subsection{Topology}

Topology is required in order understand manifold and maps. The definition of a topological space is as follows:

Definition 2.2.1. Topological Space

Denoted by a pair $(S, \mathfrak{O})$, where $S$ is a set and $\mathfrak{O} \subset \mathbf{2}^{S}$ is a collection of open sets that are subsets of $S$ and satisfies the following axioms:

(i) $\varnothing \in \mathfrak{O}$ and $X \in \mathfrak{D}$ is any arbitrary subset of $S$,

(ii) if $A$ is an arbitrary index set and $\left\{\mathcal{O}_{a}\right\}_{a \in A} \subset \mathfrak{O}$ is an arbitrary collection of open sets, then its union $\cup_{a \in A} \mathcal{O}_{a} \in \mathfrak{O}$, 
(iii) if $\mathcal{O}_{1}, \mathcal{O}_{2} \in \mathfrak{O}$, then the intersection $\mathcal{O}_{1} \cap \mathcal{O}_{2} \in \mathfrak{O}$.

Thus, for given a set $S$, a collection of subsets $\mathfrak{O}$ that are open sets is a topology on $S$.

Definition 2.2.2. Topology of Sets.

Let $S$ be any given set. Then,

(i) A subset $C \subset S$ is called closed if its complement $S \backslash\{C\}$ is open.

(ii) For point $p \subset S$, a neighborhood of $p$ is an open set $\mathcal{U} \subset \mathfrak{O}$ for which $p \subset \mathcal{U}$.

(iii) A set $A \subset S$, then the interior of $A$ is the subset of $A$ defined by

$$
\operatorname{int}(A)=\bigcup\{\mathcal{O} \in \mathfrak{O} \mid \mathcal{O} \subset A\}
$$

(iv) If $A \subset S$, then a point $p \subset S$ is a limit point of $A$ if, $\mathcal{U} \cap A \neq \varnothing$, for any neighborhood $\mathcal{U}$ of $p$.

(v) A set $A \subset S$, then the closure of $A$ is the subset of $A$ defined by

$$
\operatorname{cl}(A)=\bigcap\{C \mid C \text { is closed and } C \subset A\} .
$$

(vi) If $A \subset S$, then the boundary of $A$ is the subset of $S$ defined by $\mathbf{b d}(A)=\mathbf{c l} \cap$ $\operatorname{cl}(S \backslash\{A\})$.

(vii) A subset $\mathfrak{B} \subset \mathfrak{O}$ is a basis for $\mathfrak{O}$ if, for every $\mathcal{O} \in \mathfrak{O}$, there exist an index set $A$ and a collection of sets $\left\{B_{a}\right\}_{a \in A} \subset \mathfrak{B}$ such that $\mathcal{O}=\cup_{a \in A} B_{a}$. We say in this case that $\mathfrak{B}$ generates $\mathfrak{O}$

(viii) A cover of $(S, \mathfrak{O})$ is a subset $\left\{\mathcal{O}_{a}\right\}_{a \in A} \subset \mathfrak{O}$ with the property that $S=\cup_{a \in A} \mathcal{O}_{a}$.

(ix) A cover $\left\{\tilde{\mathcal{O}}_{\tilde{a}}\right\}_{\tilde{a} \in \tilde{A}}$ is a refinement of a cover $\left\{\mathcal{O}_{a}\right\}_{a \in A}$ if, for every $a \in A$, there exist $\tilde{a} \in \tilde{A}$ such that $\tilde{\mathcal{O}} \subset \mathcal{O}$.

(x) A subset $\left\{\mathcal{O}_{a}\right\}_{a \in A} \subset \mathfrak{O}$ is locally finite if, for each $p \in S$, there is a neighborhood $\mathcal{U}$ such that the set $\{a \in A \mid \mathcal{U} \cap \mathcal{O} \neq \varnothing$ is finite.

(xi) If $A \subset S$, then one defines a topology on $A$ by $\{A \cap \mathcal{O} \mid \mathcal{O} \in \mathfrak{O}\}$. This is called the subspace topology. 
(xii) If $B \subset A \subset S$, then int $_{A}(B)$ denotes the interior of $B$ in the subspace topology on $A$.

Definition 2.2.3. Set Properties.

Let $(S, \mathfrak{O})$ be any arbitrary topological space. Then,

(i) $(S, \mathfrak{O})$ is first-countable if, for every point $p \subset S$, there exist a sequence of neighborhood $\left\{\mathcal{U}_{j}\right\}_{j \in \mathbb{N}}$ of $p$ such that, for any neighborhood $\mathcal{U}$ of $p$, there exist $j \in \mathbb{N}$ with $\mathcal{U}_{j} \subset \mathcal{U}$.

(ii) $(S, \mathfrak{O})$ is second-countable if, it has a countable basis.

(iii) $(S, \mathfrak{O})$ is compact if, for every collection $\left\{\mathcal{O}_{a}\right\}_{a \in A}$ of open sets for which $S=$ $\cup_{a \in A} \mathcal{O}_{a}$, there exist a finite subset $\left\{a_{1}, \ldots, a_{k}\right\} \subset A$ such that $S=\cup_{j=1}^{k} \mathcal{O}_{a_{j}}$.

(iv) A subset $A \subset S$ is compact if $A$ is compact with the subspace topology.

(v) An open set $\mathcal{U} \subset S$ is relatively compact if its closure is compact.

(vi) $(S, \mathfrak{O})$ is Hausdorff if, for each distinct points $p_{1}, p_{2} \in S$, there exist neighborhoods $\mathcal{U}_{1}$ of $p_{1}$ and $\mathcal{U}_{2}$ of $p_{2}$ such that $\mathcal{U}_{1} \cap \mathcal{U}_{2}=\varnothing$.

(vii) $(S, \mathfrak{O})$ is connected if, when $A \in 2^{S}$ has the property that $A$ is both open and closed, then $A \in\{\varnothing, S\}$,

(viii) and it is disconnected if it is not connected.

(ix) $(S, \mathfrak{O})$ is paracompact if its Hausdorff and every cover possesses a locally finite refinement.

(x) A subset $A \subset S$ of a topological space is dense if $\mathbf{c l}(A)=S$.

Given a point $x \in \mathbb{R}^{n}$ and $r>0, B_{r}(x)=\left\{y \in \mathbb{R}^{n}:\|y-x\|<r\right\}$ and $\bar{B}_{r}(x)=\left\{y \in \mathbb{R}^{n}:\|y-x\| \leq r\right\}$ denote the open and closed balls of radius $r>0$ centered on $x$ respectively. Given a differentiable function $V(x): \mathbb{R}^{n} \rightarrow \mathbb{R}$ and vector field $f(x, t): \mathbb{R}^{n} \times \mathbb{R} \rightarrow \mathbb{R}^{n}$, the Lie derivative of $V$ along $f$ is defined by $L_{f} V=(\partial V / \partial x) f(x, t)$. Given a vector $x \in \mathbb{R}^{n}$ and $\alpha>0$, denote $\operatorname{sig}^{\alpha}(x)=$ $\left[\operatorname{sig}^{\alpha}\left(x_{1}\right), \ldots, \operatorname{sig}^{\alpha}\left(x_{n}\right)\right]^{T}$ and $\operatorname{sig}^{\alpha}\left(x_{i}\right)=\left|x_{i}\right|^{\alpha} \operatorname{sgn}\left(x_{i}\right), i=1,2, \ldots, n$, and $\operatorname{sgn}(\cdot)$ is the signum function. The unit sphere in $\mathbb{R}^{n}$ is defined as $\mathbb{S}^{n-1}=\left\{x \in \mathbb{R}^{n}:\|x\|=1\right\}$. 


\subsubsection{Manifolds}

Definition 2.2.4. Topological Manifold and Submanifold.

A topological space $\mathrm{M}$ can be called a $n$-dimensional smooth manifold. A submanifold of $M$ is a subset $B \subset M$ with the property that for every $b \in B$ there is an admissible chart $(U, \phi)$ in $M$ with $b \in U$ which has the submanifold property, namely,

$$
\phi: U \rightarrow E \times F \quad \text { and } \quad \phi(U \cap B)=\phi(U) \cap(E \times\{0\}) .
$$

Definition 2.2.5. Tangent Space.

For a manifold $M$ and $p \in M$ the tangent space to $M$ at $p$ is the set of equivalence class of curves at $p$ :

$$
T_{p} M=\left\{\left[c_{m}\right] \mid c \text { is a curve at } m\right\}
$$

For a subset $A \subset M$, let the disjoint union, $T M \mid A=\cup_{p \in A} T_{m} M$. We call $T M=$ $T M \mid M$ the tangent bundle of $M$. The mapping $\tau_{M}: T M \rightarrow M$ defined by $\tau_{M}\left([c]_{m}\right)=$ $m$ is the tangent bundle projection of $M$.

\subsubsection{Charts and maps}

Definition 2.2.6. Chart.

Suppose $f: M \rightarrow N$, where $M$ and $N$ are $C^{k}$ manifolds ( $f$ maps the set of $M$ into $N)$. We say $f$ is of class $C^{r}, 0 \leq r \leq k$, if for each $p \in M$ and admissible chart $(V, \psi)$ of $N$ with $f(x) \in V$, there is a chart $(U, \phi)$ of $M$ satisfying $p \in U$ and $f(U) \subset V$, and such that the local representative of $f, f(\phi \psi)=\psi \circ f \circ \phi^{-1}$, is of class $C^{r}$.

Proposition 1 (Continuous Mapping). Let $f: M \rightarrow N$ be a continuous map of manifolds. Then $f$ is $C^{r}$ iff the local representatives of $f$ relative to a collection of charts which cover $M$ and $N$ are $C^{r}$. Similarly, if $f: M \rightarrow N$ and $g: N \rightarrow P$ are $C^{r}$ maps, then so is $g \circ f$.

Definition 2.2.7. Diffeomorphism.

A map $f: M \rightarrow N$ where $M$ and $N$ are manifolds is called a $C^{r}$ diffeomorphism if $f$ is of class $C^{r}$, is a bijection, and $f^{-1}: N \rightarrow M$ is of class $C^{r}$. If a diffeomorphism exists between two manifolds, they are called diffeomorphic and a set of class $C^{r}$ diffeomorphism of $M$ is given by $\operatorname{Diff}^{r}(M)$. 
Definition 2.2.8. Higher-order differential maps.

Let $\mathcal{U}$ be any open set subset of $\mathbb{R}^{n}$ and let $f: \mathcal{U} \rightarrow \mathbb{R}^{m}$.

(i) If $\mathbf{D}^{r} f$ exists and is continuous, then $f$ is $r$-times continuously differentiable, or said to be a class $C^{r}$ map.

(ii) $f$ is smooth or of class $C^{\infty}$ if, $f$ is infinitely differentiable, ie., $f$ or class $C^{r}$ is define for each $r \in \mathbb{N}$.

(iii) A bijection of open sets $f: \mathcal{U} \subset \mathbb{R}^{n} \rightarrow \mathcal{V} \subset \mathbb{R}^{m}$ is of class $C^{r}$ and $f^{-1}$ exists and also of class $C^{r}$, then the bijection $f$ is considered a $C^{r}$-diffeomorphism.

A pair $\left(U_{\alpha}, \phi_{\alpha}\right)$ is called a coordinate chart of $M$ at $q \in M$ where $U_{\alpha}$ is an open set of $M$ containing the point $q$, and the homeomorphism $\phi_{\alpha}$ is a bijection from $U_{\alpha}$ to $\phi_{\alpha}\left(U_{\alpha}\right) \subset \mathbb{R}^{n}$ which composes of local coordinate functions $\left(x^{1}(q), \ldots, x^{n}(q)\right)$.

\subsubsection{Vector fields}

Definition 2.2.9. Vector fields.

A vector field in an open set of a manifold $U \subset M$, where $M \subset R^{n}$, is a differentiable map which assigns to each $q \in U$ a vector $w(q) \in T_{q} M$, where $T_{q} M \subset R^{n}$. The vector field $w(q)$ is a differentiable at $q \in U$, for some local parametrization $p(x, y)$ at $q$. If $q=(x, y)$ and $w(q)=(a(x, y), b(x, y))$, the functions $a$ and $b$ are differentiable functions in $U$. Thus, given a vector field $w(q)$, it is logical to see if there exist a trajectory of this field, that is a differentiable parameterized curve $\alpha(t)=(x(t), y(t))$, $t \in I$, such that $\alpha^{\prime}(t)=w(\alpha(t))$. The vector field $w$ determines a system of differential equations,

$$
\frac{d x}{d t}=a(x, y), \quad \frac{d y}{d t}=b(x, y)
$$

and that a solution to the given equations above is a trajectory. The following Theorems are useful pertaining to the (local) existence and uniqueness of solutions [Carmo, 1976].

Theorem 2.1 (do Carmo). [Carmo, 1976] Let $w$ be a vector field in an open set $U \subset R^{n}$. Given $p \in U$, there exists a trajectory $\alpha: I \rightarrow U$ of $w$ such that $\alpha^{\prime}(t)=w(\alpha(t)), t \in I$ with $\alpha(0)=p$. This trajectory is unique if another trajectory $\beta: J \rightarrow U$ with $\beta(0)=p$ agrees with $\alpha$ in $I \cap J$. I and J are open intervals of the line $R$ containing the origin $0 \in R$. 
Theorem 2.2. [Carmo, 1976] Let $w$ be a vector field in an open set $U \rightarrow R^{n}$. For each $p \in U$ there exist a neighborhood $V \subset U$ of $p$, an interval I, and a mapping $\alpha: V \times I \rightarrow U$ such that

(i) For a fixed $p \in V$, the curve $\alpha(q, t), t \in I$, is the trajectory of w passing through $q$, such that

$$
\alpha(q, 0)=q, \quad \frac{\partial \alpha}{\partial t}(q, t)=w(\alpha(q, t))
$$

(ii) the parameterized curve $\alpha$ is differentiable.

This means that the trajectory passing through $q$ "varies differentiably with $q . "$ Geometrically this means that all trajectories which pass, for $t=0$ in a certain neighbourhood $V$ of $q$ may be collected into a single differential map as seen in Figure 2.1. The map $\alpha$ is called the (local) flow of $w$ at $q$.

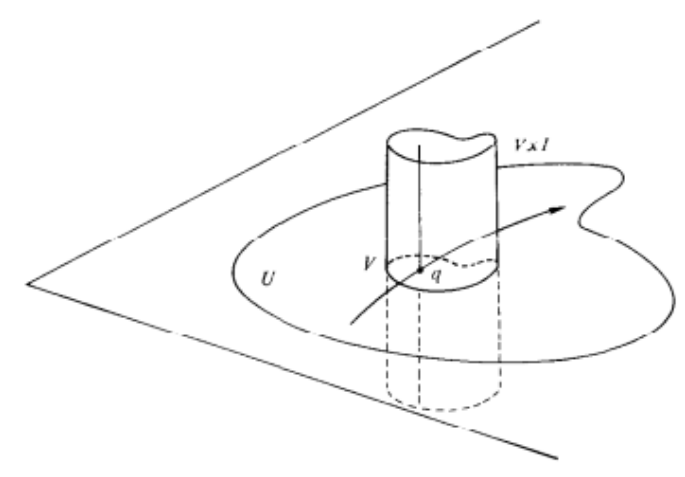

FIGURE 2.1: Differential maps of a trajectory.

Proof. For proof of Theorem 2.1 and Theorem 2.2, please see sections "Lipschitz Conditions" and "Existence and Properties of Solutions of the System" in Hurewicz [Hurewicz, 1952].

Using Theorem 2.1 and Theorem 2.2, the following Lemma can be stated.

Lemma 2.3. Let $w$ be a vector field in an open set $U \rightarrow R^{n}$ and let $p \in U$ be such that $w(p) \neq 0$. Then there exist a neighborhood $W \subset U$ of $p$ and a differentiable function $f: W \rightarrow R$ such that $f$ is constant along each trajectory of $w$ and $d f_{q} \neq 0$ for all $q \in W$. Proof. See do Carmo [Carmo, 1976], pp. 177. 


\subsection{Homogeneity of Functions}

The following definitions are required facts about homogeneity of functions and vector fields.

Definition 2.3.1. Dilation.

Dilation is a mapping depending on the dilation coefficients $r_{i}>0$ for $i=1, \ldots, n$, define $r=\left(r_{1}, \ldots, r_{n}\right) \in \mathbb{R}^{n}$. Then, for any $\lambda>0, x \in \mathbb{R}^{n}$, we define the dilation $\delta_{\lambda}^{r}(x)=\left[\lambda^{r_{1}} x_{1}, \ldots, \lambda^{r_{n}} x_{n}\right]^{T}$ to assign a global diffeomorphism.

Definition 2.3.2. Homogeneity of Functions.

Let $V: \mathbb{R}^{n} \rightarrow \mathbb{R}$, then it is said to be homogeneous of degree $k$ with respect to dilation $\delta_{\lambda}^{r}$ if

$$
V\left(\delta_{\lambda}^{r}(x)\right)=\lambda^{k+r_{i}} V(x)
$$

for all $\lambda>0$ and $x \in \mathbb{R}^{n}$.

Definition 2.3.3. Homogeneity of Vector Fields.

Let $f: \mathbb{R}^{n} \rightarrow \mathbb{R}^{n}$, then it is said to be homogeneous of degree $k$ with respect to dilation $\delta_{\lambda}^{r}$ if

$$
f_{i}\left(\delta_{\lambda}^{r}(x)\right)=\lambda^{k+r_{i}} f_{i}(x)
$$

for all $i=1, \ldots, n, \lambda>0$ and $x \in \mathbb{R}^{n}$, where $f(x)=\left[f_{1}(x), \ldots, f_{n}(x)\right]^{T}$. A system is called homogenous if its vector field is homogenous.

Proposition 2. Let $V: \mathbb{R}^{n} \rightarrow \mathbb{R}$ be homogenous of degree $k$ with respect to $\delta_{\lambda}^{r}$, then $V(0)=0$. Let $f: \mathbb{R}^{n} \rightarrow \mathbb{R}^{n}$ be homogenous of degree $k$ with respect to $\delta_{\lambda}^{r}$, such that $k \neq-r_{i}$ for $i=1, \ldots, n$, then $f(0)=0$.

Proof. We have $V(0)=V\left(\delta_{\lambda}^{r}(0)\right)=\lambda^{k} V(0)$ for any $\lambda>0$, from which it follows that $V(0)=0$. Similarly one finds that $f(0)=0$ provided $k \neq-r_{i}$ for $i=1, \ldots, n$.

Proposition 3. (Rosier) [Rosier, 1992].

Let $V: \mathbb{R}^{n} \rightarrow \mathbb{R}$ be continuously differentiable and homogeneous of degree $\ell$ with respect to $\delta_{\lambda}^{r}$. Then, its partial derivatives satisfy

$$
\frac{\partial V}{\partial x_{i}}\left(\delta_{\lambda}^{r}(x)\right)=\lambda^{\ell-r_{i}} \frac{\partial V}{\partial x_{i}}(x)
$$


for all $i=1, \ldots, n, \lambda>0$ and $x \in \mathbb{R}^{n}$. Furthermore, if $\ell \neq r_{i}$ for $i=1, \ldots, n$, then $\partial V(0) / \partial x=0$.

Proof. By definition,

$$
\frac{\partial V}{\partial x_{i}}\left(\delta_{\lambda}^{r}(x)\right)=\lim _{h \rightarrow 0} \frac{V\left(\lambda^{r_{1}} x_{1}, \cdots, \lambda^{r_{i}} x_{i}+h, \cdots, \lambda^{r_{n}} x_{n}\right)-V\left(\delta_{\lambda}^{r}(x)\right)}{h}
$$

Consider the change of variable $\bar{h}=h / \lambda^{r_{i}}$. This leads to

$$
\begin{aligned}
\frac{\partial V}{\partial x_{i}}\left(\delta_{\lambda}^{r}(x)\right) & =\lim _{h \rightarrow 0} \frac{V\left(\lambda^{r_{1}} x_{1}, \cdots, \lambda^{r_{i}} x_{i}+\bar{h}, \cdots, \lambda^{r_{n}} x_{n}\right)-V\left(\delta_{\lambda}^{r}(x)\right)}{\bar{h} / \lambda^{r_{i}}} \\
& =\lambda^{\ell} \lim _{h \rightarrow 0} \frac{V\left(x_{1}, \cdots, x_{i}+\bar{h}, \cdots, x_{n}\right)-V(x)}{\bar{h} / \lambda^{r_{i}}} \\
& =\lambda^{\ell} \frac{\partial V}{\partial x_{i}}(x)
\end{aligned}
$$

where homogeneity of $V(x)$ has been used in the second step. We see that $\partial V(0) / \partial x=0$ if $\ell \neq r_{i}$ for $i=1, \cdots, n$ as shown similarly to Proposition 1 . This concludes the proof.

Proposition 4. Let $V: \mathbb{R}^{n} \rightarrow \mathbb{R}$ be continuously differentiable and homogeneous of degree $\ell$ with respect to $\delta_{\lambda}^{r}$, and let $f: \mathbb{R}^{n} \rightarrow \mathbb{R}^{n}$ be homogeneous of degree $k$ with respect to $\delta_{\lambda}^{r}$. Then, $L_{f} V(x)$ is homogeneous of degree $\ell+k$ with respect to $\delta_{\lambda}^{r}$. Furthermore, if either $\ell \neq r_{i}, k \neq-r_{i}$ or $\ell+k \neq-r_{i}$ for $i=1, \ldots, n$, then $L_{f} V(0)=0$.

Proof. By definition,

$$
\begin{aligned}
L_{f} V\left(\delta_{\lambda}^{r}(x)\right) & =\sum_{i=1}^{n} \frac{\partial V}{\partial x_{i}}\left(\delta_{\lambda}^{r}(x)\right) f_{i}\left(\delta_{\lambda}^{r}(x)\right) \\
& =\sum_{i=1}^{n} \lambda^{\ell-r_{i}} \frac{\partial V}{\partial x_{i}}(x) \lambda^{k+r_{i}} f_{i}(x), \\
& =\lambda^{\ell+k} \sum_{i=1}^{n} \frac{\partial V}{\partial x_{i}}(x) \lambda_{i}^{f}(x) \\
& =\lambda^{\ell+k} L_{f} V(x)
\end{aligned}
$$

Here, $L_{f} V(x)$ is homogeneous of degree $\ell+k$ with respect to $\delta_{\lambda}^{r}$ follows from Proposition 2. The last statement in the proposition follows from Propositions 1 and 2. This concludes the proof.

Proposition 5. (Bhat et. al.) [S.P. Bhat and Bernstein, 2005]. 
Consider two continuous functions $f_{1}, f_{2}: \mathbb{R}^{n} \rightarrow \mathbb{R}$, homogeneous of degrees $k_{1}, k_{2}$ respectively with respect to dilation $\delta_{\lambda}^{r}$. In addition, suppose that $f_{1}$ is positive definite. Then,

$$
-c_{1} f_{1}^{k_{2} / k_{1}}(x) \leq f_{2}(x) \leq-c_{2} f_{1}^{k_{2} / k_{1}}(x), \forall x \in \mathbb{R}^{n},
$$

where $c_{1}=-\min _{x: f_{1}(x)=1} f_{2}(x)$ and $c_{2}=-\max _{x: f_{1}(x)=1} f_{2}(x)$.

Proof. Define the set $E=\left\{x: f_{i}(x)=1\right\}$. Since $f_{1}(x)$ is continuous, the set $E$ is closed. Furthermore, it can shown that since $f_{1}$ is continuous, homogenous and positive-definite, $f_{1}$ is radially unbounded, and therefore $E$ is a bounded set. Consequently, $E$ is compact. Hence, $c_{1}$ and $c_{2}$ are well-defined, and hence by definition,

$$
-c_{1} \leq f_{2}(x) \leq-c_{2}
$$

for all $x \in E$. Now, consider any $x \neq 0$. Then, setting $y=\delta_{f_{1}^{-1 / k_{1}}(x)}^{r}(x)$, we have by homogeneity that

$$
\begin{aligned}
f_{1}(y) & =f_{1}\left(\delta_{f_{1}^{-1 / k_{1}}(x)}^{r}(x)\right), \\
& \left.=\left(f_{1}^{-1 / k_{1}}(x)\right)^{k_{1}} f_{1}(x)\right), \\
& =1 .
\end{aligned}
$$

Hence, $y \in E$, and by (2.9),

$$
-c_{1} \leq f_{2}(y)=f_{2}\left(\delta_{f_{1}^{-1 / k_{1}}(x)}^{r}(x)\right) \leq-c_{2}
$$

By homogeneity $\left.f_{2}\left(\delta_{f_{1}^{-1 / k_{1}}(x)}^{r}(x)\right)=\left(f_{1}^{-1 / k_{1}}(x)\right)^{k_{2}} f_{2}(x)\right)=f_{1}^{-k_{2} / k_{1}}(x) f_{2}(x)$. Substituting this into (2.10) and rearranging yields (2.8) for all $x \in \mathbb{R} \backslash\{0\}$. Finally, (2.8) holds trivially for $x=0$, by Proposition 1 this concludes the proof.

Proposition 6. (Rosier) [Rosier, 1992].

Consider the map $\phi:(0, \infty) \times S^{n-1} \mapsto \mathbb{R}^{n} \backslash\{0\}$ (where $S^{n-1}$ denotes the unit sphere in $\left.\mathbb{R}^{n}\right)$, defined by $\phi(t, x)=\delta_{t}^{r}(x)$. Then, $\phi$ is a bijection. Furthermore, denoting its inverse $\phi^{-1}: \mathbb{R}^{n} \backslash\{0\} \mapsto(0, \infty) \times S^{n-1}$, by

$$
\psi(x)=\phi^{-1}(x)=\left(\psi_{t}(x), \psi_{y}(x)\right)
$$


Then, $\psi_{t}, \psi_{y}$ are $\mathcal{C}^{\infty}$ on $\mathbb{R}^{n} \backslash\{0\}$, and

$$
\lim _{\|x\| \rightarrow 0} \psi_{t}(x)=0, \quad \lim _{\|x\| \rightarrow \infty} \psi_{t}(x)=\infty
$$

\subsection{Lyapunov Stability of Systems}

Consider the system described by

$$
\dot{\mathbf{x}}=f(\mathbf{x}, t) .
$$

Definition 2.1. (Lyapunov stability) The equilibrium point $\mathbf{x}_{e}=0$ of (2.11) is said to be

1. stable if, for every $\epsilon>0$, there exist a $\delta=\delta(\epsilon)>0$ such that

$$
\|\mathbf{x}(0)\|<0 \Rightarrow\|\mathbf{x}(t)\|<\epsilon \quad \forall t \geq 0,
$$

2. unstable if it is not stable,

3. asymptotically stable if it is stable and $\delta$ can be chosen such that

$$
\|\mathbf{x}(0)\|<\delta \Rightarrow \lim _{t \rightarrow \infty} \mathbf{x}(t)=0
$$

Definition 2.4.1. Exponential stability.

The equilibrium point $\mathbf{x}_{e}=0$ of (2.11) is exponentially stable if there exist positive constants $\delta, k$, and $\lambda$ such that

$$
\|\mathbf{x}(t)\| \leq k\|\mathbf{x}(0)\| e^{-\lambda} t, \quad \forall\|\mathbf{x}(0)\| \leq \delta \quad \forall t \geq 0
$$

Given the definitions, we can now state the Lyapunov theorem for stability.

Theorem 2.4 (Lyapunov theorem). Let $V: D \rightarrow \mathbb{R}$ be a continuous differential function such that

$$
V(0)=0 \text { and } V(\mathbf{x})>0 \text { in } D \backslash\{0\}
$$

The equilibrium point $\mathbf{x}_{e}$ is said to be 
1. stable if

$$
\dot{V}(\mathbf{x}) \leq 0 \quad \forall \mathbf{x} \in D
$$

2. asymptotically stable if

$$
\dot{V}(\mathbf{x}) \leq 0 \quad \forall \mathbf{x} \in D \backslash\{0\}
$$

The above definitions and theorem are valid in a neighborhood $D$ around an equilibrium point. We define the theorem for global stability next.

Theorem 2.5. Let $V: \mathbb{R}^{n} \rightarrow \mathbb{R}$ be a continuous differential function such that

$$
V(0)=0 \quad \text { and } \quad V(\mathbf{x})>0 \quad \forall \mathbf{x} \neq 0 .
$$

If $V(\mathbf{x})$ is radially unbounded $(\|\mathbf{x}\| \rightarrow \infty \Rightarrow V(\mathbf{x}) \rightarrow \infty)$ and

$$
\dot{V}(\mathbf{x})<0 \quad \forall \mathbf{x} \neq 0
$$

then the equilibrium point $\mathbf{x}_{e}=0$ is said to be globally asymptotically stable.

Definition 2.4.2. Invariant set.

A set $S$ in the phase space is said to be invariant with respect to (2.11) if

$$
\mathbf{x}(0) \in S \Rightarrow \mathbf{x}(t) \in S \quad \forall t \in \mathbb{R}
$$

Definition 2.4.3. Positively invariant set.

A set $S$ in the phase space is said to be positively invariant with respect to (2.11) if

$$
\mathbf{x}(0) \in S \Rightarrow \mathbf{x}(t) \in S \quad \forall t \geq 0 .
$$

We can now state La Salle's theorem.

Theorem 2.6 (La Salle's theorem). Let $\Omega \subset D$ be a compact set that is positively invariant with respect to (2.11). Let $V: D \rightarrow \mathbb{R}$ be a continuously differentiable function such that $\dot{V}(\mathbf{x}) \leq 0$ in $\Omega$. Let $E$ be the set of all points in $\Omega$ where $\dot{V}(\mathbf{x})=0$. Let $M$ be the largest invariant set in $E$. Then every solution starting in $\Omega$ approaches $M$ as $t \rightarrow \infty$. 


\subsubsection{Finite-time stability}

Consider the system

$$
\dot{x}=f(x, t), f(0, t)=0 \quad \forall t \geq 0,
$$

where $f: U \times \mathbb{R}_{+} \rightarrow \mathbb{R}$ is continuous on an open neighbourhood $U$ of the origin $x=0$. The origin of the system is (locally) finite-time stable if it is Lyapunov stable and finite-time convergent in a neighborhood $U_{0} \subset U$ of the origin. Finite-time convergence means that for any non-zero initial condition $x\left(t_{0}\right)=x_{0} \in U_{0}$ at any initial time $t_{0} \in \mathbb{R}^{+}$, there exists a settling time $T\left(x_{0}, t_{0}\right)>0$ such that every solution $x\left(t ; t_{0}, x_{0}\right)$ is defined for $\left[t_{0}, T\right), x\left(t ; t_{0}, x_{0}\right) \in U_{0} \backslash\{0\}$ for $t_{0} \leq t \leq T, x\left(t ; t_{0}, x_{0}\right)=0$ for $t>T$ and $\lim _{t \rightarrow T} x\left(t ; t_{0}, x_{0}\right)=0$. When $U=U_{0}=\mathbb{R}^{n}$, the origin of the system is said to be globally finite-time stable. Next we illustrate some Lyapunov finite-time stability results.

Lemma 2.7. Suppose that there is a continuously differentiable positive-definite function $V(x)$ on a neighborhood $U_{1} \subset U$ of the origin, such that

$$
L_{f} V(x) \leq-k V^{\beta}(x), \forall x \in U_{1}, \forall t \in \mathbb{R}^{+},
$$

where $k>0$ and $0<\beta<1$, and $L_{f} V(x)$ is the Lie derivative of $V$ along the trajectory $f(x, t)$. then the origin of the system (2.22) is locally finite-time stable. The settling time satisfies

$$
T\left(x\left(t_{0}\right), t_{0}\right) \leq t_{0}+V^{1-\beta}\left(x\left(t_{0}\right)\right) /(k(1-\beta)) .
$$

Proof. The condition (2.23) implies that the origin is asymptotically stable (since $\dot{V}=L_{f} V(x)<0$ in a neighborhood of the origin, and $V(x)$ does not depend on $\mathrm{t}$ (otherwise additional conditions on $V$ might need to be considered)). Consequently, there exists a neighborhood $U_{0} \subset U_{1}$ of the origin that $x\left(t_{0}\right) \in U_{0}$ implies that $x\left(t ; t_{0}, x_{0}\right) \in U_{0}$ for all $t \geq t_{0}$ and $\lim _{t \rightarrow \infty} x\left(t ; t_{0}, x_{0}\right)=0$. Now, consider a trajectory such that of (2.22) and along this trajectory, using (2.23), we have

$$
\dot{V}(x(t)) \leq-k V^{\beta}(x(t))
$$

for all $t>t_{0}$. Suppose that $x\left(t ; t_{0}, x_{0}\right) \neq 0$ on some interval $t \in\left[t_{0}, \bar{t}\right)$ for some $\bar{t}>t_{0}$. 
Then, define a function $W(x)=V^{1-\beta}(x)$. Since this is continuously differentiable on $U_{1} \backslash\{0\}$, we have for $t \in\left[t_{0}, \bar{t}\right)$

$$
\dot{W}(x(t))=(1-\beta) V^{-\beta}(x(t)) \dot{V}(x(t)) \leq-k(1-\beta) V^{-\beta}(x(t)) V^{\beta}(x(t)),
$$

which leads to

$$
\dot{W}(x(t)) \leq-k(1-\beta) .
$$

Integrating this from $t_{0}$ to $t$, we obtain the following:

$$
W(x(t)) \leq W\left(x\left(t_{0}\right)\right)-k(1-\beta)\left(t-t_{0}\right)
$$

Hence, $W(x(t))$ and $V(x(t))$ must converge to zero in finite-time and this occurs when the right-hand side of (2.25) becomes zero, which happens when

$$
V\left(x\left(t_{0}\right)\right)^{1-\beta}=W\left(x\left(t_{0}\right)\right)=k(1-\beta) t-k(1-\beta) t_{0}
$$

which can be rearranged to give the right-hand side of (2.24). This concludes the proof.

\subsection{Optimal Control}

The general notion of the optimal control problem is to search for a control sequence or history of the control vector $u(t) \in U$ for $t_{0} \leq t \leq t_{f}$, in which forces the system state from initial value to its final value along a trajectory and minimizes (or maximizes) a cost function, $J(q(t), u(t), t)$. The state history $q(t) \in M$ for $t_{0} \leq t \leq t_{f}$ in which a result from the application of the (optimal) control history is called an optimal trajectory.

Consider the control system of the form:

$$
\dot{q}(t)=f_{u}(q(t), u(t), t), \quad q(t) \in M, \quad u(t) \in U \subset \mathbb{R}^{m}
$$

where $M$ is a class $C^{\infty}$-manifold, $U$ is an arbitrary subset of $\mathbb{R}^{m}$ called the control set and $f_{u}(q(t), u(t), t)$ is a smooth vector field on $M$ for a given $u(t) \in U$. Moreover, the 
admissible controls $u(t) \in U$ are locally bounded mappings. Next, we substitute the control $u(t)$ into the control system (2.26) to obtain a ordinary differential equation $\dot{q}(t)=f_{u}(q(t), u(t), t)$. By Carathéodory's Theorem, any point $q_{0} \in M$ the system can be identified as a Cauchy problem [Agrachev and Sachkov, 2004]

$$
\dot{q}(t)=f_{u}(q(t), u(t), t) \quad q\left(t_{0}\right)=q_{0}
$$

which has a unique solution. In order to find the optimal control, we need to compare all the admissible controls attainable on the interval $\left[t_{0}, t_{f}\right]$ by using the following cost functional:

$$
J(q(t), u(t), t)=\phi\left[q\left(t_{f}\right), t_{f}\right]+\int_{t_{0}}^{t_{f}} \mathcal{L}\left[q_{u}(t), u(t), t\right] d t
$$

where $\phi: M \times R \rightarrow R$ is the end cost function, the integrand $\mathcal{L}: M \times U \times \mathbb{R} \rightarrow \mathbb{R}$ (also known as the Lagrangian) is a continuous mapping, $q_{u}(t)$ is a corresponding solution (optimal trajectory) to problem (2.28) and $u(t)$ is the control history on interval $\left[t_{0}, t_{f}\right]$.

\subsubsection{The optimal control problem}

Given all admissible control $u=u(t)$ in $t \in\left[t_{0}, t_{f}\right]$, find the corresponding solution $q_{u}(t)$ of the Cauchy problem that minimizes the cost functional $J(q(t), u(t), t)$ and satisfying the boundary condition:

$$
\operatorname{minimize} \quad J\left(q(t), u(t), t_{0}, t_{f}\right)=\phi\left[q_{f}, t_{f}\right]+\int_{t_{0}}^{t_{f}} \mathcal{L}\left[q_{u}(t), u(t), t\right] d t
$$

such that

$$
\begin{aligned}
\dot{q}(t) & =f_{u}(q(t), u(t), t) \\
e^{L} & \leq e\left(q_{0}, q_{f}, t_{0}, t_{f}\right) \leq e^{U} \\
h^{L} & \leq h(q(t), u(t), t) \leq h^{U}
\end{aligned}
$$

where $q_{0} \equiv q\left(t_{0}\right), q_{f} \equiv q\left(t_{f}\right), e^{L}, e^{U} \in \mathbb{R}^{N_{e}}$ are the lower and upper bounds on the endpoint function, $e: M \times M \times \mathbb{R} \times \mathbb{R} \rightarrow \mathbb{R}^{N_{e}}, h^{L}, h^{U} \in \mathbb{R}^{N_{h}}$ are the lower and 
upper bounds on the constraints function, $h: M \times U \times \mathbb{R} \rightarrow \mathbb{R}^{N_{h}}$. It is assumed that all nonlinear functions in problem (2.29) are continuously differentiable and Lipschitz-continuous over the domain $M \times U \times \mathbb{R} \times \mathbb{R}$. The optimal control problem is the minimization problem for $J\left(q(t), u(t), t_{0}, t_{f}\right)$ with control constraints and fixed boundary conditions. 


\section{CHAPTER 3}

\section{Velocity-Free Nadir-pointing}

\section{Attitude Stabilization}

This chapter examines the attitude stabilization of an underactuated nadir-pointing rigid spacecraft in a circular orbit in the presence of time-varying disturbances without any angular velocity measurements (velocity-free). The aim of this chapter is to develop a control system capable of stabilizing the underactuated rigid-body spacecraft system relative to its orbit. This is particularly important to satellites; for example, to ensure optimal positioning antenna for stable communication or the pointing of Earth-observing payloads. The spacecraft is placed in an orbital frame and the goal is to stabilize the spacecraft relative to this frame. Aside from spacecraft control we investigate a case where the angular velocity measurements is not available. A nonlinear observer is used to estimate the angular velocity of the spacecraft to complete an output feedback control law. A sliding mode control strategy is proposed for this stabilization problem in conjunction with a nonlinear observer. The Local ultimate-boundedness of the closed-loop system is rigorously proven. Numerical examples demonstrate the performance of the controller in the presence of timevarying disturbances, non-zero orbital eccentricity, inertia matrix uncertainties and measurement noise in a sampled-data implementation. In particular, even though the theoretical ultimate-boundedness guarantees are local, the numerical examples demonstrate ultimate-boundedness even with large initial attitude errors. 


\subsection{Introduction}

Angular velocity is a vital information that is required by most control strategies, measured using sensors or computed numerically using various parameterizations [Hughes, 1986]. There are works in literature investigating the underactuated spacecraft, some relevant results are outlined here.

Authors in [Gui, Vukovich, and S. Xu, 2015] successfully treated the control of underactuated rigid spacecraft with two internal control torques by using a transverse function method and recently, in [Gui and Vukovich, 2017], a finite-time angular velocity observer was proposed. While the majority of the existing research in the literature on spacecraft attitude control use full-state feedback (attitude and angular velocity measurements are available) for control law formulation as such in the works by [G. Godard and K. Kumar, 2010; Godard, K. Kumar, and A. Zou, 2013; G. Godard, 2010; Trumpf et al., 2012; Khosravian and Namvar, 2012; Khosravian and Namvar, 2010; Tayebi, 2007; Seo and Akella, 2007], there has also been interest in velocity-free attitude control, since the availability of angular velocity measurements is not always satisfied for reasons such as cost limitations or rate gyro failure. The use of nonlinear observers were first discussed by [Salcudean, 1991], where the motivation was to obtain the angular velocity of a rigid-body from orientation and torque measurements only, without the noisy numerical differentiation. Examples of velocity-free control include [Lizarralde and Wen, 1996], where the authors developed a nonlinear filter of the quaternion using passivity approach without directly utilizing angular velocity measurements for a robot control problem. [Thakur and Akella, 2015] utilized velocity-free attitude-stabilization control law that ensures stabilization to the prescribed constant orientation using solely on vector measurements for feedback. Chunodkar and Akella [Chunodkar and Akella, 2014] developed an exponentially converging switching velocity observer for rigid-body attitude tracking control without angular velocity measurements and is independent of controller design and proved almost global exponential stability for the estimation error dynamics. The switching framework enabled the addition of terms to ensure Lyapunov stability. Recent works regarding velocity-free attitude control problem is published by [Xiao et al., 2014] and [Zlotnik and J. R. Forbes, 2017], where the 
authors in [Xiao et al., 2014] developed a velocity-measurement free control scheme in conjunction with a nonlinear observer for fault-tolerant control system of a fully actuated spacecraft with actuator torque bias and in the presence of external disturbances. [Zlotnik and J. R. Forbes, 2017] developed nonlinear attitude estimator based on direct vector measurements while utilizing the attitude error function to achieve desirable convergence properties.

To the author's best knowledge, velocity-free underactuated spacecraft attitude control has not been dealt with in the existing literature. The majority of the aforementioned literature on underactuated spacecraft attitude control is for stabilization to an inertially fixed desired attitude. By contrast, this chapter considers the underactuated attitude stabilization to a nadir-pointing attitude, similar to [Godard, K. Kumar, and A. Zou, 2013; G. Godard, 2010; G. Godard and K.D. Kumar, 2011] which outlined strategies involving the control of different underactuated axes. Notable improvements in this chapter over Godard et al. [Godard, K. Kumar, and A. Zou, 2013; G. Godard, 2010] include the following: 1) the control law in this chapter does not require angular velocity measurements; 2) the control law in this chapter does not require knowledge of the disturbance torques (needed in the computation of the higher order sliding mode in Godard et al. [Godard, K. Kumar, and A. Zou, 2013; G. Godard, 2010]); 3) this chapter considers the full nonlinear dynamics in the analysis of the closed-loop system behavior within a boundary layer of the sliding mode; 4) The full-state feedback in [G. Godard and K.D. Kumar, 2011] utilized higher order sliding mode which required a priori knowledge of the disturbances that needed to be met, this is not the case for the full-state feedback control law developed here.

Chapter 3 is organized in the following manner. Section 3.2, presents the problem formulation including the development of the relative underactuated rigid-body spacecraft attitude dynamics and the control objective needed to be solved. In section 3.3 homogeneity of functions is used to design the observer-based control law, and the stability analysis is rigorously presented. Several examples of umerical simulations demonstrating the observer-based control law performance are included in Section 3.5. Finally, Section 3.6 summarizes the chapter. 


\subsection{Problem Formulation}

In this section the coordinate system used, the kinematics and dynamics of the spacecraft model are outlined, and the attitude control problem is defined.

\subsubsection{Coordinate frames}

As seen in Figure 3.1, the Earth-centered inertial frame, denoted by $\mathfrak{F}_{I}$, has its origin at the center of the Earth, with the Z-axis passing through the celestial north pole, the $X$-axis directed towards the vernal equinox, and the $Y$-axis completes the righthanded triad. The orbital frame, as denoted by $\mathfrak{F}_{n}$, follows the instantaneous flight path with origin at the spacecraft's center of mass with the $z_{n}$-axis pointing in the nadir direction, the $y_{n}$-axis points along the direction of the orbit anti-normal and the $x_{n}$-axis completing the triad. The spacecraft body-fixed frame is denoted by $\mathfrak{F}_{b}$.

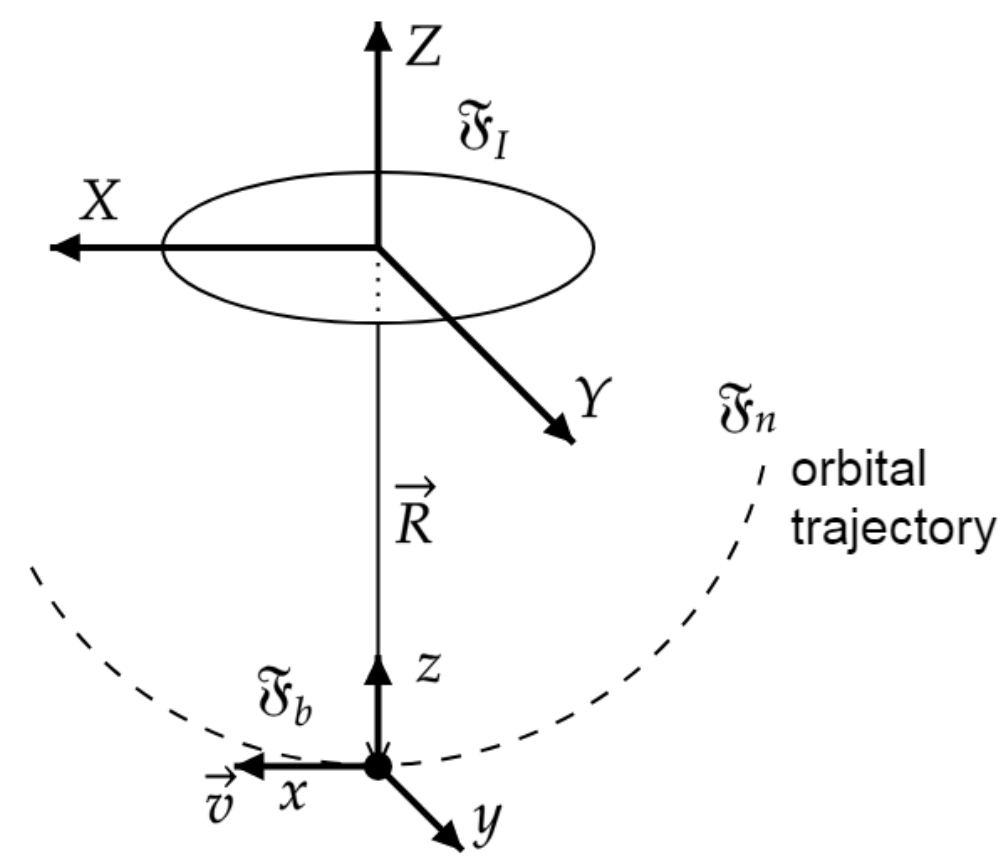

FIGURE 3.1: Coordinate Topologies 


\subsubsection{Spacecraft attitude kinematics and dynamics}

The spacecraft is assumed to be in a Keplerian circular orbit of radius $R$. The corresponding orbital angular velocity is $\omega_{0}=\sqrt{\mu / R^{3}}$, where $\mu \in \mathbb{R}$ represents the gravitational parameter of the Earth [Ruiter, Damaren, and J. Forbes, 2013].

The orientation of the body-fixed frame relative to the orbital frame is represented by a unit quaternion $\left[\epsilon^{T} \eta\right]^{T} \in \mathbb{S}^{3}$, which can be written as

$$
\left[\begin{array}{l}
\epsilon \\
\eta
\end{array}\right]=\left[\begin{array}{c}
e \sin (\phi / 2) \\
\cos (\phi / 2)
\end{array}\right],
$$

where $e \in \mathrm{S}^{2}$ and $\phi \in[-\pi, \pi]$ are the corresponding principal axis and angle of rotation, respectively. Note that $\epsilon \in \mathbb{R}^{3}$ is the vector part of the quaternion, while $\eta \in \mathbb{R}$ is the scalar part.

The corresponding transformation matrix $C_{b n} \in S O(3)$ that describes the rotational transformation from orbital coordinates to body coordinates is given by

$$
C_{b n}=\left(\eta^{2}-\epsilon^{T} \epsilon\right) I+2 \epsilon \epsilon^{T}-2 \eta \epsilon^{\times},
$$

where $\epsilon^{\times} \in \mathbb{R}^{3 \times 3}$ denotes the skew-symmetric matrix of the vector $\epsilon$ as given by

$$
\epsilon^{\times}=\left[\begin{array}{ccc}
0 & -\epsilon_{3} & \epsilon_{2} \\
\epsilon_{3} & 0 & -\epsilon_{1} \\
-\epsilon_{2} & \epsilon_{1} & 0
\end{array}\right] .
$$

With the defined orbiting frame $\mathfrak{F}_{n}$, the angular velocity of spacecraft relative to the inertial frame, $\omega_{b I} \in \mathbb{R}^{3}$, given in the body-fixed frame is given by,

$$
\omega_{b I}=\omega_{b n}-\omega_{0} C_{b n} e_{2}
$$

where, $\omega_{b n} \in \mathbb{R}^{3}$ is the relative body-orbital angular velocity. 
The kinematics equation of the spacecraft relative to the orbiting frame $\mathfrak{F}_{n}$ are given by,

$$
\left[\begin{array}{c}
\dot{\epsilon} \\
\dot{\eta}
\end{array}\right]=\frac{1}{2}\left[\begin{array}{c}
\eta I+\epsilon^{\times} \\
-\epsilon^{T}
\end{array}\right] \omega_{b n} .
$$

The spacecraft dynamics are governed by Euler's equation given by [Hughes, 1986]

$$
J \dot{\omega}_{b I}+\omega_{b I}^{\times} J \omega_{b I}=\tau_{u}+\tau_{g}+\tau_{d}
$$

where $J \in \mathbb{R}^{3 \times 3}$ is the spacecraft inertia matrix, $\tau_{u}$ is the control torque, $\tau_{g}$ is the gravity-gradient torque and $\tau_{d}$ are the all other disturbance torques acting on the spacecraft. The spacecraft inertia matrix is assumed to have the form

$$
J=\operatorname{diag}\left\{J_{x}, J_{y}, J_{z}\right\}
$$

It is assumed that the spacecraft is only actuated about the pitch and yaw axes, such that

$$
\tau_{u}=\left[0, \tau_{y}, \tau_{z}\right]^{T}
$$

Remark. If the underactuated axis is the pitch axis, then due to the nature of the attitude dynamics of the spacecraft, three-axis stability is not achievable directly.

The torque due to the gravity gradient, is given by [Hughes, 1986]

$$
\tau_{g}=3 \omega_{0}^{2}\left(C_{b n} e_{3}\right)^{\times} J C_{b n} e_{3}
$$

\subsubsection{Control objective}

The control objective in this chapter is to regulate the body-orbital states $\left(\epsilon, \omega_{b n}\right)$ to a neighborhood of the origin, without the use of angular velocity measurements. Due to the assumption of bounded time-varying disturbances, there is no stable equilibrium for which the control can drive to and due nature of underactuated system, it is more realistic to state that control objective be in the neighbourhood of the origin. 
Substitution of (3.4) and (3.8) into (3.6), together with (3.5) lead to the equations of motion for $\epsilon$ and $\omega$, which are

$$
\begin{aligned}
\dot{\epsilon}= & \frac{1}{2}\left(\eta I+\epsilon^{\times}\right) \omega_{b n}, \\
\dot{\omega}_{b n}= & J^{-1}\left(-\left(\omega_{b n}-\omega_{0} C_{b n} e_{2}\right)^{\times} J\left(\omega_{b n}-\omega_{0} C_{b n} e_{2}\right)\right. \\
& \left.+3 \omega_{0}^{2}\left(C_{b n} e_{3}\right)^{\times} J C_{b n} e_{3}-\omega_{0} J \omega_{b n}^{\times} C_{b n} e_{2}+\tau_{u}+\tau_{d}\right),
\end{aligned}
$$

\subsection{Design of Underactuated Attitude Control Law}

Since a local stability result will be obtained in this chapter, it is assumed that $\eta(t)>$ 0 for all $t \geq 0$ (the attitude error does not reach 180 degrees in rotation). Since $(\epsilon, \eta)$ and $(-\epsilon,-\eta)$ represent the same attitude, one can take (choose the representation $(\epsilon, \eta)$ for which $\eta>0)$

$$
\eta=\sqrt{1-\epsilon^{T} \epsilon}
$$

The kinematics in (3.9) can therefore be written as

$$
\dot{\epsilon}=\frac{1}{2} \omega+\frac{1}{2}(A(\epsilon)-I) \omega
$$

where

$$
A(\epsilon)=\sqrt{1-\epsilon^{T} \epsilon} I+\epsilon^{\times}
$$

The following well-known fact will be useful:

$$
\|A(\epsilon)\|=1
$$

for all $\epsilon$ satisfying $\|\epsilon\| \leq 1$. Using (3.14), the following result can be obtained.

Proposition 7. $\|A(\epsilon)-I\| \leq\|\epsilon\|(1+\|\epsilon\|)$, for all $\epsilon$ satisfying $\|\epsilon\| \leq 1$.

Proof. Consider the function $f(a)=\|\sqrt{(1-a)}-1\|=1-\sqrt{1-a}$ for $0 \leq a \leq 1$, and define $g(a)=a-f(a)$. The derivative of $g(a)$ for $0 \leq a \leq 1$ is given by

$$
\frac{d g}{d a}=1-\frac{1}{2 \sqrt{1-a}}
$$


Hence, at $a=0$ we have $d g / d a=1 / 2$. This means that $g(a)$ is initially increasing. Hence since $g(0)=0$, we have $g(a)>0$ for $0 \leq a \leq \alpha$, for some $1 \geq a \geq 0$. Now, suppose that at some point $1 \geq a>\alpha, g(a)<0$. By the intermediate value theorem (since $g$ is continuous), there exist $\alpha<\bar{a}<a$, such that $g(\bar{a})=0$. This point satisfies $f(\bar{a})=\bar{a}$. Expanding and re-arranging gives $1-\bar{a}=\sqrt{1-\bar{a}}$, which implies that $(1-\bar{a})^{2}=1-\bar{a}$, which leads to $\bar{a}(1-\bar{a})=0$. This has only two possibilities, $\bar{a}=0,1$. Hence, we have $g(a) \geq 0$ for all $0 \leq a \leq 1$, which implies that $f(a) \leq a$ for all $0 \leq a \leq 1$. Consequently, taking $a=\epsilon^{T} \epsilon$, we have

$$
\left\|\sqrt{1-\epsilon^{T} \epsilon}-1\right\| \leq\|\epsilon\|^{2}
$$

for all $\epsilon$ satisfying $\|\epsilon\| \leq 1$. Finally,

$$
\begin{aligned}
\|A(\epsilon)-I\| & =\left\|\left(\sqrt{1-\epsilon^{T} \epsilon}-1\right) I+\epsilon^{\times}\right\|, \\
& \leq\left\|\left(\sqrt{1-\epsilon^{T} \epsilon}-1\right) I\right\|+\left\|\epsilon^{\times}\right\|, \\
& \leq\|\epsilon\|^{2}+\|\epsilon\|,
\end{aligned}
$$

where $\left\|\epsilon^{\times}\right\|=\|\epsilon\|$ has been made used of. This concludes the proof.

Proposition 7 shows that the kinematics in (3.12) consist of a linear part and a second order part in $\left(\epsilon, \omega_{b n}\right)$. A similar expansion for the dynamics equation in (3.10) is now obtained. First, rewrite (3.10) as

$$
\begin{aligned}
\dot{\omega}_{b n}= & J^{-1}\left(-\omega_{b n}^{\times} J \omega_{b n}+\omega_{0}\left(\omega_{b n}^{\times} J-J \omega_{b n}^{\times}-\left(J \omega_{b n}\right)^{\times}\right) C_{b n} e_{2}\right. \\
& \left.+3 \omega_{0}^{2}\left(C_{b n} e_{3}\right)^{\times} J C_{b n} e_{3}-\omega_{0}^{2}\left(C_{b n} e_{2}\right)^{\times} J C_{b n} e_{2}+\tau_{u}+\tau_{d}\right) .
\end{aligned}
$$

Each of the terms on the right hand side of (3.15) is now expanded. It is essential to separate the linear and nonlinear terms of the equations of motion. This will allows each term to be analyzed and design a control system to drive these terms to an 
ultimately bound. First, we have

$$
-J^{-1} \omega_{b n}^{\times} J \omega_{b n}=\left[\begin{array}{c}
\frac{J_{y}-J_{z}}{J_{x}} \omega_{y} \omega_{z} \\
\frac{J_{z}-J_{x}}{J_{y}} \omega_{x} \omega_{z} \\
\frac{J_{x}-J_{y}}{J_{z}} \omega_{x} \omega_{y}
\end{array}\right]
$$

which is second order in $\omega$. Next, using the identity $\epsilon^{\times} \epsilon^{\times}=\epsilon \epsilon^{T}-\epsilon^{T} \epsilon I$ [Hughes, 1986] together with (3.11) and the definition in (3.13), we get

$$
\begin{aligned}
C_{b n} & =\left(\eta^{2}-\epsilon^{T} \epsilon\right) I+2 \epsilon^{T} \epsilon-2 \eta \epsilon^{\times}, \\
& =\left(1-\epsilon^{T} \epsilon\right) I+2 \epsilon^{T} \epsilon-2 \eta \epsilon^{\times}, \\
& =I+2 \epsilon^{\times} \epsilon^{\times}-2 \eta \epsilon^{\times}, \\
& =I+2\left(\eta I-\epsilon^{\times}\right) \epsilon^{\times}, \\
& =I-2 A^{T}(\epsilon) \epsilon^{\times} .
\end{aligned}
$$

Consequently, $C_{b n} e_{2}=e_{2}-2 A^{T}(\epsilon) \epsilon^{\times}$, and upon expansion the second term in (3.15) becomes

$$
\begin{aligned}
\omega_{0} J^{-1}\left(\omega_{b n}^{\times} J-J \omega_{b n}^{\times}-\left(J \omega_{b n}\right)^{\times}\right) C_{b n} e_{2}= & {\left[\begin{array}{c}
\frac{J_{x}+J_{z}-J_{y}}{J_{x}} \omega_{0} \omega_{z} \\
0 \\
-\frac{J_{x}+J_{z}-J_{y}}{J_{z}} \omega_{0} \omega_{x}
\end{array}\right] } \\
& +2 \omega_{0} J^{-1}\left(\left(J A^{T}(\epsilon) \epsilon^{\times} e_{2}\right)^{\times}\right. \\
& \left.-J\left(A^{T}(\epsilon) \epsilon^{\times} e_{2}\right)^{\times}-\left(A^{T}(\epsilon) \epsilon^{\times} e_{2}\right)^{\times} J\right) \omega_{b n},
\end{aligned}
$$

which consists of a linear and second order part in $\left(\epsilon, \omega_{b n}\right)$. For the third term in (3.15), first expand

$$
\begin{aligned}
\left(C_{b n} e_{3}\right)^{\times} J C_{b n} e_{3} & =\left(e_{3}-2 A^{T}(\epsilon) \epsilon^{\times} e_{3}\right)^{\times} J\left(e_{3}-2 A^{T}(\epsilon) \epsilon^{\times} e_{3}\right), \\
& =e_{3}^{\times} J e_{3}+2\left(\left(J e_{3}\right)^{\times}-e_{3}^{\times} J\right) A^{T}(\epsilon) \epsilon^{\times} e_{3}+4\left(A^{T}(\epsilon) \epsilon^{\times} e_{3}\right)^{\times} J A^{T}(\epsilon) \epsilon^{\times} e_{3} .
\end{aligned}
$$


Since $J$ is diagonal and $e_{3}=[0,0,1]^{T}$, therefore $e_{3}^{\times} J e_{3}=0$. Next, using $A^{T}(\epsilon)=$ $I+A^{T}(\epsilon)-I$, we have

$2\left(\left(J e_{3}\right)^{\times}-e_{3}^{\times} J\right) A^{T}(\epsilon) \epsilon^{\times} e_{3}=2\left(\left(J e_{3}\right)^{\times}-e_{3}^{\times} J\right) \epsilon^{\times} e_{3}+2\left(\left(J e_{3}\right)^{\times}-e_{3}^{\times} J\right)\left(A^{T}(\epsilon)-I\right) \epsilon^{\times} e_{3}$,

and expanding the first term leads to

$2\left(\left(J e_{3}\right)^{\times}-e_{3}^{\times} J\right) A^{T}(\epsilon) \epsilon^{\times} e_{3}=2\left[\begin{array}{c}\left(J_{z}-J_{y}\right) \epsilon_{1} \\ \left(J_{z}-J_{x}\right) \epsilon_{2} \\ 0\end{array}\right]+2\left(\left(J e_{3}\right)^{\times}-e_{3}^{\times} J\right)\left(A^{T}(\epsilon)-I\right) \epsilon^{\times} e_{3}$.

Making use of the above results, one obtains

$$
\begin{aligned}
3 \omega_{0}^{2} J^{-1}\left(C_{b n} e_{3}\right)^{\times} J C_{b n} e_{3}= & 6 \omega_{0}^{2}\left[\begin{array}{c}
\frac{J_{z}-J_{y}}{J_{x}} \epsilon_{1} \\
\frac{J_{z}-J_{x}}{J_{y}} \epsilon_{2} \\
0
\end{array}\right]+6 \omega_{0}^{2}\left(\left(J e_{3}\right)^{\times}-e_{3}^{\times} J\right)\left(A^{T}(\epsilon)-I\right) \epsilon^{\times} e_{3} \\
& +12 \omega_{0}^{2}\left(A^{T}(\epsilon) \epsilon^{\times} e_{3}\right)^{\times} J A^{T}(\epsilon) \epsilon^{\times} e_{3}
\end{aligned}
$$

which consists of a linear and second order part in $\left(\epsilon, \omega_{b n}\right)\left(A^{T}(\epsilon)-I\right.$ is first order in $\epsilon$, and this is multiplied by $\epsilon^{\times}$in (3.19) making the term second order). In a similar manner, one obtains

$$
\begin{aligned}
-\omega_{0}^{2} J^{-1}\left(C_{b n} e_{2}\right)^{\times} J C_{b n} e_{2}= & 2 \omega_{0}^{2}\left[\begin{array}{c}
\frac{J_{z}-J_{y}}{J_{x}} \epsilon_{1} \\
0 \\
\\
\frac{J_{x}-J_{y}}{J_{z}} \epsilon_{3}
\end{array}\right]-2 \omega_{0}^{2}\left(\left(J e_{2}\right)^{\times}-e_{2}^{\times} J\right)\left(A^{T}(\epsilon)-I\right) \epsilon^{\times} e_{2} \\
& -4 \omega_{0}^{2}\left(A^{T}(\epsilon) \epsilon^{\times} e_{2}\right)^{\times} J A^{T}(\epsilon) \epsilon^{\times} e_{2}
\end{aligned}
$$

which also consists of a linear and second order part in $\left(\epsilon, \omega_{b n}\right)$.

\subsubsection{Control design for unactuated states}

Defining the state vector $x=\left[\begin{array}{ll}\epsilon^{T} & \omega_{b n}^{T}\end{array}\right]^{T}$, it is now partitioned into actuated and unactuated states by defining $x_{1}=\left[\begin{array}{ll}\epsilon^{T} & \omega_{x}\end{array}\right]^{T}$, and $x_{2}=\left[\omega_{y}, \omega_{z}\right]^{T}$. Using the above 
developments, and noting that $\omega_{b n}=\omega_{x} e_{1}+\left[e_{2} e_{3}\right] x_{2}$, one can now obtain the dynamics for the unactuated states as

$$
\dot{x}_{1}=\bar{A}_{11} x_{1}+\bar{A}_{12} x_{2}+g\left(x_{1}\right)+h\left(x_{1}, x_{2}\right)+d,
$$

where

$$
\begin{aligned}
& \bar{A}_{11}=\left[\begin{array}{cccc}
0 & 0 & 0 & 1 / 2 \\
0 & 0 & 0 & 0 \\
0 & 0 & 0 & 0 \\
8 \omega_{0}^{2}\left(J_{z}-J_{y}\right) / J_{x} & 0 & 0 & 0
\end{array}\right], \bar{A}_{12}=\left[\begin{array}{cc}
0 & 0 \\
1 / 2 & 0 \\
0 & 1 / 2 \\
0 & \omega_{0}\left(J_{x}+J_{z}-J_{y}\right) / J_{x}
\end{array}\right], \\
& g\left(x_{1}\right)=\left[\begin{array}{c}
\omega_{x}(A(\epsilon)-I) e_{1} / 2 \\
a\left(x_{1}\right),
\end{array}\right] \\
& a\left(x_{1}\right)=2 \omega_{0} \omega_{x} e_{1}^{T} J^{-1}\left(\left(J A^{T}(\epsilon) \epsilon^{\times} e_{2}\right)^{\times}-J\left(A^{T}(\epsilon) \epsilon^{\times} e_{2}\right)^{\times}-\left(A^{T}(\epsilon) \epsilon^{\times} e_{2}\right)^{\times} J\right) e_{1} \\
& +6 \omega_{0}^{2} e_{1}^{T}\left(\left(J e_{3}\right)^{\times}-e_{3}^{\times} J\right)\left(A^{T}(\epsilon)-I\right) \epsilon^{\times} e_{3} \\
& +12 \omega_{0}^{2} e_{1}^{T}\left(A^{T}(\epsilon) \epsilon^{\times} e_{3}\right)^{\times} J A^{T}(\epsilon) \epsilon^{\times} e_{3} \\
& -2 \omega_{0}^{2} e_{1}^{T}\left(\left(J e_{2}\right)^{\times}-e_{2}^{\times} J\right)\left(A^{T}(\epsilon)-I\right) \epsilon^{\times} e_{2} \\
& -4 \omega_{0}^{2} e_{1}^{T}\left(A^{T}(\epsilon) \epsilon^{\times} e_{2}\right)^{\times} J A^{T}(\epsilon) \epsilon^{\times} e_{2} \\
& h\left(x_{1}, x_{2}\right)=\left[\begin{array}{c}
(A(\epsilon)-I)\left[e_{2} e_{3}\right] x_{2} / 2 \\
b\left(x_{1}, x_{2}\right)
\end{array}\right] \\
& b\left(x_{1}, x_{2}\right)=\frac{J_{y}-J_{z}}{J_{x}} \omega_{y} \omega_{z}+2 \omega_{0} e_{1}^{T} J^{-1}\left(\left(J A^{T}(\epsilon) \epsilon^{\times} e_{2}\right)^{\times}\right. \\
& \left.-J\left(A^{T}(\epsilon) \epsilon^{\times} e_{2}\right)^{\times}-\left(A^{T}(\epsilon) \epsilon^{\times} e_{2}\right)^{\times} J\right)\left[e_{2} e_{3}\right] x_{2}, \\
& d=\left[\begin{array}{llll}
0 & 0 & 0 & \tau_{d x} / J_{x}
\end{array}\right]^{T}
\end{aligned}
$$

Using Proposition 7, it is clear that $g\left(x_{1}\right)$ is second order in $x_{1}$.

Now, suppose that $x_{2}$ can be used as a control input for the unactuated states $x_{1}$. Using the Popov-Belevitch-Hautus test [Zhou and Doyle, 1998], it can be seen that 
the pair $\left(\bar{A}_{11}, \bar{A}_{12}\right)$ is controllable if and only if the matrix $\left[\bar{A}_{11}-\lambda I \bar{A}_{12}\right]$ has full rank for all $\lambda \in \mathbb{C}$. Consequently, let $K \in \mathbb{R}^{2 \times 4}$ be such that $\bar{A}_{11}-\bar{A}_{12} K$ is Hurwitz. Next, we define the sliding variable

$$
S=x_{2}+K x_{1}
$$

Rearranging for $x_{2}$, we have $x_{2}=S-K x_{1}$, and substituting into (3.21), we obtain

$$
\dot{x}_{1}=\left(\bar{A}_{11}-\bar{A}_{12} K\right) x_{1}+\bar{A}_{12} S+g\left(x_{1}\right)+h\left(x_{1}, S-K x_{1}\right)+d .
$$

Furthermore, from the definition of $h\left(x_{1}, x_{2}\right)$ in (3.21)it can be seen that

$$
h\left(x_{1}, S-K x_{1}\right)=h_{1}\left(x_{1}\right)+h_{2}\left(x_{1}, S\right)
$$

where $h_{1}\left(x_{1}\right)$ is second order in $x_{1}$, and $h_{2}\left(x_{1}, S\right)$ satisfies

$$
\left\|h_{2}\left(x_{1}, S\right)\right\| \leq\left(a\left\|x_{1}\right\|+b\left\|x_{1}\right\|^{2}\right)\|S\|+c\|S\|^{2},
$$

for some $a, b, c>0$.

Since $\bar{A}_{11}-\bar{A}_{12} \mathrm{~K}$ is Hurwitz, for any $Q=Q^{T}>0$, there exists a $P=P^{T}>0$ such that $\left(\bar{A}_{11}-\bar{A}_{12} K\right)^{T} P+P\left(\bar{A}_{11}-\bar{A}_{12} K\right)=-Q$ [Zhou and Doyle, 1998]. Fixing $Q=Q^{T}>0$, consider the positive-definite function

$$
V_{1}\left(x_{1}\right)=x_{1}^{T} P x_{1}
$$

Taking the time-derivative of $V_{1}$ along trajectories of (3.24), leads to

$$
\begin{aligned}
\dot{V}_{1}= & -x_{1}^{T} Q x_{1}+2 x_{1}^{T} P\left(\bar{A}_{12} S+d\right)+2 x_{1}^{T} P\left(g\left(x_{1}\right)+h_{1}\left(x_{1}\right)\right)+2 x_{1}^{T} P h_{2}\left(x_{1}, S\right), \\
\leq & -\lambda_{\min }(Q)\left\|x_{1}\right\|^{2}+2\|P\|\left\|x_{1}\right\|\left(\left\|\bar{A}_{12}\right\|\|S\|+\|d\|\right)+2\|P\|\left\|x_{1}\right\|\left\|g\left(x_{1}\right)+h_{1}\left(x_{1}\right)\right\| \\
& +2\|P\|\left(\left(a\left\|x_{1}\right\|^{2}+b\left\|x_{1}\right\|^{3}\right)\|S\|+c\left\|x_{1}\right\|\|S\|^{2}\right) .
\end{aligned}
$$

Suppose that $\|S\| \leq \bar{S}$ and $\|d\| \leq \bar{d}$, for some $0<\bar{S} \leq 1, \bar{d}>0$. Then, $\|S\|^{2} \leq\|S\| \leq$ $\bar{S}$. Likewise, if the domain of $x_{1}$ is restricted to $\left\|x_{1}\right\|<1$, one has $\left\|x_{1}\right\|^{3} \leq\left\|x_{1}\right\|^{2} \leq$ 
$\left\|x_{1}\right\|$. Consequently,

$$
\dot{V}_{1} \leq-\lambda_{\min }(Q)\left\|x_{1}\right\|^{2}+2\|P\|\left\|x_{1}\right\|(\Gamma \bar{S}+\bar{d})+2\|P\|\left\|x_{1}\right\|\left\|g\left(x_{1}\right)+h_{1}\left(x_{1}\right)\right\|
$$

where $\Gamma=\left\|\bar{A}_{12}\right\|+a+b+c$. Next, since $g\left(x_{1}\right)$ and $h\left(x_{1}\right)$ are second order in $x_{1}$, given any $v>0$, there exists $\delta>0$ such that $\left\|g\left(x_{1}\right)+h_{1}\left(x_{1}\right)\right\| \leq v\left\|x_{1}\right\|$, for any $x_{1}$ satisfying $\left\|x_{1}\right\|<\delta$. Consequently, for all $x_{1}$ satisfying $\left\|x_{1}\right\|<\min \{\delta, 1\}$,

$$
\dot{V}_{1} \leq-\left(\lambda_{\min }(Q)-2\|P\| v\right)\left\|x_{1}\right\|^{2}+2\|P\|\left\|x_{1}\right\|(\Gamma \bar{S}+\bar{d})
$$

We choose $0<v<\lambda_{\min }(Q) /(2\|P\|)$. Next, using the upper bound of

$$
\lambda_{\min }(P)\left\|x_{1}\right\|^{2} \leq V_{1}\left(x_{1}\right) \leq \lambda_{\max }(P)\left\|x_{1}\right\|^{2},
$$

we have

$$
\begin{aligned}
\dot{V}_{1} & \leq-\frac{\left(\lambda_{\min }(Q)-2\|P\| v\right)}{\lambda_{\max }(P)} V_{1}+\sqrt{V_{1}} \frac{2\|P\|(\Gamma \bar{S}+\bar{d})}{\sqrt{\lambda_{\min }(P)}} \\
& =-\sqrt{V_{1}}\left(\frac{\left(\lambda_{\min }(Q)-2\|P\| v\right)}{\|P\|} \sqrt{V_{1}}-\frac{2\|P\|(\Gamma \bar{S}+\bar{d})}{\sqrt{\lambda_{\min }(P)}}\right),
\end{aligned}
$$

for all $x_{1}$ satisfying $\left\|x_{1}\right\|<\min \{\delta, 1\}$. From (3.28), one sees that $\dot{V}_{1}<0$ when

$$
V_{1}\left(x_{1}\right)>\left(\frac{2\|P\|^{2}(\Gamma \bar{S}+\bar{d})}{\sqrt{\lambda_{\min }(P)}\left(\lambda_{\min }(Q)-2\|P\| v\right)}\right)^{2}
$$

However, recall that this only holds when $\left\|x_{1}\right\|<\min \{\delta, 1\}$. By (3.27),

$$
V_{1}\left(x_{1}\right)<\lambda_{\min }(P)(\min \{\delta, 1\})^{2},
$$

implies that $\left\|x_{1}\right\|<\min \{\delta, 1\}$. Consequently, to guarantee feasibility for (3.29), the following restriction is made:

$$
\frac{2\|P\|^{2}(\Gamma \bar{S}+\bar{d})}{\left(\lambda_{\min }(Q)-2\|P\| v\right)}<\lambda_{\min }(P) \min \{\delta, 1\} .
$$


This is clearly satisfied if $\bar{S}$ and $\bar{d}$ are small enough. The following preliminary result is obtained.

Lemma 3.1. Suppose that (3.30) holds, and that $\|S(t)\| \leq \bar{S}, d(t) \leq \bar{d}$ for all $t \in[0, T)$, for some $T \in \mathbb{R}_{+} \bigcup\{\infty\}$. If $x_{1}(0)$ satisfies $V_{1}\left(x_{1}(0)\right)<\lambda_{\min }(P)(\min \{\delta, 1\})^{2}$, then $V\left(x_{1}(t)\right)<\lambda_{\min }(P)(\min \{\delta, 1\})^{2}$ for all $t \in[0, T)$. If $T=\infty$, and $\lim \sup _{t \rightarrow \infty}\|S(t)\| \leq$ $S_{\ell}<\bar{S}$, then

$$
\limsup _{t \rightarrow \infty}\left\|x_{1}(t)\right\| \leq \frac{2\|P\|^{2}\left(\Gamma S_{\ell}+\bar{d}\right)}{\lambda_{\min }(P)\left(\lambda_{\min }(Q)-2\|P\| v\right)} .
$$

Proof. First, note that $\dot{V}_{1}<0$ when (3.29) holds. Consequently,

$V_{1}\left(x_{1}(t)\right) \leq \min \left\{\left(\frac{2\|P\|^{2}(\Gamma \bar{S}+\bar{d})}{\sqrt{\lambda_{\min }(P)}\left(\lambda_{\min }(Q)-2\|P\| v\right)}\right)^{2}, V_{1}\left(x_{1}(0)\right)\right\}<\lambda_{\min }(P)(\min \{\delta, 1\})^{2}$,

for all $t \in[0, T)$. Next, for any $\alpha>0$ satisfying $S_{\ell}+\alpha<\bar{S}$, by definition of $S_{\ell}$, there exists $\bar{t} \geq 0$, such that for all $t \geq \bar{t},\|S(t)\| \leq S_{\ell}+\alpha / 2$. Consequently, in (3.28) one can replace $\bar{S}$ by $S_{\ell}+\alpha / 2$ from time $\bar{t}$ onwards. If

$$
V_{1}\left(x_{1}(\bar{t})\right) \leq\left(\frac{2\|P\|^{2}\left(\Gamma\left(S_{\ell}+\alpha\right)+\bar{d}\right)}{\sqrt{\lambda_{\min }(P)}\left(\lambda_{\min }(Q)-2\|P\| v\right)}\right)^{2}
$$

then,

$$
V_{1}\left(x_{1}(t)\right) \leq\left(\frac{2\|P\|^{2}\left(\Gamma\left(S_{\ell}+\alpha\right)+\bar{d}\right)}{\sqrt{\lambda_{\min }(P)}\left(\lambda_{\min }(Q)-2\|P\| v\right)}\right)^{2},
$$

for all $t \geq \bar{t}$, since $\dot{V}_{1}<0$ on the boundary of this set. Define the set

$$
E \triangleq\left\{x_{1} \in \mathbb{R}^{4}:\left(\frac{2\|P\|^{2}\left(\Gamma\left(S_{\ell}+\alpha\right)+\bar{d}\right)}{\sqrt{\lambda_{\min }(P)}\left(\lambda_{\min }(Q)-2\|P\| v\right)}\right)^{2}<V_{1}\left(x_{1}\right)<\lambda_{\min }(P)(\min \{\delta, 1\})^{2}\right\} .
$$

Now consider the case that $x_{1}(\bar{t})$ lies in the interior of $E$. In this case, since $E$ is compact, and the right-hand side of (3.28) is strictly negative on $E$, there exists a $\beta>0$ such that $\dot{V}_{1} \leq-\beta$ for all $x_{1} \in E$. Consequently, there exists a finite time $t^{\prime}>\bar{t}$, such that

$$
V_{1}\left(x_{1}(t)\right) \leq\left(\frac{2\|P\|^{2}\left(\Gamma\left(S_{\ell}+\alpha\right)+\bar{d}\right)}{\sqrt{\lambda_{\min }(P)}\left(\lambda_{\min }(Q)-2\|P\| v\right)}\right)^{2}
$$


for all $t \geq t^{\prime}$. Since $\alpha>0$ was arbitrary, it follows that

$$
\limsup _{t \rightarrow \infty} V_{1}\left(x_{1}(t)\right) \leq\left(\frac{2\|P\|^{2}\left(\Gamma S_{\ell}+\bar{d}\right)}{\sqrt{\lambda_{\min }(P)}\left(\lambda_{\min }(Q)-2\|P\| v\right)}\right)^{2} .
$$

Applying (3.27) yields the desired expression for lim $\sup _{t \rightarrow \infty}\left\|x_{1}(t)\right\|$. This concludes the proof.

\subsubsection{Control design for actuated states}

Now that the result in Lemma 3.1 has been established, the control design task is to guarantee that $\|S(t)\| \leq \bar{S}$ for all $t \geq 0$. Note that the sliding variable $S$ in (3.23) can be rewritten as

$$
S=K_{1} \omega_{b n}+K_{2} \epsilon,
$$

where $K_{1}=\left[\begin{array}{ll}k_{4} & I\end{array}\right], K_{2}=\left[\begin{array}{lll}k_{1} & k_{2} & k_{3}\end{array}\right]$, and $k_{i}$ denotes the $i^{t h}$ column of $K$. Defining the reduced control torque

$$
\bar{\tau}_{u}=\left[\tau_{y}, \tau_{z}\right]^{T},
$$

differentiating (3.31) and utilizing (3.9) and (3.15), leads to

$$
\dot{S}=\Omega \bar{\tau}_{u}+\xi(\epsilon, \eta)
$$

where

$$
\Omega=K_{1} J^{-1}\left[\begin{array}{ll}
0 & 0 \\
1 & 0 \\
0 & 1
\end{array}\right]
$$

and

$$
\begin{aligned}
\xi(\epsilon, \eta)= & K_{1} J^{-1}\left(-\omega_{b n}^{\times} J \omega_{b n}+\omega_{0}\left(\omega_{b n}^{\times} J-J \omega_{b n}^{\times}-\left(J \omega_{b n}\right)^{\times}\right) C_{b n} e_{2}+3 \omega_{0}^{2}\left(C_{b n} e_{3}\right)^{\times} J C_{b n} e_{3}\right. \\
& \left.-\omega_{0}^{2}\left(C_{b n} e_{2}\right)^{\times} J C_{b n} e_{2}+\tau_{d}\right)+K_{2} A(\epsilon) \omega_{b n} / 2 .
\end{aligned}
$$

We find that $\left\|\xi\left(\epsilon, \omega_{b n}\right)\right\| \leq \chi_{1}+\chi_{2}\left\|\omega_{b n}\right\|+\chi_{3}\left\|\omega_{b n}\right\|^{2} \triangleq \phi$, for some $\chi_{1}, \chi_{2}, \chi_{3}>0$. 


\section{Full-state feedback control}

To motivate the velocity-free case, the full state feedback case is considered first.

Define the control law to be

$$
\bar{\tau}_{u}=\Omega^{-1} \bar{u}
$$

where

$$
\bar{u}=-(\sigma S+\psi)
$$

with $\sigma>0$ and

$$
\psi=\phi \tanh \left(\frac{2 \phi k_{u} S}{\bar{\delta}}\right)
$$

where $\bar{\delta}>0$ and $k_{u}=0.2785$. One has the following property [Polycarpou and Ioannou, 1996]:

$$
S^{T} \xi-S^{T} \psi \leq \bar{\delta}
$$

Lemma 3.2. With the control law in (3.35), one has $\|S(t)\| \leq \min \{\|S(0)\|, \sqrt{\bar{\delta} / \sigma}\}$, and

$$
\limsup _{t \rightarrow \infty}\|S(t)\| \leq \sqrt{\bar{\delta} / \sigma}
$$

Proof. Define $V_{S}(S)=\frac{1}{2} S^{T} S$. Differentiating along a trajectory of (3.33) with control law (3.35), and using (3.36) one obtains $\dot{V}_{s} \leq-2 \sigma V_{s}+\bar{\delta}$. The conclusion can be reached by applying similar arguments as in the proof of Lemma 3.1.

The main result for the full state feedback case can now be presented.

Theorem 3.3. Suppose that (3.30) holds. Select $\bar{\delta}, \sigma$ in (3.35), such that $\bar{\delta} / \sigma<\bar{S}$. Then, provided $x_{1}(0)$ and $x_{2}(0)$ are chosen such that $V_{1}\left(x_{1}(0)\right)<\lambda_{\min }(P)(\min \{\delta, 1\})^{2}$ and $\|S(0)\| \leq \bar{S}$, the following holds

$$
\limsup _{t \rightarrow \infty}\left\|x_{1}(t)\right\| \leq \frac{2\|P\|^{2}(\Gamma \sqrt{\bar{\delta} / \sigma}+\bar{d})}{\lambda_{\min }(P)\left(\lambda_{\min }(Q)-2\|P\| v\right)}
$$

Proof. This follows immediately from Lemmas 3.1 and 3.2. 


\section{Velocity-free feedback control}

This subsection investigates the case where only the attitude error quaternion $(\epsilon, \eta) \in \mathbb{S}^{3}$ is available. The relative angular velocity $\omega_{b n}$ is replaced in the control law (3.35) by an estimate $\hat{\omega}_{b n}$ which comes from a finite-time observer that is defined in the next section. The observer will actually provide an estimate $\hat{\omega}_{b I}$ of the spacecraft inertial angular velocity $\hat{\omega}_{b I}$, such that the estimate $\hat{\omega}$ is obtained from

$$
\hat{\omega}_{b n}=\hat{\omega}_{b I}+\omega_{0} C_{b n} e_{2}
$$

The estimation error of $\hat{\omega}_{b I}$ is defined as

$$
\tilde{\omega}=\omega_{b I}-\hat{\omega}_{b I}
$$

Consequently, since $\omega_{b n}=\omega_{b I}+\omega_{0} C_{b n} e_{2}$, one has

$$
\hat{\omega}_{b n}=\omega_{b n}-\tilde{\omega}
$$

Replacing $\omega_{b n}$ with $\hat{\omega}_{b n}$ in (3.35) results in the control law

$$
\bar{u}=-(\sigma \hat{S}+\hat{\psi})
$$

where

$$
\hat{S}=K_{1} \hat{\omega}_{b n}+K_{2} \epsilon, \quad \hat{\psi}=\hat{\phi} \tanh \left(\frac{2 \hat{\phi} k_{u} \hat{S}}{\bar{\delta}}\right), \hat{\phi}=\chi_{1}+\chi_{2}\left\|\hat{\omega}_{b n}\right\|+\chi_{3}\left\|\hat{\omega}_{b n}\right\|^{2}
$$

Recall that $x=\left[\begin{array}{ll}\epsilon^{T} & \omega_{b n}^{T}\end{array}\right]^{T}$. Using (3.35), (3.39) and (3.40), the control input (3.40) can be rewritten as

$$
\bar{u}=-(\sigma S+\psi)+\Delta \bar{u}(x, \tilde{w})
$$

where

$$
\Delta \bar{u}(x, \tilde{\omega})=\sigma K_{1} \tilde{\omega}+\psi-\hat{\psi},
$$

noting that $\psi$ in (3.35) is a function of $x$, while $\hat{\psi}$ in (3.40) can be viewed as a function of $(x, \tilde{\omega})$ because of (3.39). Next, note that $\Delta \bar{u}$ in (3.43) is continuous in $(x, \tilde{\omega})$ on 
$\mathbb{R}^{6} \times \mathbb{R}^{3}$. Furthermore, $\Delta \bar{u}(x, 0)=0$ for all $x \in \mathbb{R}^{6}$.

Proposition 8. Fix $r>0$ and define the set $W=\left\{(x, \tilde{\omega}) \in \mathbb{R}^{6} \times \mathbb{R}^{3}:\|x\| \leq r, \tilde{\omega}=\right.$ $0\}$. Then, for any $\rho>0$ there exists an $\varrho(r)>0$ such that $\|\tilde{\omega}\|<\varrho(r)$ implies that $\|\Delta \bar{u}(x, \tilde{\omega})\|<\rho$ for all $x$ satisfying $\|x\| \leq r$.

Proof. First, note that the set $W$ is compact. Next, choose arbitrary $\rho>0$. Continuity of $\Delta \bar{u}$ implies that for each point $(x, 0) \in W$, there exists $\varrho_{x}>0$ such that $\|\Delta \bar{u}(y, \tilde{\omega})\|<\rho$ for all $(y, \tilde{\omega}) \in B_{Q_{x}}(x) \times B_{Q_{x}}(0)$ (where $B_{\epsilon}(x)$ denotes the open ball of radius $\epsilon$ centered on $x$ ). Next, the collection of all such sets $B_{Q_{x}}(x) \times B_{Q_{x}}(0)$ (with $x \in$ $\left.\bar{B}_{r}(0)\right)$ form an open cover of $W$. Hence, since $W$ is compact, there exist $x_{1}, \ldots, x_{n} \in$ $\bar{B}_{r}(0)$, such that $W \subset \bigcup_{i=1}^{n} B_{Q_{x i}}\left(x_{i}\right) \times B_{Q_{x i}}(0)$. By construction, $\|\Delta \bar{u}(y, \tilde{\omega})\|<\rho$ for all $(y, \tilde{\omega}) \in \bigcup_{i=1}^{n} B_{\varrho_{x i}}\left(x_{i}\right) \times B_{\varrho_{x i}}(0)$. Define $\varrho(r)=\min \left\{\varrho_{x 1}, \ldots, \varrho_{x n}\right\}$. Then, $\bar{B}_{r}(0) \times B_{\varrho(r)}(0) \subset \bigcup_{i=1}^{n} B_{\varrho_{x i}}\left(x_{i}\right) \times B_{\varrho_{x i}}(0)$, from which the conclusion follows.

Using Proposition 8, one obtains the following extension of Lemma 3.2.

Lemma 3.4. Fix $r>0$, and suppose that $\|x(t)\| \leq r$ for all $t \in[0, \bar{t})$, where $\bar{t} \in \mathbb{R}_{+} \bigcup\{\infty\}$. Then, for a given $\rho>0$, there exists $\varrho(r)>0$ such that $\|\tilde{\omega}(t)\|<\varrho(r)$ for all $t \geq[0, \bar{t})$ implies that $\|S(t)\| \leq \min \left\{\|S(0)\|,\left(\rho+\sqrt{\rho^{2}+4 \sigma \bar{\delta}}\right) /(2 \sigma)\right\}$ for all $t \geq[0, \bar{t})$. Furthermore, if $\bar{t}=\infty$, then

$$
\limsup _{t \rightarrow \infty}\|S(t)\| \leq \frac{\rho+\sqrt{\rho^{2}+4 \sigma \bar{\delta}}}{2 \sigma}
$$

Proof. Define $V_{S}(S)=\frac{1}{2} S^{T} S$. Differentiating along a trajectory of (3.33) with control law (3.42), one obtains $\dot{V}_{s} \leq-2 \sigma V_{s}+\bar{\delta}+S^{T} \Delta \bar{u}$. Using Proposition 8, one obtains for $t \in[0, \bar{t})$ that $\dot{V}_{s} \leq-2 \sigma V_{s}+\rho \sqrt{2} \sqrt{V_{s}}+\bar{\delta}$. From this, it can be obtained that $\dot{V}_{s}<0$ when

$$
V_{s}>\frac{1}{8}\left(\frac{\rho+\sqrt{\rho^{2}+4 \sigma \bar{\delta}}}{\sigma}\right)^{2}
$$

The conclusion follows by applying similar arguments as in the proof of Lemma 3.1.

\subsubsection{Observer design}

Let $q=\left(q_{v}, q_{4}\right) \in \mathbb{S}^{3}$ be a unit quaternion representation of the spacecraft inertial attitude (note that this is directly related to $(\epsilon, \eta)$, since the orbiting frame is known). 
The spacecraft equations of motion relative to the inertial frame are then given by

$$
\begin{aligned}
\dot{q} & =\frac{1}{2} B(q) \omega_{b I}, \\
J \dot{\omega}_{b I} & =-\omega_{b I}^{\times} J \omega_{b I}+\tau_{g}+\tau_{u}+\tau_{d},
\end{aligned}
$$

where

$$
B(q)=\left[\begin{array}{c}
P(q) \\
-q_{v}^{T}
\end{array}\right], P(q)=q_{4} I+q_{v}^{\times} .
$$

Let $\hat{q}, \hat{\omega}_{b I}$ be the estimates of $q, \omega_{b I}$ respectively, and define the attitude estimation error $\tilde{q}=\left(\tilde{q}_{v}, \tilde{q}_{4}\right)$ by $C(\tilde{q})=C(q) C^{T}(\hat{q})$ and $\tilde{\omega}=\omega_{b I}-\hat{\omega}_{b I}$, where

$$
C(q)=\left(q_{4}^{2}-q_{v}^{T} q_{v}\right) I+2 q_{v} q_{v}^{T}-q_{4} q_{v}^{\times}
$$

is the rotation matrix corresponding to $q$. Let $\hat{q}$ satisfy

$$
\dot{\hat{q}}=\frac{1}{2} B(\hat{q}) p
$$

for some $p$, or equivalently [Hughes, 1986], $\dot{C}(\hat{q})=-p^{\times} C(\hat{q})$. Consequently, it is straightforward to show that $\dot{C}(\tilde{q})=-\left(\omega_{b I}-C(\tilde{q}) p\right)^{\times} C(\tilde{q})$, which is equivalent to

$$
\dot{\tilde{q}}=B(\tilde{q})\left(\omega_{b I}-C(\tilde{q}) p\right)
$$

The observer is now defined as

$$
\begin{aligned}
\dot{\hat{q}} & =\frac{1}{2} B(\hat{q}) C^{T}(\tilde{q})\left(\hat{\omega}_{b I}+\gamma_{1} P^{-1}(\tilde{q}) \operatorname{sig}^{\alpha}\left(\tilde{q}_{v}\right)\right), \\
J \dot{\hat{\omega}}_{b I} & =-\hat{\omega}_{b I}^{\times} J \hat{\omega}_{b I}+\tau_{g}+\tau_{u}+\gamma_{2} J \operatorname{sig}^{\alpha_{1}}\left(\tilde{q}_{v}\right),
\end{aligned}
$$

where $\gamma_{1}, \gamma_{2}>0, \alpha \in(1 / 2,1)$, and $\alpha_{1}=2 \alpha-1$. Note that $P^{-1}(\tilde{q})$ is invertible provided $\left\|\tilde{q}_{v}\right\|<1$. Since a local stability result is obtained, this restriction poses no problem. Utilizing (3.45), (3.47), (3.48), (3.49), and (3.50), one obtains the error 
equations

$$
\begin{aligned}
\dot{\tilde{q}}_{v} & =\frac{1}{2} B(\tilde{q})\left(\tilde{\omega}-\gamma_{1} P^{-1}(\tilde{q}) \operatorname{sig}^{\alpha}\left(\tilde{q}_{v}\right)\right), \\
\dot{\tilde{\omega}} & =-\gamma_{2} \operatorname{sig}^{\alpha_{1}}\left(\tilde{q}_{v}\right)-J^{-1}\left(\omega_{b I}^{\times} J \omega_{b I}-\hat{\omega}_{b I}^{\times} J \hat{\omega}_{b I}\right)+J^{-1} \tau_{d} .
\end{aligned}
$$

Next, using the definition of $\tilde{\omega}$, it is straightforward to show that

$$
\omega_{b I}^{\times} J \omega_{b I}-\hat{\omega}_{b I}^{\times} J \hat{\omega}_{b I}=\tilde{\omega}^{\times} J \omega_{b I}+\omega_{b I}^{\times} J \tilde{\omega}-\tilde{\omega}^{\times} J \tilde{\omega} .
$$

Finally, since a local stability result is pursued, with the assumption that $\left\|\tilde{q}_{v}\right\|<1$, choose $\tilde{q}_{4}>0$. Defining $\tilde{x}=\left[\tilde{q}_{v}^{T}, \tilde{\omega}^{T}\right]^{T}$, the observer error dynamics in (3.51) and (3.52) can be written as

$$
\dot{\tilde{x}}=g_{\alpha}(\tilde{x})+f\left(\tilde{q}_{v}, \tilde{\omega}, \omega_{b I}\right)+\tilde{d},
$$

where

$$
\begin{gathered}
g_{\alpha}(\tilde{x})=\left[\begin{array}{l}
g_{1 \alpha}(\tilde{x}) \\
g_{2 \alpha}(\tilde{x})
\end{array}\right], f\left(\tilde{q}_{v}, \tilde{\omega}, \omega_{b I}\right)=\left[\begin{array}{c}
f_{1}\left(\tilde{q}_{v}, \tilde{\omega}\right) \\
f_{2}\left(\tilde{\omega}, \omega_{b I}\right)
\end{array}\right], \tilde{d}=\left[\begin{array}{c}
0 \\
J^{-1} \tau_{d}
\end{array}\right], \\
g_{1 \alpha}(\tilde{x})=\tilde{\omega} / 2-\gamma_{1} \operatorname{sig}^{\alpha}\left(\tilde{q}_{v}\right), g_{2 \alpha}(\tilde{x})=-\gamma_{2} \operatorname{sig}^{\alpha_{1}}\left(\tilde{q}_{v}\right) \\
f_{1}\left(\tilde{q}_{v}, \tilde{\omega}\right)=\frac{1}{2}\left(A\left(\tilde{q}_{v}\right)-I\right) \tilde{\omega}, \\
f_{2}\left(\tilde{\omega}, \omega_{b I}\right)=-J^{-1}\left(\tilde{\omega}^{\times} J \omega_{b I}+\omega_{b I}^{\times} J \tilde{\omega}-\tilde{\omega}^{\times} J \tilde{\omega}\right) .
\end{gathered}
$$

It can be verified that $g_{\alpha}$ is homogeneous of degree $k=\alpha-1$ with respect to the dilation $\delta_{\lambda}^{r}(\tilde{x})=\left[\lambda \tilde{q}_{v}^{T}, \lambda^{\alpha} \tilde{\omega}^{T}\right]^{T}$.

A strict Lyapunov function will now be constructed for the nominal system corresponding to (3.53) (with $f \equiv \tilde{d} \equiv 0$ ). For the purposes of Lyapunov function construction, the requirement on $\alpha$ will be relaxed to $\alpha \in(1 / 2, \infty)$. When $\alpha=1$, the vector field $g_{\alpha}(\tilde{x})$ becomes

$$
g_{\alpha=1}(\tilde{x})=M \tilde{x},
$$


where

$$
M=\left[\begin{array}{cc}
-\gamma_{1} I & I / 2 \\
-\gamma_{2} I & 0
\end{array}\right] \text {. }
$$

This is Hurwitz for any $\gamma_{1}, \gamma_{2}>0$. Consequently, for fixed $\gamma_{1}, \gamma_{2}>0$ there exists a positive-definite symmetric matrix $N \in \mathbb{R}^{6 \times 6}$ such that

$$
M^{T} N+N M=-I
$$

Next, define $\bar{V}(\tilde{x})=\tilde{x}^{T} N \tilde{x}$. Note that this is infinitely continuously differentiable, positive-definite and radially unbounded. Furthermore, by (3.55), one has

$$
L_{g_{\alpha=1}} \bar{V}(\tilde{x})=-\|\tilde{x}\|^{2}<0
$$

The procedure in Rosier [Rosier, 1992] is now used to construct a homogeneous Lyapunov function from $\bar{V}$. To this end, define

$$
V_{\alpha}(\tilde{x})=\int_{0^{+}}^{\infty} \frac{1}{t^{\ell+1}} a\left(\bar{V}\left(\delta_{t}^{r(\alpha)}(\tilde{x})\right)\right) d t
$$

where $\ell \geq 2$ and $a \in \mathcal{C}^{\infty}(\mathbb{R}, \mathbb{R})$ satisfies

$$
a(t)= \begin{cases}0, & t \in(-\infty, 1] \\ 1, & t \in[2, \infty)\end{cases}
$$

with $d a / d t \geq 0$ for all $t \in \mathbb{R}$. From the results in Rosier [Rosier, 1992], one obtains the following properties of $V_{\alpha}$ :

Proposition 9. $V_{\alpha}: \mathbb{R}^{n} \rightarrow \mathbb{R}$ in (3.57) is well-defined and satisfies the following properties:

1. $V_{\alpha}$ is positive-definite and radially unbounded.

2. $V_{\alpha}$ is homogeneous of degree $\ell$ with respect to the dilation $\delta_{\lambda}^{r(\alpha)}$.

3. $V_{\alpha}$ is infinitely differentiable on $\mathbb{R}^{n} \backslash\{0\}$ and continuously differentiable at $\tilde{x}=0$.

Next, the Lie derivative of $V_{\alpha}$ along $g_{\alpha}$ is examined. Since $g_{\alpha}(0)=0$, it is clear that $L_{g_{\alpha}} V_{\alpha}(0)=0$. Hence, focus on $L_{g_{\alpha}} V_{\alpha}(\tilde{x})$ for $x \neq 0$. By [Rosier (1992)], any $\tilde{x} \neq 0$ can be written as $\delta_{\lambda}^{r(\alpha)}(\bar{x})$, for some $\lambda>0$ and $\bar{x} \in \mathbb{S}^{5}$. By Proposition $4, L_{g_{\alpha}} V_{\alpha}$ is 
homogeneous of degree $\ell+k$ with respect to the dilation $\delta_{\lambda}^{r(\alpha)}$. Hence, for any $\tilde{x} \neq 0$,

$$
L_{g_{\alpha}} V_{\alpha}(\tilde{x})=L_{g_{\alpha}} V_{\alpha}\left(\delta_{\lambda}^{r(\alpha)}(\bar{x})\right)=\lambda^{\ell+k} L_{g_{\alpha}} V_{\alpha}(\bar{x})
$$

for some $\lambda>0$ and $\bar{x} \in \mathbb{S}^{5}$, indicating that to show $L_{g_{\alpha}} V_{\alpha}(\tilde{x})<0$ for all $\tilde{x} \neq 0$, it suffices to show that $L_{g_{\alpha}} V_{\alpha}(\tilde{x})<0$ for all $\tilde{x} \in \mathbb{S}^{5}$.

Proposition 10. Choose $0<a<1<b$. Then, there exist $0<l<L$ the following hold:

1. $\bar{V}\left(\delta_{t}^{r(\alpha)}(\alpha)(x)\right)<1$ for all $0<t<l$

2. $\bar{V}\left(\delta_{t}^{r(\alpha)}(\alpha)(x)\right)>2$ for all $t>L$

3.

$$
V_{\alpha}(x)=\int_{l}^{L} \frac{1}{t^{\ell+1}} a\left(\bar{V}\left(\delta_{t}^{r(\alpha)}(x)\right)\right) d t+\frac{2}{k L^{k}} .
$$

Proof. Consider the set $A=\left\{\tilde{x}=(y, z) \in \mathbb{R}^{3} \times \mathbb{R}^{3}: a \leq\|\tilde{x}\| \leq b\right\}$. Note that by equivalence of norms on $\mathbb{R}^{6}$, there exist $\zeta, \beta>0$ such that $\zeta\|\tilde{x}\|_{1} \leq\|\tilde{x}\| \leq \beta\|\tilde{x}\|_{1}$. Hence, for $t>0$,

$$
\zeta\left(t\|y\|_{1}+t^{\alpha}\|z\|_{1}\right) \leq\left\|\delta_{t}^{r(\alpha)}(\tilde{x})\right\| \leq \beta\left(t\|y\|_{1}+t^{\alpha}\|z\|_{1}\right)
$$

which leads to

$$
\frac{\zeta}{\beta} \min \left\{t, t^{\alpha}\right\}\|\tilde{x}\| \leq\left\|\delta_{t}^{r(\alpha)}(\tilde{x})\right\| \leq \frac{\beta}{\zeta} \max \left\{t, t^{\alpha}\right\}\|\tilde{x}\|
$$

Hence, for $\tilde{x} \in A$,

$$
\frac{\zeta}{\beta} a \min \left\{t, t^{\alpha}\right\} \leq\left\|\delta_{t}^{r(\alpha)}(\tilde{x})\right\| \leq \frac{\beta}{\zeta} b \max \left\{t, t^{\alpha}\right\}
$$

Next, note that

$$
\max \left\{t, t^{\alpha}\right\}<t^{1 / 2}, 0<t<1, \text { and } \min \left\{t, t^{\alpha}\right\}>t^{1 / 2}, t>1,
$$

for all $\alpha \in(1 / 2, \infty)$. By continuity of $\bar{V}(x)$ at $x=0$, there exists a $\delta>0$, such that $\|x\| \leq \delta$ implies that $V(x) \leq 1$. Likewise, by radial unboundedness of $\bar{V}(x)$, there 
exists an $r>0$ such that $\|x\| \geq r$ implies $\bar{V}(x) \geq 2$. By (3.60), it follows that there exists $0<\ell<L$, such that for all $\tilde{x} \in A$ and $\alpha \in(1 / 2, \infty), 0 \leq t \leq \ell$ implies that $\left\|\delta_{t}^{r(\alpha)}(\tilde{x})\right\| \leq \delta$, and $t \geq L$ implies that $\left\|\delta_{t}^{r(\alpha)}(\tilde{x})\right\| \geq r$. Hence, $\bar{V}\left(\delta_{t}^{r(\alpha)}(\tilde{x})\right) \leq 1$ when $0 \leq t \leq \ell$, and $\bar{V}\left(\delta_{t}^{r(\alpha)}(\tilde{x})\right) \geq 2$ when $t \geq L$, which are properties 1 and 2. Finally, property 3 is obtained by applying these two properties to (3.57). This concludes the proof.

Using Proposition 10, for $\tilde{x} \in \mathbb{S}^{5}$ and $\alpha \in(1 / 2, \infty)$,

$$
L_{g_{\alpha}} V_{\alpha}(\tilde{x})=\int_{l}^{L} \frac{1}{t^{\ell+1}} a^{\prime}\left(\bar{V}\left(\delta_{t}^{r(\alpha)}(\tilde{x})\right)\right) \frac{\partial \bar{V}}{\partial \tilde{x}}\left(\delta_{t}^{r(\alpha)}(\tilde{x})\right) \frac{\partial \delta_{t}^{r(\alpha)}}{\partial \tilde{x}}(\tilde{x}) g_{\alpha}(\tilde{x}) d t
$$

Next, the homogeneity of $g_{\alpha}$ of degree $\alpha-1$ with respect to dilation $\delta_{\lambda}^{r(\alpha)}$ implies that

$$
\frac{\partial \delta_{t}^{r(\alpha)}}{\partial x}(x) g_{\alpha}(x)=\frac{1}{t^{\alpha-1}} g_{\alpha}\left(\delta_{t}^{r(\alpha)}(\tilde{x})\right)
$$

Consequently,

$$
L_{g_{\alpha}} V_{\alpha}(\tilde{x})=\int_{l}^{L} \frac{1}{t^{\ell+\alpha}} a^{\prime}\left(\bar{V}\left(\delta_{t}^{r(\alpha)}(\tilde{x})\right)\right) L_{g_{\alpha}} \bar{V}\left(\delta_{t}^{r(\alpha)}(\tilde{x})\right) d t
$$

Now, define the function $h(\tilde{x}, t, \alpha)=L_{g_{\alpha}} \bar{V}\left(\delta_{t}^{r(\alpha)}(\tilde{x})\right)$, which is continuous on $\mathbb{R}^{6} \times$ $\mathbb{R}_{+} \times(1 / 2, \infty)$. By (3.56), $h(\tilde{x}, t, 1)<0$ for all $(\tilde{x}, t) \in \mathbb{S}^{5} \times[\ell, L]$. Consequently, since the set $S^{5} \times[\ell, L] \times\{1\}$ is compact, there exists $\varsigma<0$ such that $h(\tilde{x}, t, 1) \leq \varsigma$ for all $\tilde{x} \in \mathbb{S}^{5} \times[\ell, L]$. Choose any $\bar{\zeta}$ satisfying $\varsigma<\bar{\zeta}<0$. Then, in a similar manner to the proof of Proposition 8 , there exists $\bar{\alpha}$, satisfying $0<\bar{\alpha} \leq 1 / 2$, such that $h(\tilde{x}, t, \alpha)<$ $\bar{\zeta}<0$ for all $(\tilde{x}, t) \in \mathbb{S}^{5} \times[\ell, L]$ when $1-\bar{\alpha}<\alpha<1+\bar{\alpha}$. Finally, by properties 1 . and 2. of Proposition 10 and the definition of $a(t)$, for any $\tilde{x} \in \mathbb{S}^{5}$, the term $a^{\prime}\left(\bar{V}\left(\delta_{t}^{r(\alpha)}(\tilde{x})\right)\right)$ must be positive on some sub-interval of $[l, L]$ (otherwise $a(s)$ cannot transition from $0 \rightarrow 1$ as $s$ goes from $1 \rightarrow 2$ ). Consequently, (3.62) shows that $L_{g_{\alpha}} V_{\alpha}(\tilde{x})<0$ for all $\tilde{x} \in \mathbb{S}^{5}$, and as explained previously, this implies that $L_{g_{\alpha}} V_{\alpha}(\tilde{x})<0$ for all $\tilde{x} \neq 0$.

Applying Proposition 5, for $1 / 2 \leq \bar{\alpha}<\alpha<1$ there exists $c(\alpha)>0$ such that

$$
L_{g_{\alpha}} V_{\alpha}(\tilde{x}) \leq-c(\alpha) V_{\alpha}^{(\ell+k) / \ell}(\tilde{x})
$$

for all $\tilde{x} \in \mathbb{R}^{6}$. 
Having obtained a Lyapunov function for the nominal system given by $g_{\alpha}(\tilde{x})$, the remaining terms $f\left(\tilde{q}_{v}, \tilde{\omega}, \omega_{b I}\right)$ and $\tilde{d}$ in (3.53) are now dealt with. The term $f\left(\tilde{q}_{v}, \tilde{\omega}, \omega_{b I}\right)$ is analyzed first, following the approach in [Du and S. Li, 2012] and [Gui and Vukovich, 2016]. Suppose that there exists a $\lambda_{0}>0$, such that

$$
L_{f} V_{\alpha}\left(\delta_{\lambda}^{r(\alpha)}(\tilde{x})\right) \leq \lambda^{\ell+k} \frac{c(\alpha)}{2} d
$$

for all $\tilde{x} \in \mathbb{S}^{5}$, when $0<\lambda<\lambda_{0}$, where $d=\min _{\tilde{x} \in S^{5}} V_{\alpha}^{(\ell+k) / \ell}(\tilde{x})$. Define the set

$$
U=\left\{\tilde{x} \in \mathbb{R}: \tilde{x}=\delta_{\lambda}^{r(\alpha)}(\bar{x}), \bar{x} \in \mathbb{S}^{5}, 0 \leq \lambda<\lambda_{0}\right\}
$$

Proposition 11 (Gui et. al. (Gui, Vukovich, and S. Xu, 2016)). The set $U$ in (3.65) is open and contains the origin.

If (3.64) holds, then by the definition of $d$, we obtain

$$
L_{f} V_{\alpha}\left(\delta_{\lambda}^{r(\alpha)}(\tilde{x})\right) \leq \lambda^{\ell+k} \frac{c(\alpha)}{2} V_{\alpha}^{(\ell+k) / \ell}(\tilde{x})
$$

for all $\tilde{x} \in \mathbb{S}^{5}$, when $0<\lambda<\lambda_{0}$. Any $\tilde{x} \neq 0$ can be written as $\delta_{\lambda}^{r(\alpha)}(\bar{x})$, for some $\lambda>0$ and $\bar{x} \in \mathbb{S}^{5}$ [Rosier, 1992]. Hence, for arbitrary $\tilde{x} \neq 0$, (3.66) leads to

$$
\begin{aligned}
L_{f} V_{\alpha}(\tilde{x}) & =L_{f} V_{\alpha}\left(\delta_{\lambda}^{r(\alpha)}(\bar{x})\right) \leq \lambda^{\ell+k} \frac{c(\alpha)}{2} V_{\alpha}^{(\ell+k) / \ell}(\bar{x}) \\
& =\frac{c(\alpha)}{2}\left(\lambda^{\ell} V_{\alpha}(\bar{x})\right)^{(\ell+k) / \ell} \\
& =\frac{c(\alpha)}{2}\left(V_{\alpha}\left(\delta_{\lambda}^{r(\alpha)}(\bar{x})\right)\right)^{(\ell+k) / \ell}, \\
& =\frac{c(\alpha)}{2} V_{\alpha}^{(\ell+k) / \ell}(\tilde{x})
\end{aligned}
$$

where homogeneity of $V_{\alpha}$ has been applied in the fourth step. Continuity of $L_{f} V_{\alpha}$ and $V_{\alpha}$ imply that (3.67) holds for $\tilde{x}=0$ also. As a result, combining (3.63) and (3.67),

$$
L_{g_{\alpha}+f} V_{\alpha}(\tilde{x}) \leq-\frac{c(\alpha)}{2} V_{\alpha}^{(\ell+k) / \ell}(\tilde{x}), \forall \tilde{x} \in U
$$


It remains to establish the condition in (3.64). Note that (3.64) is equivalent to the existence of $\lambda_{0}>0$, such that

$$
\frac{L_{f} V_{\alpha}\left(\delta_{\lambda}^{r(\alpha)}(\tilde{x})\right)}{\lambda^{\ell+k}} \leq \frac{c(\alpha)}{2} d
$$

for all $\tilde{x} \in \mathbb{S}^{5}$, when $0<\lambda<\lambda_{0}$. For $\tilde{x}=\left[\tilde{\epsilon}^{T}, \tilde{\omega}^{T}\right]^{T} \in \mathbb{S}^{5}$,

$$
\begin{aligned}
\frac{L_{f} V_{\alpha}\left(\delta_{\lambda}^{r(\alpha)}(\tilde{x})\right)}{\lambda^{\ell+k}} & =\frac{\partial V_{\alpha}}{\partial \tilde{q}_{v}}\left(\delta_{\lambda}^{r(\alpha)}(\tilde{x})\right) \frac{f_{1}\left(\lambda \tilde{q}_{v}, \lambda^{\alpha} \tilde{\omega}\right)}{\lambda^{\ell+k}}+\frac{\partial V_{\alpha}}{\partial \tilde{\omega}}\left(\delta_{\lambda}^{r(\alpha)}(\tilde{x})\right) \frac{f_{2}\left(\lambda^{\alpha} \tilde{\omega}, \omega_{b I}\right)}{\lambda^{\ell+k}} \\
& =\lambda^{\ell-1} \frac{\partial V_{\alpha}}{\partial \tilde{q}_{v}}(\tilde{x}) \frac{f_{1}\left(\lambda \tilde{q}_{v}, \lambda^{\alpha} \tilde{\omega}\right)}{\lambda^{\ell+k}}+\lambda^{\ell-\alpha} \frac{\partial V_{\alpha}}{\partial \tilde{\omega}}(\tilde{x}) \frac{f_{2}\left(\lambda^{\alpha} \tilde{\omega}, \omega_{b I}\right)}{\lambda^{\ell+k}} \\
& =\frac{\partial V_{\alpha}}{\partial \tilde{q}_{v}}(\tilde{x}) \frac{f_{1}\left(\lambda \tilde{q}_{v}, \lambda^{\alpha} \tilde{\omega}\right)}{\lambda^{\alpha}}+\frac{\partial V_{\alpha}}{\partial \tilde{\omega}}(\tilde{x}) \frac{f_{2}\left(\lambda^{\alpha} \tilde{\omega}, \omega_{b I}\right)}{\lambda^{2 \alpha-1}}
\end{aligned}
$$

where Proposition 3 and the fact that $k=\alpha-1$ have been used. Since $\partial V_{\alpha} / \partial \tilde{q}_{v}$ and $\partial V_{\alpha} / \partial \tilde{\omega}$ are continuous, they are uniformly bounded on $\mathbb{S}^{5}$. Hence, only the terms $f_{1}\left(\lambda \tilde{q}_{v} \lambda^{\alpha} \tilde{\omega}\right) / \lambda^{\alpha}$ and $f_{2}\left(\lambda^{\alpha} \tilde{\omega}, \omega_{b I}\right) / \lambda^{2 \alpha-1}$, need to be considered. The term $f_{1}$ is examined first. From (3.54), $f_{1}\left(\lambda \tilde{q}_{v}, \lambda^{\alpha} \tilde{\omega}\right)=\frac{1}{2}\left(A\left(\lambda \tilde{q}_{v}\right)-I\right) \lambda^{\alpha} \tilde{\omega}$. For $\left\|\tilde{q}_{v}\right\|=1$ (which can occur when $\tilde{x} \in \mathbb{S}^{5}$ ), this only makes sense when $\lambda<1$. Hence, the search for $\lambda_{0}>0$ is restricted such that $\lambda_{0}<1$. Now, applying Proposition 7, for all $\tilde{x} \in \mathbb{S}^{5}$, and using the fact that $\left\|\tilde{q}_{v}\right\|,\|\tilde{\omega}\| \leq\|\tilde{x}\|=1$ yields $\left\|f_{1}\left(\lambda \tilde{q}_{v}, \lambda^{\alpha} \tilde{\omega}\right)\right\| / \lambda^{\alpha} \leq$ $\lambda(1+\lambda) / 2$. Clearly, $f_{1}\left(\lambda \tilde{q}_{v} \lambda^{\alpha} \tilde{\omega}\right) / \lambda^{\alpha} \rightarrow 0$ as $\lambda \downarrow 0$, uniformly on $\mathbb{S}^{5}$.

Next, we examine $f_{2} . \quad$ From (3.54), $\left\|f_{2}\left(\tilde{\omega}, \omega_{b I}\right)\right\| \leq$ $\left\|J^{-1}\right\|\|J\|\left(\|\tilde{\omega}\|\left(2\left\|\omega_{b I}\right\|+\|\tilde{\omega}\|\right)\right)$. Now, $\omega_{b I}$ is time-varying, so an additional assumption is needed in order to be able to proceed. Assume that $\|\omega\|$ is bounded by some $\bar{\omega}$. This then implies that $\left\|\omega_{b I}\right\| \leq \bar{\omega}+\omega_{0}$. Consequently,

$$
\frac{\left\|f_{2}\left(\lambda^{\alpha} \tilde{\omega}, \omega_{b I}\right)\right\|}{\lambda^{2 \alpha-1}} \leq \lambda^{1-\alpha}\left\|J^{-1}\right\|\|J\|\left(2\left(\bar{\omega}+\omega_{0}\right)+\lambda^{\alpha}\right)
$$

where the fact that $\|\tilde{\omega}\| \leq\|\tilde{x}\|=1$ has been used. It is now clear that $f_{2}\left(\lambda^{\alpha} \tilde{\omega}, \omega_{b I}\right) / \lambda^{2 \alpha-1} \rightarrow 0$ as $\lambda \downarrow 0$, uniformly on $\mathrm{S}^{5}$, provided $\|\omega\| \leq \bar{\omega}$.

Based on the above analysis, one can conclude that it is indeed possible to find $\lambda_{0}(\bar{\omega})>0$, such that (3.64) holds for all $\tilde{x} \in \mathbb{S}^{5}$, when $0<\lambda<\lambda_{0}(\bar{\omega})$. It is important to note that $\lambda_{0}(\bar{\omega})$ is a function of the bound $\bar{\omega}$, and consequently, so is the set $U$, 
given in (3.65). In fact, from (3.71) it can be seen that $\lambda_{0}(\bar{\omega})$ decreases (and consequently $U(\bar{\omega})$ shrinks) as $\bar{\omega}$ increases. To show the dependence of $U$ on $\bar{\omega}$, from this point on it shall be denoted by $U(\bar{\omega})$.

While for a given bound $\bar{\omega}$, it has been established that (3.68) holds on the open set $U(\bar{\omega})$, it will be useful to find an open sublevel set of $V_{\alpha}$, contained in $U(\bar{\omega})$, since this will then become an invariant set, and in the absence of the disturbance $\tau_{d}$, will be inside the domain of attraction of the observer. To this end, since $U(\bar{\omega})$ is open, let $\eta>0$ be the radius of a closed ball contained inside $U(\bar{\omega})$. Let $\bar{\kappa}=\min _{\tilde{x} \in \eta S^{5}} V_{\alpha}(\tilde{x})>$ 0 . The set

$$
W=\left\{\tilde{x} \in B_{\eta}(0): V_{\alpha}(\tilde{x})<\bar{\kappa}\right\}
$$

is such a set.

Finally the term $\tilde{d}$ in (3.53) is handled. For any $\tilde{x} \in \mathbb{S}^{5}$, one has from (3.54) that

$$
\begin{aligned}
L_{\tilde{d}} V_{\alpha}\left(\delta_{\lambda}^{r(\alpha)}(\tilde{x})\right) & =\frac{\partial V_{\alpha}}{\partial \tilde{\omega}}\left(\delta_{\lambda}^{r(\alpha)}(\tilde{x})\right) J^{-1} \tau_{d}, \\
& =\lambda^{\ell-\alpha} \frac{\partial V_{\alpha}}{\partial \tilde{\omega}}(\tilde{x}) J^{-1} \tau_{d}, \\
& \leq \lambda^{\ell-\alpha}\left\|\frac{\partial V_{\alpha}}{\partial \tilde{\omega}}(\tilde{x})\right\|\left\|J^{-1}\right\|\left\|\tau_{d}\right\|,
\end{aligned}
$$

where Proposition 3 has been used. Assume that the external disturbance torque is uniformly bounded by $\left\|\tau_{d}\right\| \leq \bar{\tau}_{d}$, and define

$$
a=\max _{\tilde{x} \in \mathbb{S}^{5}}\left\|\frac{\partial V_{\alpha}}{\partial \tilde{\omega}}(\tilde{x})\right\|\left\|J^{-1}\right\|, \quad b=\min _{\tilde{x} \in \mathbb{S}^{5}} V_{\alpha}^{(\ell-\alpha) / \ell}(\tilde{x})>0
$$

By definition of $b, V_{\alpha}^{(\ell-\alpha) / \ell}(\tilde{x}) / b \geq 1$ for all $\tilde{x} \in \mathbb{S}^{5}$. Using these bounds in (3.73) leads to

$$
L_{\tilde{d}} V_{\alpha}\left(\delta_{\lambda}^{r(\alpha)}(\tilde{x})\right) \leq \frac{a \bar{\tau}_{d}}{b} \lambda^{\ell-\alpha} V_{\alpha}^{(\ell-\alpha) / \ell}(\tilde{x})
$$

for all $\tilde{x} \in \mathbb{S}^{5}$. Using the same homogeneity and continuity arguments as made from equations (3.66) to (3.67), one finds that

$$
L_{\tilde{d}} V_{\alpha}(\tilde{x}) \leq \frac{a \bar{\tau}_{d}}{b} V_{\alpha}^{(\ell-\alpha) / \ell}(\tilde{x})
$$

for all $\tilde{x} \in \mathbb{R}^{6}$. 
Finally, combining (3.68) and (3.74), one finds that when $\|\omega\| \leq \bar{\omega}$, for trajectories inside $U(\bar{\omega})$ we have

$$
\begin{aligned}
\dot{V}_{\alpha}(\tilde{x}(t)) & =L_{g_{\alpha}+f} V_{\alpha}(\tilde{x})+L_{\tilde{d}} V_{\alpha}(\tilde{x}(t)) \\
& \leq-\frac{c(\alpha)}{2} V_{\alpha}^{(\ell+\alpha-1) / \ell}(\tilde{x})+\frac{a \bar{\tau}_{d}}{b} V_{\alpha}^{(\ell-\alpha) / \ell}(\tilde{x}) \\
& =-V_{\alpha}^{(\ell-\alpha) / \ell}(\tilde{x})\left(\frac{c(\alpha)}{2} V_{\alpha}^{(2 \alpha-1) / \ell}(\tilde{x})-\frac{a \bar{\tau}_{d}}{b}\right) .
\end{aligned}
$$

From this, it is seen that $\dot{V}_{\alpha}<0$ when

$$
V_{\alpha}(\tilde{x})>\left(\frac{2 a \bar{\tau}_{d}}{c(\alpha) b}\right)^{\ell /(2 \alpha-1)} .
$$

Consequently, to guarantee feasibility for (3.76), the following restriction is made (compare to (3.30))

$$
\left(\frac{2 a \bar{\tau}_{d}}{c(\alpha) b}\right)^{\ell /(2 \alpha-1)}<\bar{\kappa}
$$

This is clearly satisfied if $\bar{\tau}_{d}$ is small enough.

The following result is now obtained:

Lemma 3.5. Choose $\alpha \in(\bar{\alpha}, 1)$. Suppose that $\|\omega(t)\| \leq \bar{\omega}$ and $\left\|\tau_{d}(t)\right\| \leq \bar{\tau}_{d}$ for all $t \in$ $[0, \bar{t})$, for some $\bar{t} \in \mathbb{R}_{+} \bigcup\{\infty\}$, and that (3.77) is satisfied. If $\tilde{x}(0)$ satisfies $V_{\alpha}(\tilde{x}(0))<\bar{\kappa}$, then $V_{\alpha}(\tilde{x}(t))<\bar{\kappa}$ and $\|\tilde{x}(t)\|<\eta$ for all $t \in[0, T)$. If $T=\infty$, then

$$
\limsup _{t \rightarrow \infty} V_{\alpha}(\tilde{x}(t)) \leq\left(\frac{2 a \bar{\tau}_{d}}{c(\alpha) b}\right)^{\ell /(2 \alpha-1)} \text {, and } \limsup _{t \rightarrow \infty}\|\tilde{x}(t)\| \leq \eta_{\ell}<\eta
$$

where

$$
\eta_{\ell}=\max _{\tilde{x} \in F}\|\tilde{x}\|, F=\left\{\tilde{x} \in U(\bar{\omega}): V_{\alpha}(\tilde{x}) \leq\left(\frac{2 a \bar{\tau}_{d}}{c(\alpha) b}\right)^{\ell /(2 \alpha-1)}\right\} .
$$

Proof. This can be obtained using similar arguments as in the proof of Lemma 3.1, noting that $\tilde{x}(t) \in W \subset B_{\eta}(0)$ for all $t \in[0, \bar{t})$ (see the definition of $W$ in (3.72)), and the fact that when $\bar{t}=\infty, \tilde{x}(t) \rightarrow F \subset B_{\eta_{\ell}}(0)$ as $t \rightarrow \infty$.

Remark. In the absence of disturbances $\left(\tau_{d} \equiv 0\right)$, the observer in (3.49) and (3.50) is finite-time convergent when $\alpha<1$. As shown in [Du, S. Li, and Qian, 2011] and [A.M. Zou, 2014], finite-time control laws can generally provide convergence in finite-time. In comparison to asymptotic control laws, finite-time control laws 
provide higher precision control performance and better disturbance rejection properties. Anticipating similar properties for finite-time observers, this is the reason why the parameter $\alpha$ has been included in the proposed observer. In the case $\alpha=1$, a similar result to Lemma 4 can be obtained using $\bar{V}(\tilde{x})$ directly (without the need for $V_{\alpha}$ ), and in this case the observer becomes asymptotically convergent in the absence of disturbances. Ultimately, $\alpha$ provides another tuning parameter for the user to improve the closed-loop performance.

\subsection{Main Result}

The main result can now be presented.

Theorem 3.6. Consider the system described by (3.9) and (3.10), together with the observerbased control law in (3.34), (3.37), (3.40), (3.49) and (3.50). Then, provided the disturbance torque $\tau_{d}$ is small enough (as precisely outlined in (3.78) and (3.79)), the control and observer parameters can be chosen such that $\epsilon, \omega, \tilde{\omega}$ are locally ultimately bounded.

Proof. Choose $K$ in (3.23) such that $\bar{A}_{11}-\bar{A}_{12} K$ is Hurwitz. Choose some $v>0$, and obtain the related $\delta>0$ as explained in between equations (3.26) and (3.27). Next, choose $\bar{S}, \bar{d}$ such that (3.30) holds. Now, the set $G=\left\{\left(x_{1}, S\right) \in \mathbb{R}^{6}: V_{1}\left(x_{1}\right)<\right.$ $\left.\lambda_{\min }(P)(\min \{\delta, 1\})^{2},\|S\|<\bar{S}\right\}$ is bounded. It follows that since $x$ is related to $\left[x_{1}, S\right]$ by the invertible linear transformation

$$
x=\underbrace{\left[\begin{array}{cc}
I & 0 \\
-K & I
\end{array}\right]}_{T} \underbrace{\left[\begin{array}{c}
x_{1} \\
S
\end{array}\right]}_{\bar{x}},
$$

that the set $H=\left\{x \in \mathbb{R}^{6}: x=T \bar{x}, \bar{x} \in G\right\}$ is bounded also. Fix $r>\sup _{x \in H}\|x\|$. Then, $H \subset B_{r}(0)$. Now, select $\rho>0, \sigma, \bar{\delta}$ in Lemma 3.4 such that

$$
\frac{\rho+\sqrt{\rho^{2}+4 \sigma \bar{\delta}}}{2 \sigma}<\bar{S}
$$

and obtain the corresponding $\varrho(r)>0$ in Lemma 3.4. Choose $\ell \geq 2$ in (3.57), and choose $\alpha \in(\bar{\alpha}, 1)$, where $\bar{\alpha}$ is given in the paragraph between equations (3.62) and (3.63). Noting that $\|\omega\| \leq\|x\|$, take $\bar{\omega}=r$ in Lemma 3.5, and obtain the set $U(\bar{\omega})$ 
in (3.65). Choose $\eta>0$ as in paragraph before equation (3.72), such that $\eta<\varrho(r)$, and choose a suitable $\bar{\kappa}$ (as also explained in that same paragraph). Noting from (3.22) that $\|d\| \leq\left\|\tau_{d}\right\| / J_{x}$, we now require from (3.30) and (3.77) the bound on the disturbance torque to satisfy $\bar{\tau}_{d}=\min \left\{\bar{d} J_{x}, L\right\}$, where $L>0$ satisfies

$$
\left(\frac{2 a L}{c(\alpha) b}\right)^{\ell /(2 \alpha-1)}<\overline{\mathcal{K}}
$$

Finally, consider the initial conditions such that $x(0) \in H \subset B_{r}(0)$, and $\tilde{\omega}(0)$ such that $V(\tilde{x}(0))<\bar{\kappa}$. Now, suppose that $x(t)$ exits $B_{r}(0)$ after some finite time. By construction of $B_{r}(0), x(t)$ must exit $H$ before it can exit $B_{r}(0)$. By Lemmas 3.1, 3.2 and 3.5, this is impossible. Hence, $x(t) \in B_{r}(0)$ for all $t \geq 0$, and the ultimate bounds in Lemmas 3.1, 3.4 and 3.5 apply. This concludes the proof.

\subsection{Numerical Examples}

This section presents numerical examples to demonstrate the performance of the proposed observer-based controller under some realistic implementation conditions, including sampled measurements with measurement noise, sample and hold control implementation, uncertainties in the spacecraft inertia matrix, aerodynamic and solar pressure disturbance torques and small orbital eccentricities. Several numerical cases are proposed here. In the nominal case, the response of the system without any external disturbances and idealistic conditions are presented, as well as we draw comparison between idealistic response and sampled-data response. Next, we present the response with time-varying external disturbances to show feasibility of the observer-based control in dealing with external disturbances. Lastly, the case where uncertainties and time-vary disturbances are added to the sampled-data numerical simulations to demonstrate the robustness of the observer-based controller. While the following simulations are numerical implementation of the novel control law previously developed, in practice one would need to take account several practical issues and limitations: (1) hardware physical limitations such as control saturation (minimum and maximum values viable), instantaneous response, ramp-up, size, and geometrical limitations, (2) operational limitations such as computational 
processing limitations of the on-board computer, signal delays, and signal interference, (3) un-modelled dynamics such as spacecraft flexible appendages.

The spacecraft model is taken from [Godard, K. Kumar, and A. Zou, 2013]. The spacecraft is in a Keplerian orbit with semi-major axis $a=6878 \mathrm{~km}$, and initial condition at perigee. The spacecraft nominal moments of inertia used in the control and observer design are $J_{x}=0.0020 \mathrm{~kg} \cdot \mathrm{m}^{2}, J_{y}=0.0017 \mathrm{~kg} \cdot \mathrm{m}^{2}$, and $J_{z}=0.0015$ $\mathrm{kg} \cdot \mathrm{m}^{2}$. The aerodynamic and solar pressure torques are given by

$$
\begin{gathered}
\tau_{a}=1.36 A_{f}\left[\begin{array}{c}
1+\sin \left(\omega_{0} t\right)+0.5 \sin \left(2 \omega_{0} t\right) \\
-\left(5 \times 10^{2}\right)\left[4+2 \sin \left(\omega_{0} t\right)+0.5 \sin \left(2 \omega_{0} t\right)\right] \\
-\left[1+2 \sin \left(\omega_{0} t\right)+0.5 \sin \left(2 \omega_{0} t\right)\right]
\end{array}\right], \\
\tau_{s}=S_{f}\left[\begin{array}{c}
\left(2 \times 10^{-5}\right)\left[1-2 \sin \left(\omega_{0} t\right)\right] \\
\left(1 \times 10^{-3}\right) \cos \left(\omega_{0} t\right) \\
-\left(5 \times 10^{-5}\right) \cos \left(\omega_{0} t\right)
\end{array}\right],
\end{gathered}
$$

respectively, where $A_{f}=1.0 \times 10^{-11}$ and $S_{f}=1.70 \times 10^{-6}$ are derived from "worstcase scenarios" for the RyeSat spacecraft model as outlined in [Godard, K. Kumar, and A. Zou, 2013].

TABLE 3.1: Summary of parameters

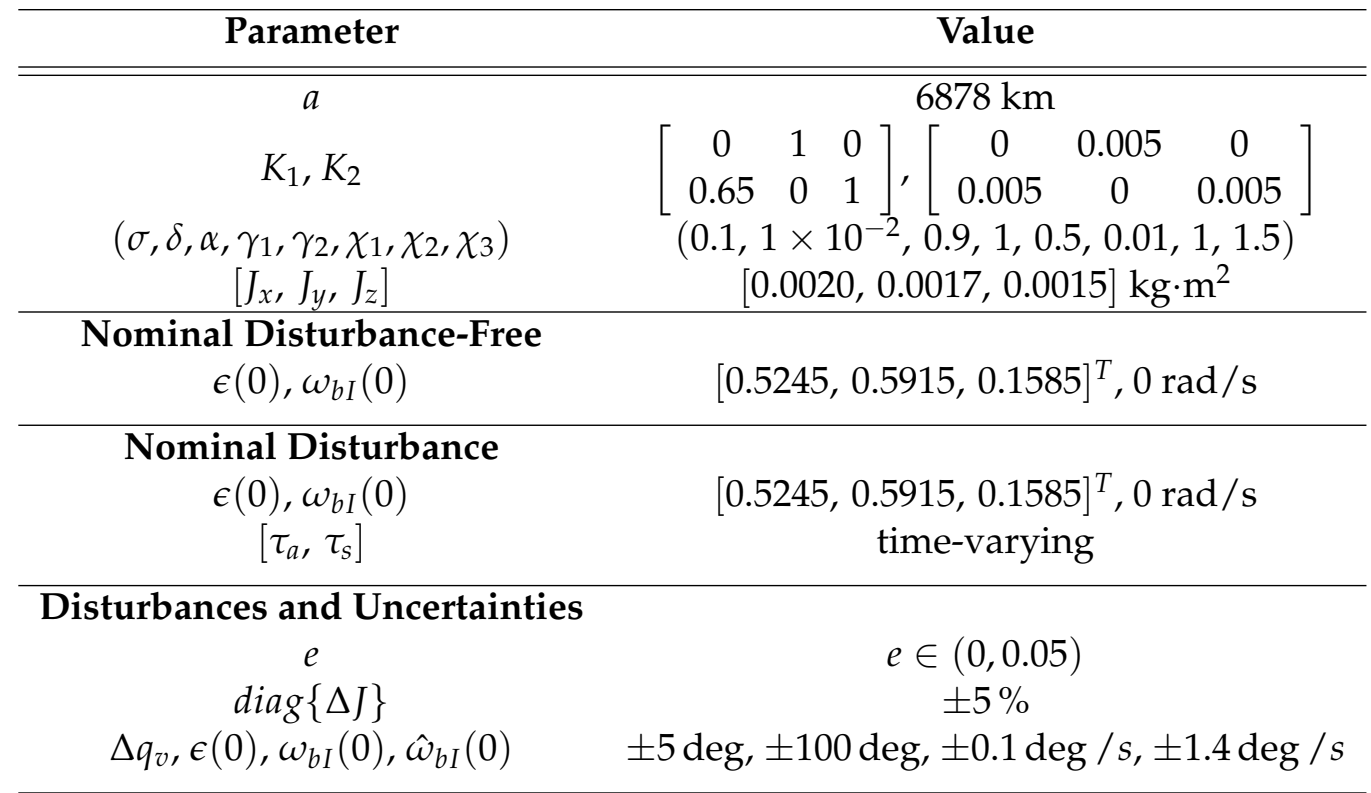


In the numerical examples, attitude measurements are generated at sample times $t_{k}(k=0,1, \ldots)$ according to

$$
\begin{aligned}
& q_{v m}\left(t_{k}\right)=q_{4}\left(t_{k}\right) q_{v e, k}+q_{4 e, k} q_{v}\left(t_{k}\right)+q_{v}\left(t_{k}\right)^{\times} q_{v e, k \prime} \\
& q_{4 m}\left(t_{k}\right)=q_{4}\left(t_{k}\right) q_{4 e, k}-q_{v}\left(t_{k}\right)^{T} q_{v e, k}
\end{aligned}
$$

where $q_{v e, k}$ is generated with MATLAB's "randn" random number generator, with standard deviation of $0.1 \times 180 /(6 \pi)$ in each component and $q_{4 e, k}=\sqrt{1-q_{v e, k}^{T} q_{v e, k}}$. Consequently, $\left(q_{v e, k}, q_{4 e, k}\right)$ is a unit quaternion representing a random rotational measurement error with 0.1 degree error (3-sigma). Using the measured attitude $q_{m}=\left(q_{v m}, q_{4 m}\right)$, the measured rotation matrix from orbital to body coordinates is computed as

$$
C_{b n, m}\left(t_{k}\right)=C\left(q_{m}\left(t_{k}\right)\right) C_{i n}\left(t_{k}\right)
$$

where $C_{i n}$ is the rotation matrix from orbiting to inertial coordinates, which can be obtained from the orbital position and velocity vectors. The corresponding measured attitude error quaternion $\left(\epsilon_{m}\left(t_{k}\right), \eta_{m}\left(t_{k}\right)\right)$ is then extracted from $C_{b n, m}\left(t_{k}\right)$ as [Ruiter, Damaren, and J. Forbes, 2013]

$$
\begin{aligned}
\epsilon_{m}\left(t_{k}\right)^{\times} & =\left(C_{b n, m}^{T}\left(t_{k}\right)-C_{b n, m}\left(t_{k}\right)\right) /\left(4 \eta_{m}\left(t_{k}\right)\right) \\
\eta_{m}\left(t_{k}\right) & =\frac{\left(\operatorname{trace}\left[C_{b n, m}\left(t_{k}\right)\right]+1\right)^{1 / 2}}{2}
\end{aligned}
$$

The sampled-data implementation of the proposed observer-based control law is

$$
\begin{aligned}
\bar{\tau}_{u}(t) & =\Omega^{-1}\left(-\sigma \hat{S}_{m}\left(t_{k}\right)-\hat{\psi}_{m}\left(t_{k}\right)\right), \\
\dot{\hat{q}}(t) & =\frac{1}{2} B(\hat{q}(t)) C^{T}\left(\tilde{q}_{m}(t)\right)\left(\hat{\omega}_{b I}(t)+\gamma_{1} P^{-1}\left(\tilde{q}_{m}(t)\right) \operatorname{sig}^{\alpha}\left(\tilde{q}_{v m}(t)\right)\right), \\
J \dot{\hat{\omega}}_{b I}(t) & =-\hat{\omega}_{b I}(t)^{\times} J \hat{\omega}_{b I}(t)+\tau_{g, m}\left(t_{k}\right)+\tau_{u}(t)+\gamma_{2} J \operatorname{sig}^{\alpha_{1}}\left(\tilde{q}_{v m}(t)\right),
\end{aligned}
$$

then for $t \in\left[t_{k}, t_{k+1}\right)$, where

$$
\begin{gathered}
\hat{S}_{m}\left(t_{k}\right)=K_{1} \hat{\omega}_{m}\left(t_{k}\right)+K_{2} \epsilon_{m}\left(t_{k}\right), \quad \hat{\omega}_{m}\left(t_{k}\right)=\hat{\omega}_{b I}\left(t_{k}\right)+\omega_{0}\left(t_{k}\right) C_{b n, m}\left(t_{k}\right) e_{2}, \\
\hat{\psi}_{m}\left(t_{k}\right)=\hat{\phi}_{m}\left(t_{k}\right) \tanh \left(\frac{2 \hat{\phi}_{m}\left(t_{k}\right) k_{u} \hat{S}_{m}\left(t_{k}\right)}{\bar{\delta}}\right),
\end{gathered}
$$




$$
\hat{\phi}_{m}=\chi_{1}+\chi_{2}\left\|\hat{\omega}_{m}\left(t_{k}\right)\right\|+\chi_{3}\left\|\hat{\omega}_{m}\left(t_{k}\right)\right\|^{2},
$$

and $\tilde{q}_{m}=\left(\tilde{q}_{v m}, \tilde{q}_{4 m}\right)$ is given by

$$
\begin{aligned}
& \tilde{q}_{v m}(t)=-q_{4 m}\left(t_{k}\right) \hat{q}_{v}(t)+\hat{q}_{4}(t) q_{v m}\left(t_{k}\right)-\hat{q}_{v}(t)^{\times} q_{v m}\left(t_{k}\right), \\
& \tilde{q}_{4 m}(t)=q_{4 m}\left(t_{k}\right) \hat{q}_{4}(t)+q_{v m}\left(t_{k}\right)^{T} \hat{q}_{v}(t) .
\end{aligned}
$$

The sample period in this chapter is chosen to be $\Delta T=t_{k+1}-t_{k}=0.1$ seconds as a limited example. Although in practice, the sample period can be at a much slower rate and can have measurement unavailability (drop-outs), and the system response for such frequencies and cases are not presented here.

The numerical integration is performed using a 4th order Runge-Kutta method with step size equal to $\Delta T$. Smaller step sizes were also tested, but did not produce any noticeable change in results.

\subsubsection{Nominal disturbance-free case}

The purpose of the example in this subsection is two-fold: (1) to demonstrate the performance of the observer-based control law under ideal conditions (zero orbital eccentricity, perfect knowledge of the spacecraft inertia matrix, no external disturbances other than gravity-gradient); (2) to compare the performance of the ideal continuous-time (not sampled-data) observer-based control in the absence of measurement noise with the sampled-data observer-based control with noisy measurements. The spacecraft attitude relative to the orbiting frame is given by $\epsilon(0)=[0.5245,0.5915,0.1585]$, while the initial orbital angular velocity is given by $\omega_{b I}(t)=0 \mathrm{rad} / \mathrm{s}$.

Figure 3.2 shows the resulting attitude and angular velocity responses, respectively. It can be seen that the attitude responses are almost identical, attitude convergence is achieved roughly by 1 orbit. While the angular velocity responses are similar, with the sampled-data angular velocity response exhibiting some noise evidently during steady-state, this is due to the presence of noise on the measurements. From this, it can be seen that the sampled-data observer-based control approximates 
the proposed continuous-time control very well. Having established this, the examples presented in the remaining subsections are for the sampled-data controller only.
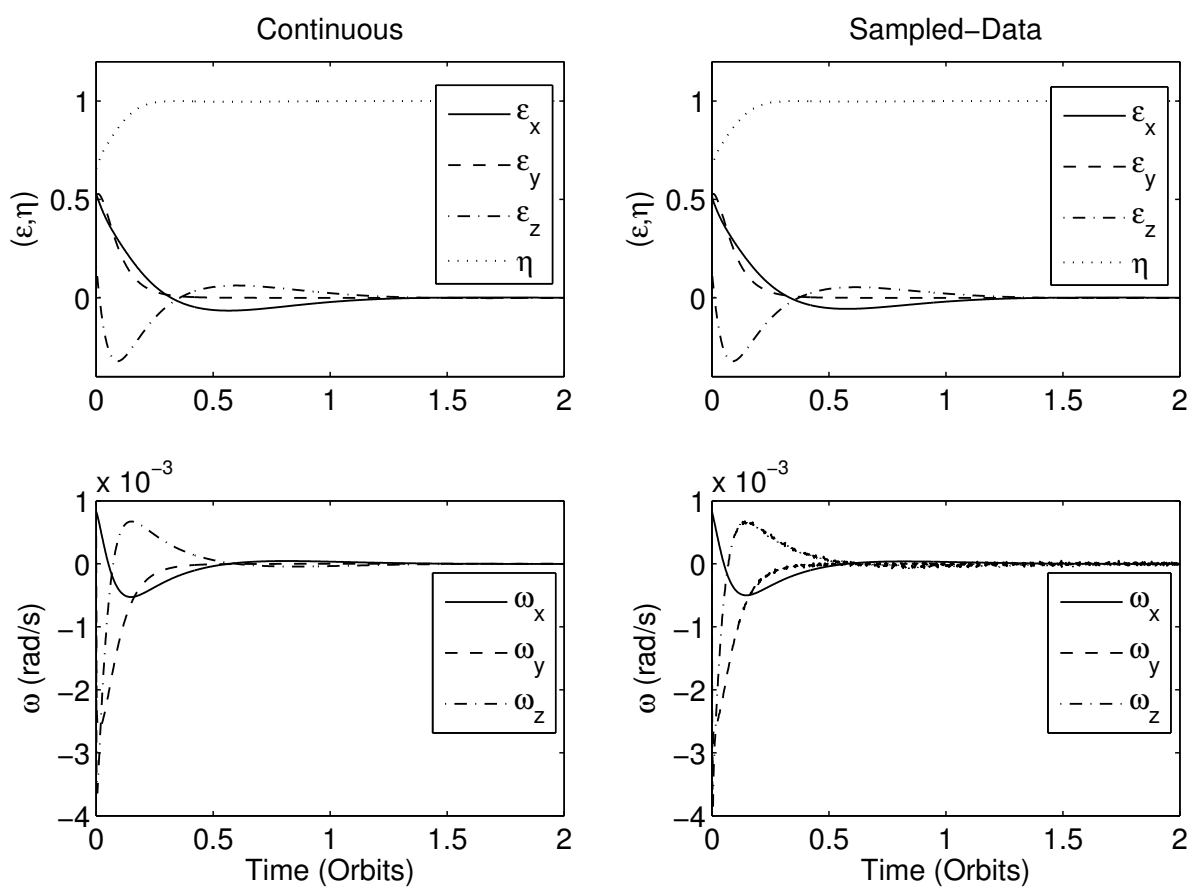

FIGURE 3.2: Attitude and angular velocity responses in nominal disturbance-free case

\subsubsection{Nominal case with disturbances}

The purpose the example in this subsection is to demonstrate the performance of the sampled-data observer-based control law under nominal conditions (zero orbital eccentricity, perfect knowledge of the spacecraft inertia matrix), but with the inclusion of the aerodynamic and solar pressure disturbance torques. Figure 3.3 shows the resulting attitude and angular velocity responses. Figure 3.4 shows the spacecraft control torques. Comparing Figure 3.3 to Figure 3.2, it can be seen that the while the attitude and angular velocity errors still converge to a neighborhood of the origin, the presence of the disturbance torques results in steady-state attitude errors within \pm 0.01 in the quaternion vector and $\pm 10^{-4} \mathrm{rad} / \mathrm{s}$ in the angular velocity, which is to be expected since the disturbance torques act about all three spacecraft axes, while the control torque is limited to the pitch and yaw axes. 

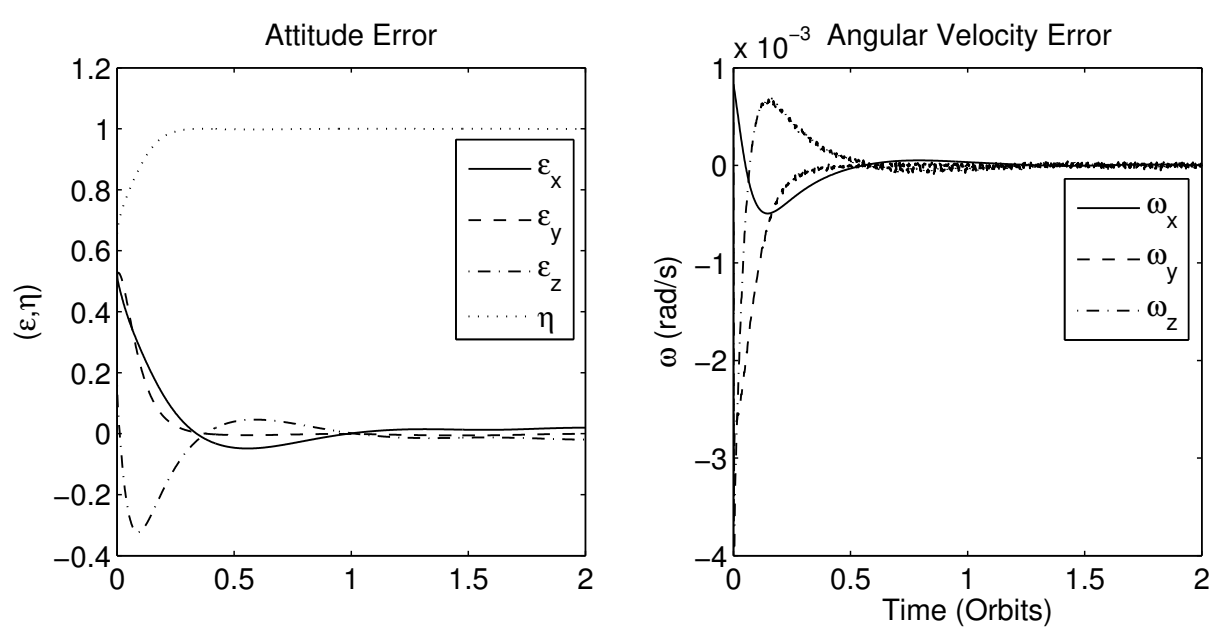

FIGURE 3.3: Attitude and angular velocity responses in nominal case with disturbances
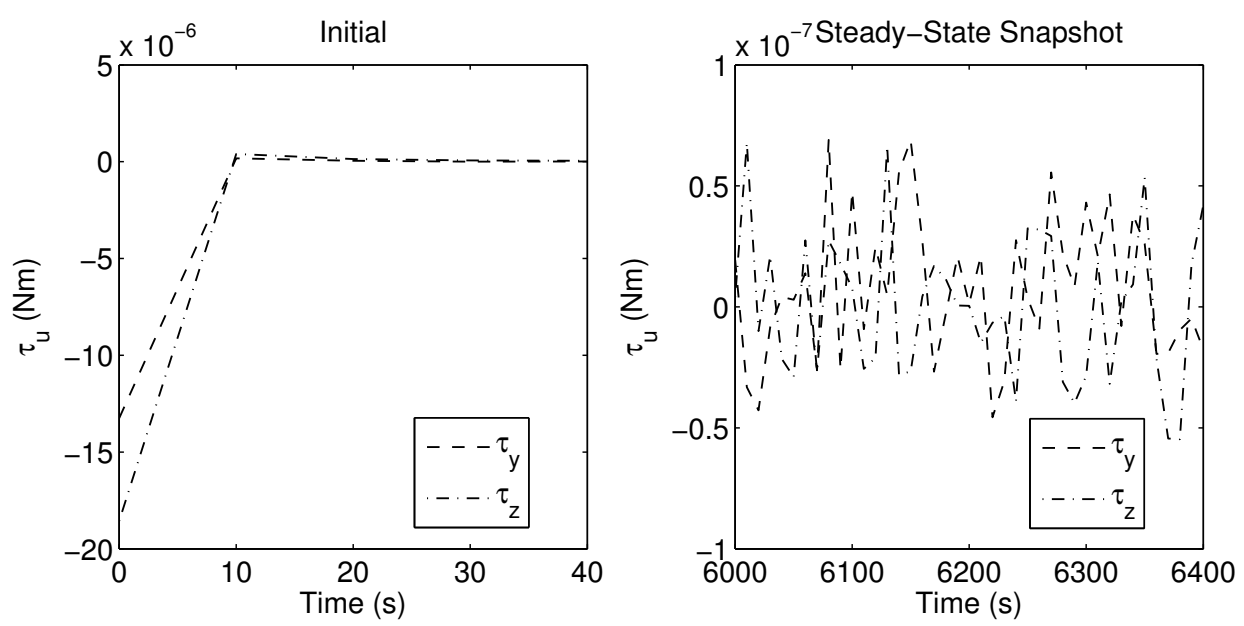

FIGURE 3.4: Control torque in nominal case with disturbances

\subsubsection{All uncertainties and disturbances}

The purpose of the example in this subsection is to demonstrate the robustness of the sampled-data observer-based control law to uncertainties in the spacecraft inertia matrix and non-zero $\left(e_{\max }=0.05\right)$ orbital eccentricities, in the presence of measurement noise for a variety of initial conditions with large initial attitude errors. Furthermore, we conduct test cases for randomized initial conditions, true spacecraft inertia matrix, and orbital eccentricity in a form of fifty Monte-Carlo style simulations. While, this certainly does not represent the probability distribution of the standard error, this simulation is presented to mildly illustrate the efficacy of the controller. 
The true spacecraft inertia matrix is generated as follows. First, the true principal inertia matrix is computed according to

$$
J_{p}=\left[\begin{array}{ccc}
J_{x}+p_{x} & 0 & 0 \\
0 & J_{y}+p_{y} & 0 \\
0 & 0 & J_{z}+p_{z}
\end{array}\right]
$$

where $p_{x}, p_{y}, p_{z}$ are generated using MATLAB's "randn" random number generator, each with standard deviation of $3 \times 10^{-5} \mathrm{~kg} \cdot \mathrm{m}^{2}$. This corresponds to approximately $5 \%$ uncertainty (3-sigma) in the principal inertias. Then, the true spacecraft inertia matrix is obtained as

$$
J_{t}=C\left(q_{J}\right) J_{p} C\left(q_{J}\right)^{T}
$$

where $q_{J}=\left(q_{v J}, q_{4 J}\right), q_{v J}$ is generated with MATLAB's "randn" random number generator, with standard deviation of $5 \times 180 /(6 \pi)$ in each component and $q_{4 J}=$ $\sqrt{1-q_{v J}^{T} q_{v J}}$. Consequently, $q_{J}$ is a unit quaternion representing a 5 degree (3-sigma) random rotation of the principal axes frame. It should be noted that $J_{t}$ is only used in the numerical propagation of the true attitude dynamics. The observer-based controller uses the nominal inertia matrix $J$.

The orbital eccentricity is generated using MATLAB's "rand" function taking values in the interval $e \in(0,0.05)$. It should be noted that the controller is assumed to have access to high quality orbital data, from which the eccentricity can be computed, so the true eccentricity is used by the controller in the computation of the instantaneous orbital angular velocity $\omega_{0}\left(t_{k}\right)$.

The initial true attitude is obtained as $\epsilon(0)=a \sin (\phi / 2), \eta(0)=\cos (\phi / 2)$, where $a \in \mathrm{S}^{2}$ is generated using MATLAB's "randn" function followed by normalization, and $\phi \in \mathbb{R}$ is generated using MATLAB's "randn" function, with standard deviation of $100 \times 180 /(3 \pi)$, corresponding to an initial attitude error of 100 degrees (3-sigma). The initial true inertial angular velocity $\omega_{b I}(0)$ is generated using MATLAB's "randn" function, with standard deviation of $0.1 \times 180 /(3 \pi)$ corresponding to an initial angular velocity of 0.1 degrees/second (3-sigma). The initial estimated inertial angular velocity $\hat{\omega}_{b I}(0)$ is generated using MATLAB's "randn" function, 
with standard deviation of $1.4 \times 180 /(3 \pi)$ corresponding to an initial estimated angular velocity of 1.4 degrees/second (3-sigma). Finally, the initial estimated attitude quaternion $\hat{q}(0)$ is taken as the initial attitude quaternion measurement $q_{m}$ as generated in (3.80).

For clarity in presenting the results of all 50 simulations, the attitude error is presented as the principal angle of rotation corresponding to $\epsilon$, which is given by

$$
\phi=2 \sin ^{-1}(\|\epsilon\|) .
$$

The angular velocity error is presented as $\|\omega\|$. Figure 3.5 shows the resulting attitude and angular velocity responses. It can be seen that in all cases, they converge to neighborhoods of zero. There is even one anomalous case where the attitude error initially grows to 180 degrees, which is due to a high initial angular velocity value, however, the controller still manages to recover the ultimate boundedness. These results demonstrate that even though the theoretical guarantee of ultimate boundedness for the proposed observer-based controller is local and is obtained under nominal conditions where the inertia matrix is perfectly known, the orbit eccentricity is zero, control is continuous and measurements are available without measurement noise, when implemented under realistic conditions in a sampled-data manner the control law retains the ultimate boundedness property even for large initial conditions and dynamic uncertainties.
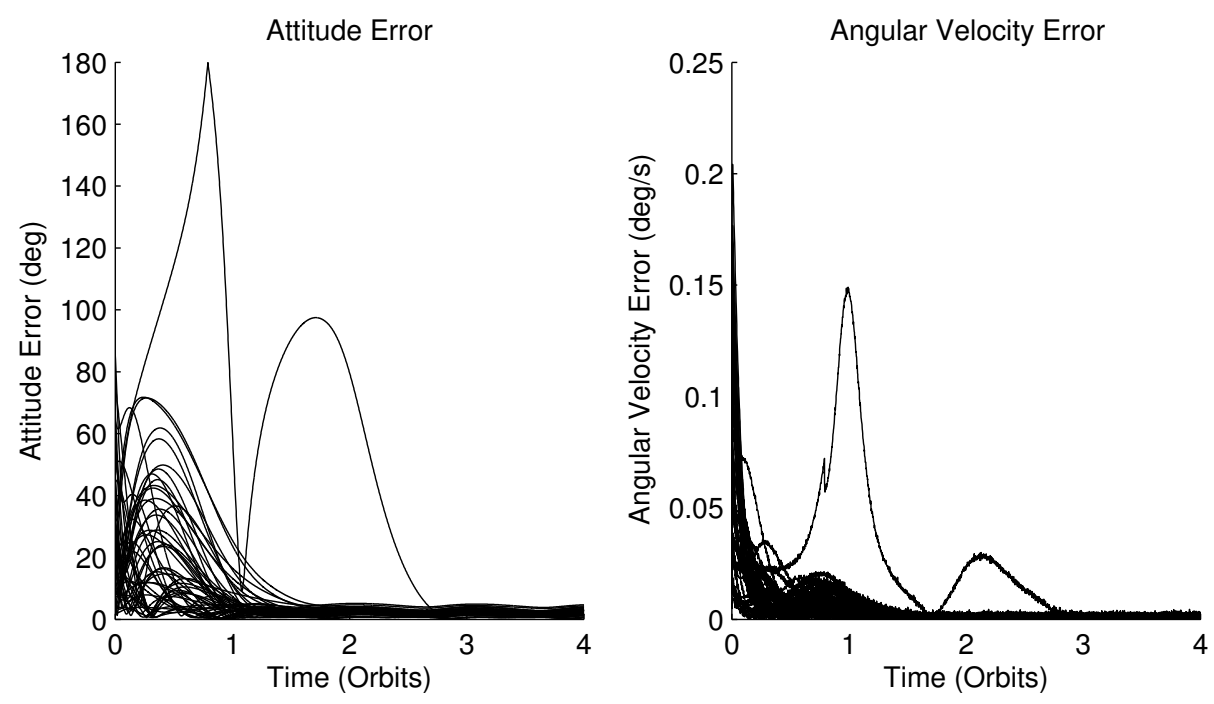

FIGURE 3.5: Attitude and angular velocity responses with inertia matrix uncertainties, non-zero eccentricities and disturbances 


\subsubsection{Effect of $\alpha$ parameter}

In Section 3.3.3, it was mentioned that the observer parameter $\alpha$ provides an additional parameter to tune the closed-loop system performance. This is investigated numerically here, for the sampled-data control implementation with measurement noise, disturbances and system uncertainty. The initial conditions are the same as in subsection 3.5.1, while the true eccentricity and inertia matrix are generated as described in subsection 3.5.3. All control and observer parameters are kept fixed as in Table 3.1, while $\alpha$ takes the values $0.8,0.85,0.9,0.95,1$. Figure 3.6 and 3.7 shows the resulting transient response of various $\alpha$, while Figure 3.8 shows the corresponding steady-state response. While the transient responses are similar across the considered range of $\alpha$, at steady-state (as seen in Figure 3.8), the attitude error initially decreases as $\alpha$ decreases from 1 , but then increases as $\alpha$ becomes smaller. The angular velocity error shows a consistent increase as $\alpha$ decreases from 1 . An explanation for this can be found by considering the innovation terms in the observer, which have the form $\operatorname{sig}^{\alpha}\left(\tilde{q}_{v}\right)$. From its definition, it is clear that $0>\alpha_{1}>\alpha_{2}$ implies $\left|\operatorname{sig}^{\alpha_{1}}(x)\right|>\left|\operatorname{sig}^{\alpha_{2}}(x)\right|$, for any $x \in(-1,1)$. This fact makes the observer more sensitive to measurement noise, once the estimation error becomes small. On the other hand, this fact also makes the observer more robust to system uncertainties and disturbances. The combination of these observations can explain the results observed in Figure 3.8. Namely, when $\alpha$ initially decreases from 1, the effects of system uncertainties and disturbances on the observer are reduced without significantly increasing sensitivity to measurement noise. The result is improved steady-state attitude performance. However, when $\alpha$ is further decreased, then measurement noise becomes the dominant effect, and consequently closed-loop performance deteriorates.

\subsection{Chapter Summary}

This chapter has treated the velocity-free attitude control problem for a nominally nadir-pointing underactuated rigid spacecraft in circular orbits with actuation about only two axes. An observer-based control law has been proposed by combining a 

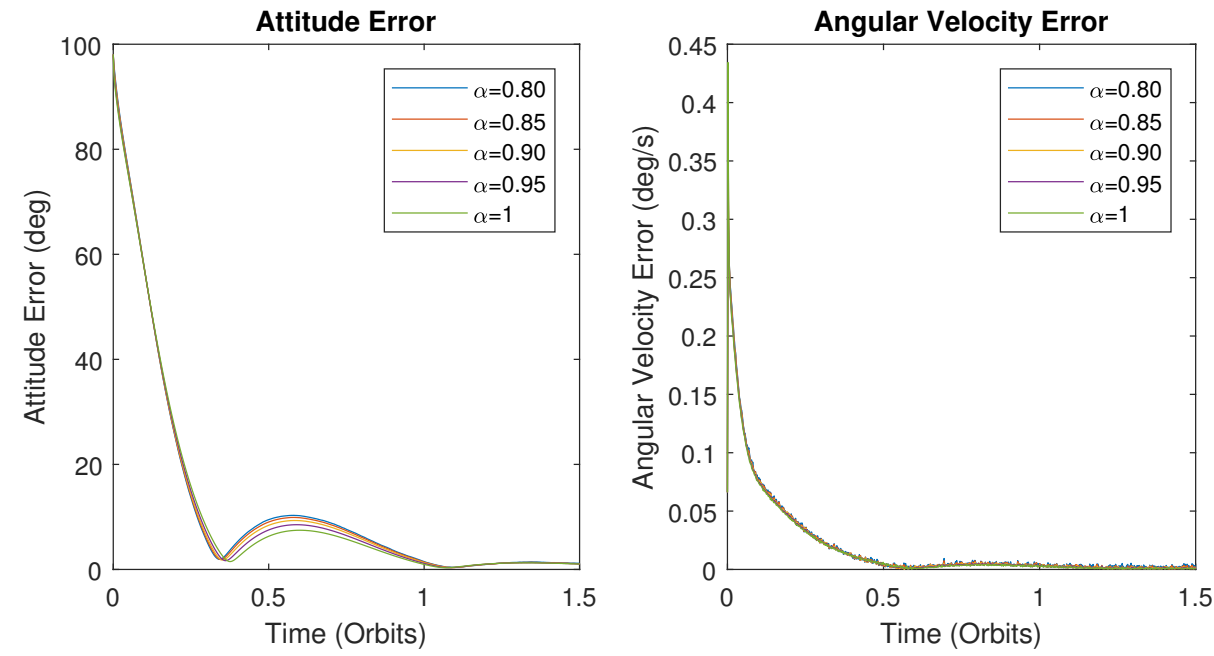

FIGURE 3.6: Effect of $\alpha$ on the transient response

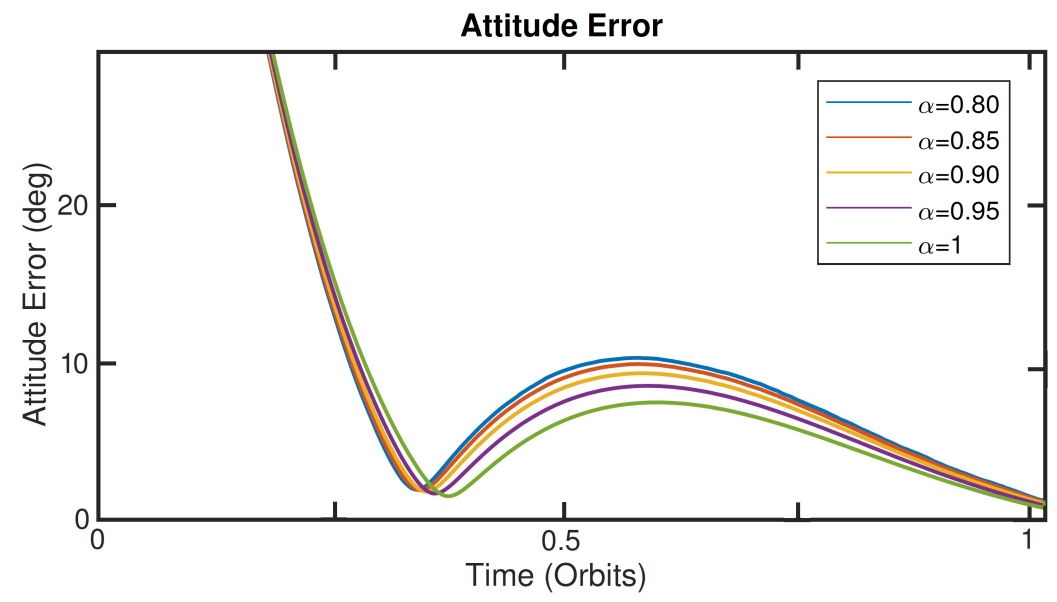

FIGURE 3.7: Effect of $\alpha$ on attitude error
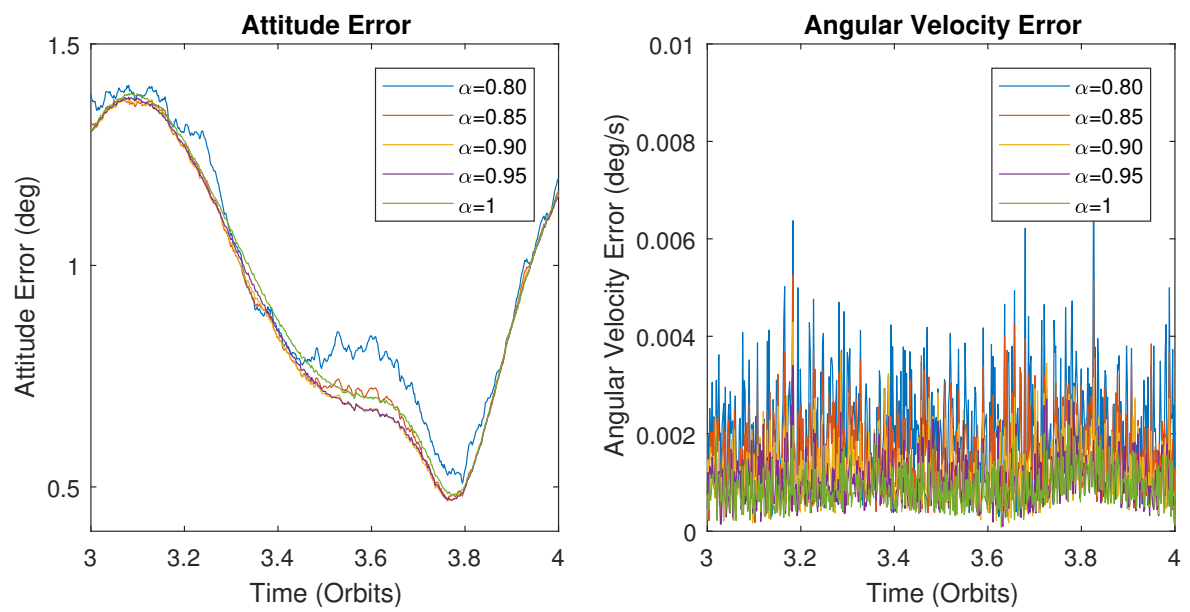

FIGURE 3.8: Effect of $\alpha$ on the steady-state response 
sliding mode control law with an observer to estimate the spacecraft angular velocity. A rigorous analysis has been presented demonstrating that with the proposed observer-based control law, the closed-loop system is locally ultimately bounded. Finally, numerical examples have been presented of a sampled-data implementation of the proposed observer-based control law that demonstrate robustness to uncertainties in the spacecraft inertia matrix, non-zero orbital eccentricity and measurement noise, as well as convergence for large initial attitude errors. 


\section{CHAPTER 4}

\section{Inertial Attitude Stabilization}

\section{using a Generalized Time-Varying Nonlinear Controller}

The problem of regulating the attitude of an inertial pointing underactuated rigid body spacecraft using two torques under time-varying disturbances and inertia matrix uncertainties is examined. A generalized time-varying state feedback controller is proposed that is more versatile in gain tuning, potentially leading to a better performance in comparison to literature. Furthermore, the measurement of angular velocity of the spacecraft is no longer required to achieve asymptotic stability near the origin. The stability of the combined controller-observer system is proven rigorously to show local ultimate boundedness of all signals. Comprehensive numerical examples are shown with sampled-data implementation of the observer-based control law to demonstrate convergence even for very large initial attitude errors.

\subsection{Introduction}

Contrasted to Chapter 3, this chapter utilizes previously developed tools and methodologies to investigate the inertial pointing attitude stabilization of an underactuated rigid body spacecraft using two torques. Since the equations of motion relative to the inertial frame (as opposed to the body-orbital frame in Chapter 3) are highly nonlinear and non-linearizable, for this reason, the previously established observer-based controller is not applicable. Thus, this chapter is motivated by the 
development of a generalized time-varying nonlinear controller for the inertial attitude control problem.

In [A.M. Zou, 2014], the author investigated attitude tracking of a rigid spacecraft through geometric homogeneity and Lyapunov theories. The authors used finite-time time-varying continuous output feedback controller that is based on backstepping technique to achieve semi-global finite-time stability that proved faster convergence rate, better disturbance and parameter perturbation rejection property and higher accuracy control performance than the asymptotic controller. Authors in [Gui, Vukovich, and S. Xu, 2015] investigated spin-axis stabilization of an axisymmetric spacecraft using bounded two torques using a saturated proportional-derivative controller in the uncertainty-free case to produce bounded control torques a priori while ensuring asymptotic stability of closed-loop system. The authors in [Gui, Vukovich, and S. Xu, 2015] designed an adaptive controller based on a sliding-mode-like function to achieve better robustness to inertial uncertainties and disturbances as well as actuator saturation. Similarly, the authors also investigated attitude stabilization of a spacecraft using two parallel control moment gyroscopes using zero momentum and utilized modified direct-inverse (MDI) steering laws by treating internal and external singularities separately [Gui, Vukovich, and S. Xu, 2016].

Utilizing the homogeneity of systems, a velocity-free time-varying continuous feedback controller is formulated to achieve globally exponentially stable with respect to the homogenous norm. The stability of the unactuated closed-loop system and full nominal closed-loop system along with the combined observer-controller system are rigorously proven to provide ultimate boundedness of all signals.

The main features of this chapter is two-fold: 1) a slight generalization of an already existing state-feedback controller for exponential convergence similar to [Morin and Samson, 1997] to achieve inertial attitude stabilization. However, the formulation allows more flexibility with gain selection which can potentially lead to better closed-loop performance and more suitable to implement; 2) the design of an angular velocity observer to remove the necessity of angular velocity measurements 
CHAPTER 4. Inertial Attitude Stabilization using a Generalized Time-Varying Nonlinear Controller

in the control law.

This chapter is organized in the following manner. The problem formulation is constructed in Section 4.2. Also in Section 4.2, the definition of the control objective, the control design for unactuated and actuated states, the observer design, and main results are presented. The numerical examples are produced in Section 4.4 to show effectiveness of the control-observer system for inertial attitude stabilization.

\subsection{Problem Formulation}

\subsubsection{Attitude kinematics and dynamics}

The attitude kinematics and dynamics are reproduced here for convenience.

$$
\begin{aligned}
\dot{q} & =\frac{1}{2} B(q) \omega, \\
J \dot{\omega} & =-\omega^{\times} J \omega+\tau_{u}+\tau_{d},
\end{aligned}
$$

where

$$
B(q)=\left[\begin{array}{c}
P(q) \\
-q_{v}^{T}
\end{array}\right], P(q)=q_{4} I+q_{v}^{\times},
$$

$J \in \mathbb{R}^{3 \times 3}$ is the spacecraft inertia matrix, $\tau_{u}$ is the control torque and $\tau_{d}$ is the disturbance torque. We further separate the disturbance torque into the gravity-gradient torque $\tau_{g}$, and other external disturbances $\bar{\tau}_{d}$,

$$
\tau_{d}=\tau_{g}+\bar{\tau}_{d}
$$

where

$$
\tau_{g}=3 \frac{\mu}{\|R\|^{5}}(C(q) R)^{\times} J C(q) R
$$

and $R \in \mathbb{R}^{3}$ is the orbital position vector in inertial coordinates, and

$$
C(q)=\left(q_{4}^{2}-q_{v}^{T} q_{v}\right) I+2 q_{v} q_{v}^{T}-q_{4} q_{v}^{\times}
$$

is the rotation matrix corresponding to $q$. 
A nominal spacecraft inertia matrix $J_{n} \in \mathbb{R}^{3 \times 3}$ is assumed known, such that $J=$ $J_{n}+\Delta J$, where $\Delta J \in \mathbb{R}^{3 \times 3}$ is the inertia matrix uncertainty. The nominal spacecraft inertia matrix is assumed to have the form

$$
J_{n}=\operatorname{diag}\left\{J_{x}, J_{y}, J_{z}\right\}
$$

It is assumed that the spacecraft is only actuated about the roll and pitch axes, such that

$$
\tau_{u}=J_{n} H u, \quad H=\left[\begin{array}{ll}
1 & 0 \\
0 & 1 \\
0 & 0
\end{array}\right],
$$

where $u=\left[u_{x}, u_{y}\right]^{T}$ is the to be designed control input. To accommodate an angular velocity observer, the control input will be partitioned as $u=u_{n}+\Delta u$, where $u_{n}$ is an ideal state-feedback control law, while $\Delta u$ represents the deviation from $u_{n}$ due to the estimation error of the angular velocity.

\subsubsection{Control objective}

The control objective in this chapter is to regulate the states $\left(q_{v}, \omega\right)$ to a neighborhood of the origin, without the use of angular velocity measurements, in the presence of inertia matrix uncertainties and external disturbance torques. Due to the assumption of bounded time-varying disturbances, there is no stable equilibrium for which the control can drive to and due nature of underactuated system, it is more realistic to state that control objective be in the neighbourhood of the origin.

Since a local stability result will be obtained in this chapter, it is assumed that $q_{4}(t) \geq 0$ for all $t \geq 0$ (the attitude error does not exceed 180 degrees in rotation). Since $\left(q_{v}, q_{4}\right)$ and $\left(-q_{v},-q_{4}\right)$ represent the same attitude, one can take (choose the representation $\left(q_{v}, q_{4}\right)$ for which $\left.q_{4}>0\right)$

$$
q_{4}=\sqrt{1-q_{v}^{T} q_{v}}
$$


The kinematics of $q_{v}$ in (4.1) can therefore be written as

$$
\dot{q}_{v}=\frac{1}{2} \omega+q_{v}^{\times} \omega+\frac{1}{2}\left(\sqrt{1-q_{v}^{T} q_{v}}-1\right) \omega .
$$

The attitude dynamics in (4.2) can now be rewritten as

$$
\dot{\omega}=-J_{n}^{-1} \omega^{\times} J_{n} \omega+H u_{n}+\Omega+J_{n}^{-1} \tau_{d}
$$

where

$$
\Omega=J_{n}^{-1} \omega^{\times} J_{n} \omega-J^{-1} \omega^{\times} J \omega+\left(J^{-1}-J_{n}^{-1}\right) J_{n} H u_{n}+J^{-1} J_{n} H \Delta u+\left(J^{-1}-J_{n}^{-1}\right) \tau_{d} .
$$

Define the unactuated state vector $\eta=\left[q_{1}, q_{2}, q_{3}, \omega_{z}\right]^{T}$, the actuated state vector $y=$ $\left[\omega_{x}, \omega_{y}\right]^{T}$, and the full state vector $x=\left[\eta^{T}, y^{T}\right]^{T}$. The equations of motion in (4.7) and (4.8) can be written as

$$
\underbrace{\left[\begin{array}{c}
\dot{\eta} \\
\dot{y}
\end{array}\right]}_{\dot{x}}=\underbrace{\left[\begin{array}{c}
f_{\eta}(\eta, y) \\
u_{n}
\end{array}\right]}_{f\left(x, u_{n}\right)}+g(x)+h\left(x, t, \tau_{d}, \Delta J, \tilde{\omega}\right)+D,
$$

where

$$
\begin{aligned}
& f_{\eta}(\eta, y)=\left[\begin{array}{c}
\omega_{x} / 2 \\
\omega_{y} / 2 \\
\left(\omega_{z}+q_{1} \omega_{y}-q_{2} \omega_{x}\right) / 2 \\
c_{z} \omega_{x} \omega_{y}
\end{array}\right] \\
& g(x)=\left[\begin{array}{c}
\left(q_{2} \omega_{z}-q_{3} \omega_{y}+\left(\sqrt{1-q_{v}^{T} q_{v}}-1\right) \omega_{x}\right) / 2 \\
\left(q_{3} \omega_{x}-q_{1} \omega_{z}+\left(\sqrt{1-q_{v}^{T} q_{v}}-1\right) \omega_{y}\right) / 2 \\
\left(\sqrt{1-q_{v}^{T} q_{v}}-1\right) \omega_{z} / 2 \\
0 \\
c_{x} \omega_{y} \omega_{z} \\
c_{y} \omega_{x} \omega_{z}
\end{array}\right],
\end{aligned}
$$

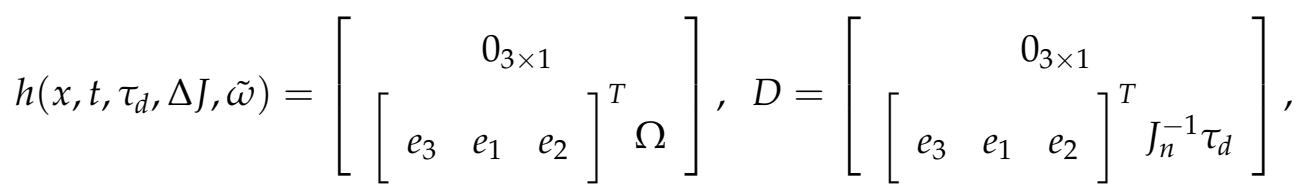


where $c_{x}=\left(J_{y}-J_{z}\right) / J_{x}, c_{y}=\left(J_{z}-J_{x}\right) / J_{y}, c_{z}=\left(J_{x}-J_{y}\right) / J_{z}$, and in the definition of $h\left(x, t, \tau_{d}, \Delta J, \tilde{\omega}\right)$, we recognize in advance that we will design a continuous nominal state-feedback control law $u_{n}(x, t)$, such that when implemented with the estimated angular velocity $\hat{\omega}$, the control deviation will be a continuous function $\Delta u(x, t, \tilde{\omega})$, where $\tilde{\omega}=\omega-\hat{\omega}$ is the angular velocity estimation error.

Assumption 4.1. It is assumed that $c_{z} \neq 0$, otherwise $\omega_{z}$ becomes uncontrollable for the nominal dynamics.

Assumption 4.2. There exist $\tau_{d m}, \bar{\tau}_{d m}>0$ such that $\left\|\tau_{d}(t)\right\| \leq \tau_{d m}$ and $\left\|\bar{\tau}_{d}(t)\right\| \leq \bar{\tau}_{d m}$ for all $t \geq 0$.

Defining the dilation $\delta_{\lambda}^{r}(x)=\left(\lambda q_{1}, \lambda q_{2}, \lambda^{2} q_{3}, \lambda^{2} \omega_{z}, \lambda \omega_{x}, \lambda \omega_{y}\right)$, it is straightforward to verify that the nominal vector field $f\left(x, u_{n}\right)$ is homogeneous of degree zero with respect to $\delta_{\lambda}^{r}$ provided that the state-feedback control inputs $u_{n x}(x, t)$ and $u_{n y}(x, t)$ are homogeneous of degree 1 . Related to the dilation $\delta_{\lambda}^{r}$, we define the homogeneous norm

$$
\rho(x)=\left(q_{1}^{4}+q_{2}^{4}+q_{3}^{2}+\omega_{z}^{2}+\omega_{x}^{4}+\omega_{y}^{4}\right)^{1 / 4} .
$$

\subsubsection{Control design for unactuated states}

We first consider the nominal dynamics for the unactuated states $\eta$, given by

$$
\dot{\eta}=f_{\eta}(\eta, y)
$$

where we treat the actuated states $y$ as the control input. We define a reduced dilation and a reduced homogeneous norm for the unactuated states as $\delta_{\lambda}^{\bar{r}}(\eta)=$ $\left(\lambda q_{1}, \lambda q_{2}, \lambda^{2} q_{3}, \lambda^{2} \omega_{z}\right)$ and

$$
\bar{\rho}(\eta)=\left(q_{1}^{4}+q_{2}^{4}+q_{3}^{2}+\omega_{z}^{2}\right)^{1 / 4},
$$

respectively.

Remark. $\delta_{\lambda}^{\bar{r}}(\eta)$ is compatible with $\delta_{\lambda}^{r}(x)$. Since $f_{\eta}(\eta, y)$ is homogeneous of degree zero with respect to $\delta_{\lambda}^{r}$, if we design a control law $y=v(\eta, t)$, where both components $v_{x}$ and $v_{y}$ are homogeneous of degree 1 with respect to $\delta_{\lambda}^{\bar{r}}$, the resulting 
closed-loop vector field $f_{\eta}(\eta, v(\eta, t))$ becomes homogeneous of degree zero with respect to $\delta_{\lambda}^{\bar{r}}$.

Consider the control law

$$
\begin{aligned}
& v_{x}(\eta, t)=-k_{1} q_{1}-k_{3} \bar{\rho}(\eta) \sin (t / \epsilon), \\
& v_{y}(\eta, t)=-k_{2} q_{2}+\vartheta(\eta) \sin (t / \epsilon),
\end{aligned}
$$

where

$$
\vartheta(\eta)= \begin{cases}\left(k_{4} q_{3}+k_{5} \omega_{z}\right) / \bar{\rho}(\eta), & \eta \neq 0 \\ 0, & \eta=0\end{cases}
$$

and $k_{1}, k_{2}>0, k_{3}, k_{4}, k_{5}$ satisfy $c_{z} k_{3} k_{4}, c_{z} k_{3} k_{5}>0$, and $\epsilon>0$. It is straightforward to verify that both $v_{x}$ and $v_{y}$ are homogeneous of degree 1 with respect to $\delta_{\lambda}^{\bar{r}}$.

Remark. The control law in (4.13) is a generalization of the one presented in [Morin and Samson, 1997] and [H. Li, Yan, and Shi, 2017], in which $k_{3}=k_{4}=k_{5}=1$. Treating these as variables allows the user a greater ability to tune the closed-loop performance.

Proposition 12. $\vartheta(\eta)$ is continuous on $\mathbb{R}^{4}$.

Proof. Since $\bar{\rho}(\eta)$ is positive-definite and continuous on $\mathbb{R}^{4}$, it is clear that $\vartheta(\eta)$ is continuous on $\mathbb{R}^{4} \backslash\{0\}$. Thus, we just need to consider $\eta=0$. First, note that $\left|k_{4} q_{3}+k_{5} \omega_{z}\right| \leq a\left(\left|q_{3}\right|+\left|\omega_{z}\right|\right) \leq \sqrt{2} a\left(q_{3}^{2}+\omega_{z}^{2}\right)^{1 / 2}$, where $a=\max \left\{\left|k_{4}\right|,\left|k_{5}\right|\right\}$. Next, $\left(q_{3}^{2}+\omega_{z}^{2}\right)^{1 / 2} /\left(q_{3}^{2}+\omega_{z}^{2}\right)^{1 / 4}=\left(q_{3}^{2}+\omega_{z}^{2}\right)^{1 / 4} \rightarrow 0$ as $\left(q_{3}, \omega_{z}\right) \rightarrow 0$. Consequently, given $\epsilon>0$, there exists $\delta>0$ such that $\left\|\left(q_{3}, \omega_{z}\right)\right\|<\delta$ implies that $\left(q_{3}^{2}+\omega_{z}^{2}\right)^{1 / 2}<$ $(\epsilon /(\sqrt{2} a))\left(q_{3}^{2}+\omega_{z}^{2}\right)^{1 / 4}$. As a result, $\|\eta\|<\delta$ implies that $\left\|\left(q_{3}, \omega_{z}\right)\right\|<\delta$, which leads to

$$
\left|k_{4} q_{3}+k_{5} \omega_{z}\right| \leq \sqrt{2} a\left(q_{3}^{2}+\omega_{z}^{2}\right)^{1 / 2}<\epsilon\left(q_{3}^{2}+\omega_{z}^{2}\right)^{1 / 4} \leq \epsilon \bar{\rho}(\eta)
$$

Thus, $\lim _{\eta \rightarrow 0} \vartheta(\eta)=0$, and $\vartheta$ is continuous at $\eta=0$ also. This concludes the proof. 
Taking $y=v(\eta, t)$ in (4.13) and substituting into (4.11) gives the nominal unactuated closed-loop dynamics

$$
\dot{\eta}=f_{\eta}(\eta, v(\eta, t))=f_{0}(\eta)+f_{1}(\eta) \sin (t / \epsilon)+f_{2}(\eta) \cos (2 t / \epsilon),
$$

where

$$
\begin{gathered}
f_{0}(\eta)=\left[\begin{array}{c}
-k_{1} q_{1} / 2 \\
-k_{2} q_{2} / 2 \\
\left(\omega_{z}+\left(k_{1}-k_{2}\right) q_{1} q_{2}\right) / 2 \\
c_{z} k_{1} k_{2} q_{1} q_{2}-c_{z} k_{3} k_{4} q_{3} / 2-c_{z} k_{3} k_{5} \omega_{z} / 2
\end{array}\right], \\
f_{1}(\eta)=\left[\begin{array}{c}
-k_{3} \bar{\rho}(\eta) / 2 \\
\vartheta(\eta) / 2 \\
q_{1} \vartheta(\eta) / 2+k_{3} q_{2} \bar{\rho}(\eta) / 2 \\
c_{z} k_{2} k_{3} q_{2} \bar{\rho}(\eta)-c_{z} k_{1} q_{1} \vartheta(\eta)
\end{array}\right], f_{2}(\eta)=\left[\begin{array}{c}
0 \\
0 \\
c_{z}\left(k_{3} k_{4} q_{3}+k_{3} k_{5} \omega_{z}\right) / 2
\end{array}\right] .
\end{gathered}
$$

Unsurprisingly, vector fields $f_{0}(\eta), f_{1}(\eta), f_{2}(\eta)$ are all homogeneous of degree zero with respect to $\delta_{\lambda}^{\bar{r}}$.

We now proceed to construct a homogeneous strict Lyapunov function for (4.15). As in [H. Li, Yan, and Shi, 2017], consider

$$
V(\eta)=a q_{1}^{4}+b q_{2}^{4}+c q_{3}^{2}+d \omega_{z}^{2}+e q_{3} \omega_{z}
$$

where $a, b, c, d, e>0$. It is straightforward to see that $V(\eta)$ is homogeneous of degree 4 with respect to $\delta_{\lambda}^{\bar{r}}$. A necessary and sufficient condition for $V(\eta)$ to be positivedefinite, is that

$$
4 c d>e^{2}
$$

This can be obtained by considering that $V(\eta)$ is positive-definite if and only if $c q_{3}^{2}+$ $d \omega_{z}^{2}+e q_{3} \omega_{z}$ is positive-definite in $\left(q_{3}, \omega_{z}\right)$. In turn, since

$$
c q_{3}^{2}+d \omega_{z}^{2}+e q_{3} \omega_{z}=\left[\begin{array}{ll}
q_{3} & \omega_{z}
\end{array}\right]\left[\begin{array}{cc}
c & e / 2 \\
e / 2 & d
\end{array}\right]\left[\begin{array}{c}
q_{3} \\
\omega_{z}
\end{array}\right]
$$


$c q_{3}^{2}+d \omega_{z}^{2}+e q_{3} \omega_{z}$ is positive-definite if and only if the above matrix is positivedefinite, leading to (4.17).

We now first consider the averaged system corresponding to (4.15) given by the vector field $f_{0}(\eta)$. We find that the Lie derivative of $V$ along $f_{0}$ is given by

$$
\begin{aligned}
L_{f_{0}} V(\eta)= & -2 a k_{1} q_{1}^{4}-2 b k_{2} q_{2}^{4}-\left(e c_{z} k_{3} k_{4} / 2\right) q_{3}^{2}-\left(d c_{z} k_{3} k_{5}-e / 2\right) \omega_{z}^{2} \\
& +\left(c-d c_{z} k_{3} k_{4}-e c_{z} k_{3} k_{5} / 2\right) q_{3} \omega_{z}+\left(c\left(k_{1}-k_{2}\right)+e c_{z} k_{1} k_{2}\right) q_{1} q_{2} q_{3} \\
& +\left(2 d c_{z} k_{1} k_{2}+e\left(k_{1}-k_{2}\right) / 2\right) q_{1} q_{2} \omega_{z} .
\end{aligned}
$$

By Young's inequality, we have

$\left|q_{3} \omega_{z}\right| \leq q_{3}^{2} / 2+\omega_{z}^{2} / 2, \quad\left|q_{1} q_{2} q_{3}\right| \leq q_{1}^{2} q_{2}^{2} /(2 \delta)+\delta q_{3}^{2} / 2, \quad\left|q_{1} q_{2} \omega_{z}\right| \leq q_{1}^{2} q_{2}^{2} /(2 \delta)+\delta \omega_{z}^{2} / 2$,

for any $\delta>0$. Applying Young's inequality again to the second two inequalities leads to

$$
\left|q_{1} q_{2} q_{3}\right| \leq\left(q_{1}^{4}+q_{2}^{4}\right) /(4 \delta)+\delta q_{3}^{2} / 2, \quad\left|q_{1} q_{2} \omega_{z}\right| \leq\left(q_{1}^{4}+q_{2}^{4}\right) /(4 \delta)+\delta \omega_{z}^{2} / 2
$$

Utilizing these inequalities, we can now upper-bound $L_{f_{0}} V(\eta)$ in (4.18) by

$$
\begin{aligned}
L_{f_{0}} V(\eta) \leq & -\left(2 a k_{1}-\left|c\left(k_{1}-k_{2}\right)+e c_{z} k_{1} k_{2}\right| /(4 \delta)\right. \\
& \left.-\left|2 d c_{z} k_{1} k_{2}+e\left(k_{1}-k_{2}\right) / 2\right| /(4 \delta)\right) q_{1}^{4} \\
& -\left(2 b k_{2}-\left|c\left(k_{1}-k_{2}\right)+e c_{z} k_{1} k_{2}\right| /(4 \delta)\right. \\
& \left.-\left|2 d c_{z} k_{1} k_{2}+e\left(k_{1}-k_{2}\right) / 2\right| /(4 \delta)\right) q_{2}^{4} \\
& -\left(e c_{z} k_{3} k_{4} / 2-\delta\left|c\left(k_{1}-k_{2}\right)+e c_{z} k_{1} k_{2}\right| / 2\right. \\
& \left.-\left|c-d c_{z} k_{3} k_{4}-e c_{z} k_{3} k_{5} / 2\right| / 2\right) q_{3}^{2} \\
& -\left(d c_{z} k_{3} k_{5}-e / 2-\delta\left|2 d c_{z} k_{1} k_{2}+e\left(k_{1}-k_{2}\right) / 2\right| / 2\right. \\
& \left.-\left|c-d c_{z} k_{3} k_{4}-e c_{z} k_{3} k_{5} / 2\right| / 2\right) \omega_{z}^{2} .
\end{aligned}
$$


We now proceed to choose $a, b, c, d$, e to make $V(\eta)$ positive-definite and $L_{f_{0}} V(\eta)$ negative definite. First, fix $e>0$. Next, choose $d>0$ such that

$$
d>e /\left(2 c_{z} k_{3} k_{5}\right)
$$

Next, we set

$$
c=d c_{z} k_{3} k_{4}+e c_{z} k_{3} k_{5} / 2
$$

To ensure that an appropriate $d>0$ is chosen, recall that (4.17) must hold. Substituting (4.21) into (4.17) leads to

$$
4 d^{2} c_{z} k_{3} k_{4}+4 d e c_{z} k_{3} k_{5} / 2>e^{2}
$$

Noting that the left-hand sides of (4.20) and (4.22) both tend to positive infinity as $d$ increases, we can choose $d>0$ to simultaneously satisfy (4.20) and (4.22), and then with $c$ given by (4.21), the condition in (4.17) is automatically satisfied. Next, having chosen appropriate $c, d, e$, select $\delta>0$ small enough such that

$$
e c_{z} k_{3} k_{4}>\delta\left|c\left(k_{1}-k_{2}\right)+e c_{z} k_{1} k_{2}\right|, d c_{z} k_{3} k_{5}-e / 2>\delta\left|2 d c_{z} k_{1} k_{2}+e\left(k_{1}-k_{2}\right) / 2\right| / 2 .
$$

With this choice of $c, d, e, \delta$, the coefficients of $q_{3}^{2}, \omega_{z}^{2}$ in (4.19) are negative. Finally choose $a, b>0$ large enough to make the coefficients of $q_{1}^{4}, q_{2}^{4}$ in (4.19) negative. We now summarize our initial findings.

Proposition 13. Consider $f_{0}(\eta)$ as defined in (4.15), with $k_{1}, k_{2}>0$ and $k_{3}, k_{4}, k_{5}$ satisfying $c_{z} k_{3} k_{4}, c_{z} k_{3} k_{5}>0$. Then, there exists a smooth positive-definite $V: \mathbb{R}^{4} \rightarrow \mathbb{R}$ satisfying

$$
\beta_{1} \bar{\rho}^{4}(\eta) \leq V(\eta) \leq \beta_{2} \bar{\rho}^{4}(\eta), L_{f_{0}} V(\eta) \leq-\beta_{3} \bar{\rho}^{4}(\eta)
$$

for all $\eta \in \mathbb{R}^{4}$, for some $\beta_{1}, \beta_{2}, \beta_{3}>0$.

Proof. Consider $V(\eta)$ as constructed above, which is homogeneous of degree 4 with respect to $\delta_{\lambda}^{\bar{r}}$. From Proposition $4, L_{f_{0}} V(\eta)$ is homogeneous of degree 4 with respect to $\delta_{\lambda}^{\bar{r}}$. Since $V$ is positive-definite, and $L_{f_{0}} V$ is negative definite, the result 
follows from Corollary 1, with $\beta_{1}=\min _{\eta: \bar{\rho}(\eta)=1} V(\eta), \beta_{2}=\max _{\eta: \bar{\rho}(\eta)=1} V(\eta)$ and $\beta_{3}=-\max _{\eta: \bar{\rho}(\eta)=1} L_{f_{0}} V(\eta)$. This concludes the proof.

We now proceed to the full nominal reduced system in (4.15), following the general approach presented in [Morin and Samson, 1997]. The Lie derivative of $V$ along $f_{\eta}$ is given by

$$
L_{f_{\eta}} V(\eta)=L_{f_{0}} V(\eta)+\frac{\partial V}{\partial \eta} f_{1}(\eta) \sin (t / \epsilon)+\frac{\partial V}{\partial \eta} f_{2}(\eta) \cos (2 t / \epsilon) .
$$

The last two terms here require treatment. Consider the function

$$
W(\eta, t)=V(\eta)+\epsilon \tilde{V}(\eta, t / \epsilon),
$$

where

$$
\tilde{V}(\eta, t)=\cos (t) \frac{\partial V}{\partial \eta} f_{1}(\eta)-\frac{1}{2} \sin (2 t) \frac{\partial V}{\partial \eta} f_{2}(\eta) .
$$

Note that $\epsilon \tilde{V}(\eta, t)$ is simply the negative anti-derivative with respect to $t$ of the two terms we wish to eliminate in $L_{f_{\eta}} V(\eta)$.

For $W(\eta, t)$ to be a suitable candidate for a Lyapunov function, it needs to be continuously differentiable. Now, $V(\eta)$ and $f_{2}(\eta)$ are smooth. However, $f_{1}$ is smooth everywhere except at $\eta=0$, so we need to check that $(\partial V / \partial \eta) f_{1}$ is continuously differentiable at $\eta=0$. Since $(\partial / \partial \eta)\left((\partial V / \partial \eta) f_{1}\right)$ is smooth on $\mathbb{R}^{4} \backslash\{0\}$, it is enough to show that $\left(\partial / \partial \eta_{i}\right)\left((\partial V / \partial \eta) f_{1}\right)(\eta) \rightarrow 0$ as $\|\eta\| \rightarrow 0$ for $i=1,2,3,4$. We recognize utilizing Proposition 3 that $\left(\partial / \partial \eta_{i}\right)\left((\partial V / \partial \eta) f_{1}\right)$ is homogeneous of degree 3 for $i=1,2$, and homogeneous of degree 2 for $i=3,4$ on $\mathbb{R}^{4} \backslash\{0\}$. By Rosier [Rosier, 1992], any $\eta \neq 0$ can be written as $\eta=\delta_{\psi_{t}(\eta)}^{\bar{r}}\left(\psi_{y}(\eta)\right)$, where $\psi_{y}(\eta) \in \mathbb{S}^{3}$. Consequently for $\eta \neq 0$,

$$
\begin{aligned}
\left|\frac{\partial}{\partial \eta_{i}}\left(\frac{\partial V}{\partial \eta} f_{i}\right)(\eta)\right| & =\left|\frac{\partial}{\partial \eta_{i}}\left(\frac{\partial V}{\partial \eta} f_{i}\right)\left(\delta_{\psi_{t}(\eta)}^{\bar{r}}\left(\psi_{y}(\eta)\right)\right)\right|, \\
& =\psi_{t}^{k}(\eta)\left|\frac{\partial}{\partial \eta_{i}}\left(\frac{\partial V}{\partial \eta} f_{i}\right)\left(\psi_{y}(\eta)\right)\right|, \\
& \leq \psi_{t}^{k}(\eta) p,
\end{aligned}
$$


where $k=3$ for $i=1,2$ and $k=2$ for $i=3,4$, and

$$
p=\max _{\eta \in \mathbb{S}^{3}}\left|\frac{\partial}{\partial \eta_{i}}\left(\frac{\partial V}{\partial \eta} f_{i}\right)\left(\delta_{\psi_{t}(\eta)}^{\bar{r}}\left(\psi_{y}(\eta)\right)\right)\right| .
$$

By Rosier [Rosier, 1992], $\psi_{t}^{k}(\eta) \rightarrow 0$ as $\|\eta\| \rightarrow 0$, and consequently so does $\left(\partial / \partial \eta_{i}\right)\left((\partial V / \partial \eta) f_{1}\right)(\eta)$. We conclude that $W(\eta, t)$ is at least continuously differentiable in all of its arguments.

We shall now find restrictions on $\epsilon>0$ such that $W(\eta, t)$ is a strict Lyapunov function for (4.15). We now evaluate

$$
\frac{\partial W}{\partial t}+\frac{\partial W}{\partial \eta} f_{\eta}(\eta, v(\eta, t))=L_{f_{0}} V(\eta)+\epsilon \frac{\partial \tilde{V}}{\partial \eta} f_{\eta}(\eta, v(\eta, t))
$$

Noting that $\tilde{V}$ and $(\partial \tilde{V} / \partial \eta) f_{\eta}$ are both continuous, periodic in $t$ with period $T=2 \pi$, and homogeneous of degree 4 with respect to $\delta_{\lambda}^{\bar{r}}$, from Corollary 1 we obtain

$$
|\tilde{V}(\eta, t)| \leq \kappa_{1} \bar{\rho}^{4}(\eta),\left|\frac{\partial \tilde{V}}{\partial \eta} f_{\eta}(\eta, v(\eta, t))\right| \leq \kappa_{2} \bar{\rho}^{4}(\eta)
$$

for all $(\eta, t) \in \mathbb{R}^{4} \times \mathbb{R}$, where

$$
\kappa_{1}=\max _{\{(\eta, t): \bar{\rho}(\eta)=1,0 \leq t \leq T\}}|\tilde{V}(\eta, t)|, \quad \kappa_{2}=\max _{\{(\eta, t): \bar{\rho}(\eta)=1,0 \leq t \leq T\}}\left|\frac{\partial \tilde{V}}{\partial \eta} f_{\eta}(\eta, v(\eta, t))\right| .
$$

Consequently, from Proposition 13 we find that

$$
W(\eta, t) \geq\left(\beta_{1}-\epsilon \mathcal{K}_{1}\right) \bar{\rho}^{4}(\eta), \frac{\partial W}{\partial t}(\eta, t)+\frac{\partial W}{\partial \eta} f_{\eta}(\eta, v(\eta, t)) \leq-\left(\beta_{3}-\epsilon \mathcal{K}_{2}\right) \bar{\rho}^{4}(\eta),
$$

for all $(\eta, t) \in \mathbb{R}^{4} \times \mathbb{R}$. From this, it is clear that $W(\eta, t)$ is a strict Lyapunov function for (4.15), provided

$$
0<\epsilon<\min \left\{\beta_{1} / \kappa_{1}, \beta_{3} / \kappa_{3}\right\}
$$

Next, we summarize the results.

Proposition 14. Consider the control law in (4.13), with $k_{1}, k_{2}>0$ and $k_{3}, k_{4}, k_{5}$ satisfying $c_{z} k_{3} k_{4}, c_{z} k_{3} k_{5}>0$. Consider $V(\eta)$ as obtained in Proposition 13, and let $\epsilon$ satisfy (4.27). 
Then, $W(\eta, t)$ as defined in (4.24) is continuously differentiable, and satisfies

$$
\bar{\beta}_{1} \bar{\rho}^{4}(\eta) \leq W(\eta, t) \leq \bar{\beta}_{2} \bar{\rho}^{4}(\eta), \frac{\partial W}{\partial t}(\eta, t)+\frac{\partial W}{\partial \eta} f_{\eta}(\eta, v(\eta, t)) \leq-\bar{\beta}_{3} \bar{\rho}^{4}(\eta),
$$

for all $(\eta, t) \in \mathbb{R}^{4} \times \mathbb{R}$, for some $\bar{\beta}_{1}, \bar{\beta}_{2}, \bar{\beta}_{3}>0$.

Proof. By construction $W(\eta, t)$ is homogeneous of degree 4 with respect to $\delta_{\lambda}^{\bar{r}}$, and $\partial W / \partial t(\eta, t)+(\partial W / \partial \eta) f_{\eta}(\eta, v(\eta, t))$ is also homogeneous of degree 4 by Proposition 4. They are both continuous and periodic with period $T=2 \pi \epsilon$. As shown, when $\epsilon$ satisfies (4.27), $W$ is positive-definite, while $\partial W / \partial t(\eta, t)+$ $(\partial W / \partial \eta) f_{\eta}(\eta, v(\eta, t))$ is negative definite. Corollary 1 then yields the result with $\bar{\beta}_{1}=\min _{\bar{\rho}(\eta)=1,0 \leq t \leq T} W(\eta, t), \bar{\beta}_{2}=\max _{\bar{\rho}(\eta)=1,0 \leq t \leq T} W(\eta, t)$ and

$$
\bar{\beta}_{3}=-\max _{\bar{\rho}(\eta)=1,0 \leq t \leq T} \partial W / \partial t(\eta, t)+(\partial W / \partial \eta) f_{\eta}(\eta, v(\eta, t)) .
$$

This concludes the proof.

Corollary 4.2.1. Under the conditions stated in Proposition 14, the nominal unactuated states closed-loop dynamics in (4.15) are globally exponentially stable with respect to the homogeneous norm $\bar{\rho}$.

Proof. Let $\eta(0)$ be given, and $\eta(t)$ the resulting trajectory of (4.15). By Proposition 14 , along the trajectory $\eta(t)$, the Lyapunov function $W(\eta, t)$ satisfies

$$
\begin{aligned}
\dot{W}(\eta(t), t) & =\frac{\partial W}{\partial t}(\eta(t), t)+\frac{\partial W}{\partial \eta} f_{\eta}(\eta(t), v(\eta(t), t)), \\
& \leq-\bar{\beta}_{3} \bar{\rho}^{4}(\eta(t)) \\
& \leq-\left(\bar{\beta}_{3} / \bar{\beta}_{2}\right) W(\eta(t), t) .
\end{aligned}
$$

By the Comparison Principle [Khalil, 1998], $W(\eta(t), t) \leq W(\eta(0), 0) e^{-\left(\bar{\beta}_{3} / \bar{\beta}_{2}\right) t}$. Applying Proposition 14 again leads to $\bar{\rho}(\eta(t)) \leq\left(\bar{\beta}_{2} / \bar{\beta}_{1}\right)^{1 / 4} \bar{\rho}(\eta(0)) e^{-\left(\bar{\beta}_{3} /\left(4 \bar{\beta}_{2}\right)\right) t}$. This concludes the proof.

\subsubsection{Control design for actuated states}

We now consider the full nominal system corresponding to (4.9), given by the vector field $f\left(x, u_{n}\right)$. In the previous section, we established that if the actuated states 
$y=\left[\omega_{x}, \omega_{y}\right]^{T}$ is treated as a control input to the nominal unactuated state dynamics $f_{\eta}(\eta, y)$, then the nominal unactuated state dynamics are globally exponentially stable with control law $y=v(\eta, t)$. We now wish to design the control law $u_{n}$ for the actuated states such that $y$ becomes close to $v(\eta, t)$. However, to ensure that $f\left(x, u_{n}\right)$ is homogeneous of degree zero with respect to $\delta_{\lambda}^{r}$, we must do so in a way that $u_{n x}(x, t)$ and $u_{n y}(x, t)$ are homogeneous of degree 1 .

Following the methodology in [Morin and Samson, 1997], we choose

$$
\begin{aligned}
& u_{n x}(x, t)=-k_{x}\left(\omega_{x}-v_{x}(\eta, t)\right), \\
& u_{n y}(x, t)=-k_{y}\left(\omega_{y}-v_{y}(\eta, t)\right),
\end{aligned}
$$

where $k_{x}, k_{y}>0$. Since $v_{x}, v_{y}$ are homogeneous of degree 1 with respect to $\delta_{\lambda}^{\bar{r}}$ (which is compatible with $\left.\delta_{\lambda}^{r}\right)$, it is readily seen that $u_{n x}(x, t)$ and $u_{n y}(x, t)$ are homogeneous of degree 1 with respect to $\delta_{\lambda}^{r}$.

Remark. For selection of the gains, we can first consider the averaged reduced closed-loop system corresponding to (4.15) given by

$$
\dot{\eta}=f_{0}(\eta)
$$

Linearizing this yields the following averaged reduced dynamics for $\eta$, given by

$$
\dot{q}_{i}+\left(k_{i} / 2\right) q_{i}=0,
$$

for $i=1,2$, and

$$
\ddot{q}_{3}+\left(c_{z} k_{3} k_{5} / 2\right) \dot{q}_{3}+\left(c_{z} k_{3} k_{4} / 4\right) q_{3}=0 \text {. }
$$

These are standard first and second order linear systems, for which there are a wealth of results. Based on these the gains $k_{1}$ to $k_{5}$ may be designed to yield the desired averaged reduced closed-loop dynamics. Following this, $\epsilon>0$ and $k_{x}, k_{y}$ may be tuned to yield the overall closed-loop performance.

We can now proceed to construct a strict Lyapunov Function for the full nominal closed-loop system described by the vector field $f\left(x, u_{n}\right)$, with control law given by (4.28) and (4.29). Following the approach given in [Morin and Samson, 1997]. In 
the sequel, it is assumed that $k_{1}, k_{2}, k_{3}, k_{4}, k_{5}, \epsilon$ have all been chosen to satisfy the conditions stated in Proposition 14. Define the functions

$$
\begin{aligned}
\phi_{i}\left(\eta, t, \omega_{i}\right) & =\int_{\left.v_{i}(\eta, t)\right)}^{\omega_{i}} s^{3}-v_{i}^{3}(\eta, t) d s, \\
& =\omega_{i}^{4} / 4+3 v_{i}^{4}(\eta, t) / 4-\omega_{i} v_{i}^{3}(\eta, t),
\end{aligned}
$$

for $i=x, y$. As remarked in [Morin and Samson, 1997], $\phi_{i}\left(\eta, t, \omega_{i}\right) \geq 0$ for all $\left(\eta, t, \omega_{i}\right) \in \mathbb{R}^{4} \times \mathbb{R} \times \mathbb{R}$, and $\phi_{i}\left(\eta, t, \omega_{i}\right)=0$ if and only if $\omega_{i}=v_{i}(\eta, t)$. It is also straightforward to see from the second line in (4.30) that $\phi_{i}$ is homogeneous of degree 4 with respect to $\delta_{\lambda}^{r}$. Finally, using a similar approach as was used to establish continuous differentiability of $W(\eta, t)$ in (4.24), it can be shown that $\phi_{i}\left(\eta, t, \omega_{i}\right)$ is also continuously differentiable on $\mathbb{R}^{4} \times \mathbb{R} \times \mathbb{R}$. We now consider

$$
\bar{V}(x, t)=W(\eta, t)+\frac{1}{\sqrt{k_{x}}} \phi_{x}\left(\eta, t, \omega_{x}\right)+\frac{1}{\sqrt{k_{y}}} \phi_{y}\left(\eta, t, \omega_{y}\right) .
$$

Since $W(\eta, t)$ is homogeneous of degree 4 with respect to $\delta_{\lambda}^{\bar{r}}$, which is compatible with $\delta_{\lambda}^{r}$, we find that $\bar{V}(x, t)$ is homogeneous of degree 4 with respect to $\delta_{\lambda}^{r}$. We also know that $\bar{V}(x, t)$ is continuously differentiable, periodic with period $T=2 \pi \epsilon$, and non-negative. To show that it is in fact positive-definite, for any $t \in \mathbb{R}, V(x, t)=0$ implies that $W(\eta, t)=0$ and $\phi_{i}\left(\eta, t, \omega_{i}\right)=0$ for $i=x, y$ (since they are all nonnegative). $W(\eta, t)=0$ implies that $\eta=0$, which implies that $v_{i}(\eta, t)=0$ for $i=x, y$. As remarked above, $\phi_{i}\left(\eta, t, \omega_{i}\right)=0$ then implies that $\omega_{i}=0$ for $i=x, y$. Conversely, it is straightforward to see from its definition that $V(0, t)=0$ for all $t \in \mathbb{R}$. Hence, $V(x, t)$ is positive-definite, and by Corollary 1 we obtain

$$
\tilde{\beta}_{1} \rho^{4}(x) \leq \bar{V}(x, t) \leq \tilde{\beta}_{2} \rho^{4}(x),
$$

where $\tilde{\beta}_{1}=\min _{\rho(x)=1,0 \leq t \leq T} \bar{V}(x, t)>0, \tilde{\beta}_{2}=\max _{\rho(x)=1,0 \leq t \leq T} \bar{V}(x, t)$. 
We now compute

$$
\begin{aligned}
\frac{\partial \bar{V}}{\partial t}+\frac{\partial \bar{V}}{\partial x} f\left(x, u_{n}(x, t)\right)= & \frac{\partial W}{\partial t}+\frac{\partial W}{\partial \eta} f_{\eta}(\eta, v(\eta, t)) \\
& +\frac{1}{\sqrt{k_{x}}} \frac{\partial \phi_{x}}{\partial \omega_{x}} u_{n x}(x, t)+\frac{1}{\sqrt{k_{y}}} \frac{\partial \phi_{y}}{\partial \omega_{y}} u_{n y}(x, t) \\
& +\frac{1}{\sqrt{k_{x}}}\left(\frac{\partial \phi_{x}}{\partial t}+\frac{\partial \phi_{x}}{\partial \eta} f_{\eta}(\eta, y)\right)+\frac{1}{\sqrt{k_{y}}}\left(\frac{\partial \phi_{y}}{\partial t}+\frac{\partial \phi_{y}}{\partial \eta} f_{\eta}(\eta, y)\right) \\
& +\frac{\partial W}{\partial \eta}\left(f_{\eta}(\eta, y)-f_{\eta}(\eta, v(\eta, t))\right) .
\end{aligned}
$$

where we have added and subtracted $(\partial W / \partial \eta) f_{\eta}(\eta, v(\eta, t))$. We now deal with (4.33) line by line. First, by Proposition 14, we have

$$
\frac{\partial W}{\partial t}+\frac{\partial W}{\partial \eta} f_{\eta}(\eta, v(\eta, t)) \leq-\bar{\beta}_{3} \bar{\rho}^{4}(\eta)
$$

Next, by (4.28), (4.29) and (4.30), we have

$$
\frac{\partial \phi_{i}}{\partial \omega_{i}} u_{n i}(x, t)=-k_{i}\left(\omega_{i}^{3}-v_{i}^{3}(\eta, t)\right)\left(\omega_{i}-v_{i}(\eta, t)\right)
$$

for $i=x, y$. It can be readily seen that $\frac{\partial \phi_{i}}{\partial \omega_{i}} u_{n i}(x, t) \leq 0$, and $\frac{\partial \phi_{i}}{\partial \omega_{i}} u_{n i}(x, t)=0$ if and only if $\omega_{i}=v_{i}(\eta, t)$. Furthermore, $\frac{\partial \phi_{i}}{\partial \omega_{i}} u_{n i}(x, t)$ is homogeneous of degree 4 with respect to $\delta_{\lambda}^{r}$. As a consequence, the function

$$
F(x, t)=\bar{\rho}^{4}(\eta)+\left(\left(\omega_{x}^{3}-v_{x}^{3}(\eta, t)\right)\left(\omega_{x}-v_{x}(\eta, t)\right)+\left(\omega_{y}^{3}-v_{y}^{3}(\eta, t)\right)\left(\omega_{y}-v_{y}(\eta, t)\right)\right),
$$

is positive-definite and homogeneous of degree 4 with respect to $\delta_{\lambda}^{r}$. It is also periodic with period $T=2 \pi \epsilon$. Now, define the functions on $\left(\mathbb{R}^{6} \backslash\{0\}\right) \times \mathbb{R}$.

$$
H_{i}(x, t)=\left|\frac{\partial \phi_{i}}{\partial t}+\frac{\partial \phi_{i}}{\partial \eta} f_{\eta}(\eta, y)\right| / F(x, t), \quad i=x, y
$$

These functions are homogeneous of degree zero with respect to $\delta_{\lambda}^{r}$, since the numerator and denominator are both homogeneous of degree 4. Define

$$
\Gamma_{i}=\max _{x \in S^{5}, 0 \leq t \leq T} H_{i}(x, t)>0
$$


for $i=x, y$. Then, any $x \neq 0$ can be written as $\delta_{\psi_{t}(x)}^{r}\left(\psi_{y}(x)\right)$, where $\psi_{y}(x) \in \mathrm{S}^{5}$, and $H_{i}(x, t)=H_{i}\left(\delta_{\psi_{t}(x)}^{r}\left(\psi_{y}(x)\right), t\right)=\psi_{t}^{0}(x) H_{i}\left(\psi_{y}(x), t\right) \leq \Gamma_{i}$. As a result,

$$
\frac{\partial \phi_{i}}{\partial t}+\frac{\partial \phi_{i}}{\partial \eta} f_{\eta}(\eta, y) \leq \Gamma_{i} F(x, t), \quad i=x, y,
$$

for all $(x, t) \in \mathbb{R}^{6} \times \mathbb{R}$ (note that (4.38) can be directly verified for $x=0$ ).

Define the set $X=\left\{(x, t): x \in \mathbb{S}^{5}, 0 \leq t \leq T\right\} \backslash\{(x, t): y=v(x, t)\}$, and consider the following function

$$
G(x, t)=\frac{\left|\frac{\partial W}{\partial \eta}\left(f_{\eta}(\eta, y)-f_{\eta}(\eta, v(\eta, t))\right)\right|-\left(\bar{\beta}_{3} / 2\right) \bar{\rho}^{4}(\eta)}{\left(\omega_{x}^{3}-v_{x}^{3}(\eta, t)\right)\left(\omega_{x}-v_{x}(\eta, t)\right)+\left(\omega_{y}^{3}-v_{y}^{3}(\eta, t)\right)\left(\omega_{y}-v_{y}(\eta, t)\right)} .
$$

Note that $G(x, t)$ is homogeneous of degree zero with respect to $\delta_{\lambda}^{r}$. We now show that $G(x, t)$ is bounded from above on $X$. Suppose to the contrary that it is unbounded. Then, we can find a sequence $\left\{\left(x_{i}, t_{i}\right)\right\} \in X \subset \mathbb{S}^{5} \times[0, T]$, such that $\lim _{i \rightarrow \infty} G\left(x_{i}, t_{i}\right)=\infty$. Since $S^{5} \times[0, T]$ is compact, taking a subsequence if necessary, it follows that $\left\{\left(x_{i}, t_{i}\right)\right\}$ converges to an element $(\bar{x}, \bar{t}) \in \mathbb{S}^{5} \times[0, T]$. By continuity of the numerator of $G(x, t)$ and the compactness of $\mathbb{S}^{5} \times[0, T]$, the numerator is bounded. Hence, the denominator of $G\left(x_{i}, t_{i}\right)$ must converge to zero, that is, $\bar{y}=v(\bar{\eta}, \bar{t})$. Now, since $\bar{x} \in \mathbb{R}^{5}$, it must be that $\bar{\eta} \neq 0$ (since otherwise we would also have $\bar{y}=v(0, t)=0)$. By continuity of $\left|(\partial W / \partial \eta)\left(f_{\eta}(\eta, y)-f_{\eta}(\eta, v(\eta, t))\right)\right|$, this term converges to zero as $i \rightarrow \infty$, and the numerator of $G\left(x_{i}, t_{i}\right)$ becomes negative for large enough $i$. However, this contradicts the original assertion that $\lim _{i \rightarrow \infty} G\left(x_{i}, t_{i}\right)=\infty$. Consequently, $G(x, t)$ is bounded from above on $X$. Taking

$$
\Gamma_{3}=\sup _{(x, t) \in X} G(x, t)
$$

we obtain

$$
\begin{aligned}
\left|\frac{\partial W}{\partial \eta}\left(f_{\eta}(\eta, y)-f_{\eta}(\eta, v(\eta, t))\right)\right| \leq & \left(\bar{\beta}_{3} / 2\right) \bar{\rho}^{4}(\eta) \\
& +\Gamma_{3}\left(\left(\omega_{x}^{3}-v_{x}^{3}(\eta, t)\right)\left(\omega_{x}-v_{x}(\eta, t)\right)\right. \\
& \left.+\left(\omega_{y}^{3}-v_{y}^{3}(\eta, t)\right)\left(\omega_{y}-v_{y}(\eta, t)\right)\right)
\end{aligned}
$$


for all $(x, t) \in \mathbb{R}^{6} \times \mathbb{R}$. Inequality (4.40) can be shown to hold directly for the case $y=v(x, t)$, while for $\left(\mathbb{R}^{6} \times \mathbb{R}\right) \backslash\{(x, t): y=v(x, t)\}$ (4.40) can be shown to hold by applying similar homogeneity arguments as before.

Finally, substituting (4.34), (4.35), (4.38) and (4.40) into (4.33), we obtain

$$
\begin{aligned}
\frac{\partial \bar{V}}{\partial t}+ & \frac{\partial \bar{V}}{\partial x} f\left(x, u_{n}(x, t)\right) \leq-\left(\bar{\beta}_{3} / 2-\Gamma_{x} / \sqrt{k_{x}}-\Gamma_{y} / \sqrt{k_{y}}\right) \bar{\rho}^{4}(\eta) \\
& -\left(\sqrt{k_{x}}-\Gamma_{3}-\Gamma_{x} / \sqrt{k_{x}}-\Gamma_{y} / \sqrt{k_{y}}\right)\left(\omega_{x}^{3}-v_{x}^{3}(\eta, t)\right)\left(\omega_{x}-v_{x}(\eta, t)\right) \\
& -\left(\sqrt{k_{y}}-\Gamma_{3}-\Gamma_{x} / \sqrt{k_{x}}-\Gamma_{y} / \sqrt{k_{y}}\right)\left(\omega_{y}^{3}-v_{y}^{3}(\eta, t)\right)\left(\omega_{y}-v_{y}(\eta, t)\right),
\end{aligned}
$$

for all $(x, t) \in \mathbb{R}^{6} \times \mathbb{R}$. We see that $\partial \bar{V} / t \partial t+(\partial \bar{V} / \partial x) f\left(x, u_{n}(x, t)\right)$ is negative definite if we choose $k_{x}, k_{y}$ such that

$$
\begin{aligned}
0<\bar{\beta}_{3} / 2-\Gamma_{x} / \sqrt{k_{x}}-\Gamma_{y} / \sqrt{k_{y}} \\
0<\sqrt{k_{x}}-\Gamma_{3}-\Gamma_{x} / \sqrt{k_{x}}-\Gamma_{y} / \sqrt{k_{y}} \\
0<\sqrt{k_{y}}-\Gamma_{3}-\Gamma_{x} / \sqrt{k_{x}}-\Gamma_{y} / \sqrt{k_{y}} .
\end{aligned}
$$

These inequalities are clearly feasible for $k_{x}, k_{y}$ (simply make them large enough). Next, the following Proposition is proposed.

Proposition 15. Consider the control law defined in (4.13), (4.28) and (4.29), where $k_{1}, k_{2}$, $k_{3}, k_{4}, k_{5}, \epsilon$ satisfy the conditions stated in Proposition 14, and $k_{x}, k_{y}$ satisfy (4.42). Then, $\bar{V}(x, t)$ as defined in (4.31) is continuously differentiable, and satisfies

$$
\tilde{\beta}_{1} \rho^{4}(x) \leq \bar{V}(x, t) \leq \tilde{\beta}_{2} \rho^{4}(x), \quad \frac{\partial \bar{V}}{\partial t}(x, t)+\frac{\partial \bar{V}}{\partial x} f\left(x, u_{n}(x, t)\right) \leq-\tilde{\beta}_{3} \bar{V}(x, t),
$$

for all $(x, t) \in \mathbb{R}^{6} \times \mathbb{R}$, for some $\tilde{\beta}_{1}, \tilde{\beta}_{2}, \tilde{\beta}_{3}>0$.

Proof. By construction $\bar{V}(x, t)$ is homogeneous of degree 4 with respect to $\delta_{\lambda}^{\bar{r}}$, and $\partial \bar{V} / \partial t(x, t)+(\partial \bar{V} / \partial x) f\left(x, u_{n}(x, t)\right)$ is also homogeneous of degree 4 by Proposition 4. They are both continuous and periodic with period $T=2 \pi \epsilon$. The first inequality in the Proposition has already been obtained in (4.32). As shown, when $k_{x}, k_{y}$ satisfy (4.42), $\partial \bar{V} / \partial t(x, t)+(\partial \bar{V} / \partial x) f\left(x, u_{n}(x, t)\right)$ is negative definite. Corollary 1 then 
yields $\partial \bar{V} / \partial t(x, t)+(\partial \bar{V} / \partial x) f\left(x, u_{n}(x, t)\right) \leq-a \rho^{4}(x)$, where

$$
a=-\max _{\rho(x)=1,0 \leq t \leq T} \partial \bar{V} / \partial t(x, t)+(\partial \bar{V} / \partial x) f\left(x, u_{n}(x, t)\right) .
$$

The second inequality in the Proposition is then obtained with $\tilde{\beta}_{3}=a / \tilde{\beta}_{2}$ by application of the first inequality in the Proposition. This concludes the proof.

Having obtained a global strict Lyapunov function for the nominal system corresponding to (4.9), as in Proposition 15, we shall now proceed to the full system without uncertainties and disturbances, described by $f\left(x, u_{n}\right)$ and $g(x)$ in (4.9). We follow the approach in Du et al. [Du and S. Li, 2012] and Gui and Vukovich [Gui, Vukovich, and S. Xu, 2016]. Suppose that there exists a $\lambda_{0}>0$, such that

$$
L_{g} \bar{V}\left(\delta_{\lambda}^{r}(x), t\right) \leq \lambda^{4} \frac{\tilde{\beta}_{3}}{2} \Sigma,
$$

for all $(x, t) \in \mathbb{S}^{5} \times \mathbb{R}$, when $0<\lambda<\lambda_{0}$, where

$$
\Sigma=\min _{x \in \mathbb{S}^{5}, 0 \leq t \leq T} \bar{V}(x, t) .
$$

Define the set

$$
U_{0}=\left\{x \in \mathbb{R}: x=\delta_{\lambda}^{r}(\bar{x}), \bar{x} \in \mathbb{S}^{5}, 0 \leq \lambda<\lambda_{0}\right\} .
$$

Proposition 16 (Gui et al. (Gui, Vukovich, and S. Xu, 2016)). The set $U_{0}$ in (4.45) is open and contains the origin.

If (4.43) holds, then by the definition of $\Sigma$, we obtain

$$
L_{g} \bar{V}\left(\delta_{\lambda}^{r}(x), t\right) \leq \lambda^{4} \frac{\tilde{\beta}_{3}}{2} \bar{V}(x, t),
$$

for all $(x, t) \in \mathbb{S}^{5} \times \mathbb{R}$ when $0<\lambda<\lambda_{0}$. Any $x \neq 0$ can be written as $\delta_{\lambda}^{r}(\bar{x})$, for some $\lambda>0$ and $\bar{x} \in \mathbb{S}^{5}$. Hence, for arbitrary $x \neq 0,(4.46)$ leads to

$$
\begin{aligned}
L_{g} \bar{V}(x, t) & =L_{f} \bar{V}_{\alpha}\left(\delta_{\lambda}^{r}(\bar{x}), t\right) \leq \lambda^{4} \frac{\tilde{\beta}_{3}}{2} \bar{V}(\bar{x}, t), \\
& =\frac{\tilde{\beta}_{3}}{2} \bar{V}_{\alpha}\left(\delta_{\lambda}^{r}(\bar{x}), t\right),=\frac{\tilde{\beta}_{3}}{2} \bar{V}_{\alpha}(x, t),
\end{aligned}
$$


where homogeneity of $\bar{V}$ has been used. Continuity of $L_{f} \bar{V}$ and $\bar{V}$ imply that (4.47) holds for $x=0$ also with any $t \in \mathbb{R}$. As a result, combining Proposition 15 and $(4.47)$

$$
\frac{\partial \bar{V}}{\partial t}(x, t)+\frac{\partial \bar{V}}{\partial x}\left(f\left(x, u_{n}(x, t)\right)+g(x)\right) \leq-\frac{\tilde{\beta}_{3}}{2} \bar{V}(x, t)
$$

for all $(x, t) \in U_{0} \times \mathbb{R}$.

It remains to establish the condition in (4.43). Note that (4.43) is equivalent to the existence of $\lambda_{0}>0$, such that

$$
\frac{L_{g} \bar{V}\left(\delta_{\lambda}^{r}(x), t\right)}{\lambda^{4}} \leq \frac{\tilde{\beta}_{3}}{2} \Sigma
$$

for all $x \in \mathbb{S}^{5}$, when $0<\lambda<\lambda_{0}$. We have for $(x, t) \in \mathbb{S}^{5} \times[0, T]$ and $\lambda>0$

$$
\begin{aligned}
\frac{L_{g} \bar{V}\left(\delta_{\lambda}^{r}(x), t\right)}{\lambda^{4}} & =\frac{1}{\lambda^{4}} \sum_{i=1}^{6} \frac{\partial \bar{V}}{\partial x_{i}}\left(\delta_{\lambda}^{r}(x), t\right) g_{i}\left(\delta_{\lambda}^{r}(x)\right), \\
& =\sum_{i=1}^{6} \frac{\partial \bar{V}}{\partial x_{i}}(x, t) \frac{g_{i}\left(\delta_{\lambda}^{r}(x)\right)}{\lambda^{r_{i}}}
\end{aligned}
$$

where Proposition 3 has been used. Since $\partial \bar{V} / \partial x$ is continuous, it is uniformly bounded on $\mathbb{S}^{5} \times[0, T]$, which is compact. Hence, only the terms $g_{i}\left(\delta_{\lambda}^{r}(x)\right) / \lambda^{r_{i}}$ need to be considered for $i=1, \ldots, 6$. Related to the dilation $\delta_{\lambda}^{r}$, we define the reduced dilation for $q_{v}$ by $\delta_{\lambda}^{\tilde{r}}\left(q_{v}\right)=\left(\lambda q_{1}, \lambda q_{2}, \lambda^{2} q_{3}\right)$. From (4.9), we see that for $i=1,2$, $\left|g_{i}\left(\delta_{\lambda}^{r}(x)\right) / \lambda^{r_{i}}\right|$ is upper-bounded by terms of the form

$$
\begin{gathered}
\frac{\left|\lambda q_{j} \lambda^{2} \omega_{z}\right|}{2 \lambda}=\lambda^{2}\left|q_{j} \omega_{z}\right| / 2 \leq \lambda^{2} / 2, j=1,2, \\
\frac{\left|\lambda^{2} q_{3} \lambda \omega_{j}\right|}{2 \lambda}=\lambda^{2}\left|q_{3} \omega_{j}\right| / 2 \leq \lambda^{2} / 2, j=x, y, \\
\frac{\left|\left(\sqrt{1-\delta_{\lambda}^{\tilde{r}}\left(q_{v}\right)^{T} \delta_{\lambda}^{\tilde{r}}\left(q_{v}\right)}-1\right) \lambda \omega_{j}\right|}{2 \lambda} \leq\left|\omega_{j}\right||| \delta_{\lambda}^{\tilde{r}}\left(q_{v}\right) \| / 2 \leq \lambda / 2, \quad j=x, y
\end{gathered}
$$

where we have used the fact that $\left|q_{j}\right|,\left|\omega_{k}\right|,\left\|q_{v}\right\| \leq 1, j=1,2,3, k=x, y, z$ for $x \in \mathbb{S}^{5}$, and the fact that $\left\|\delta_{\lambda}^{\tilde{r}}\left(q_{v}\right)\right\| \leq\left\|\delta_{\lambda}^{\tilde{r}}\left(q_{v}\right)\right\|_{1} \leq \max \left\{\lambda, \lambda^{2}\right\}$ for $\left\|q_{v}\right\| \leq 1$, together with Proposition 7. Note that Proposition 7 can only be applied if $\left\|\delta_{\lambda}^{\tilde{r}}\left(q_{v}\right)\right\| \leq 1$, which requires that $\lambda \leq 1$. As a consequence, $\max \left\{\lambda, \lambda^{2}\right\}=\lambda$, and we require $\lambda_{0} \leq 1$. 
In a similar manner, for $i=3$, we obtain

$$
\frac{\left|\left(\sqrt{1-\delta_{\lambda}^{\tilde{r}}\left(q_{v}\right)^{T} \delta_{\lambda}^{\tilde{r}}\left(q_{v}\right)}-1\right) \lambda^{2} \omega_{z}\right|}{2 \lambda^{2}} \leq \lambda / 2
$$

Finally, for $i=5,6$, we obtain

$$
\frac{\left|g_{i}\left(\delta_{\lambda}^{\tilde{r}}(x)\right)\right|}{\lambda} \leq\left|c_{j}\right| \lambda^{2}, j= \begin{cases}x, & i=5 \\ y, & i=6\end{cases}
$$

Putting everything together, we obtain

$$
\frac{L_{g} \bar{V}\left(\delta_{\lambda}^{r}(x), t\right)}{\lambda^{4}} \leq b_{1} \lambda^{2}+b_{2} \lambda
$$

where $b_{1}=a_{1}+a_{2}+a_{5}\left|c_{x}\right|+a_{6}\left|c_{y}\right|, b_{2}=\left(a_{1}+a_{2}+a_{3}\right) / 2$ and

$$
a_{i}=\max _{S^{5} \times[0, T]}\left|\frac{\partial \bar{V}}{\partial x_{i}}(x, t)\right|
$$

for $i=1, \ldots, 6$. From (4.50), we now find that (4.49) is satisfied for $0<\lambda<\lambda_{0}$, when

$$
\lambda_{0}=\min \left\{1,\left(\sqrt{b_{2}^{2}+2 b_{1} \tilde{\beta}_{3} \Sigma}-b_{2}\right) /\left(2 b_{1}\right)\right\} .
$$

As result, we get the following.

Lemma 4.3. Consider the control law defined in (4.13), (4.28) and (4.29), where $k_{1}, k_{2}, k_{3}$, $k_{4}, k_{5}$, and $\epsilon$ satisfy the conditions stated in Proposition 14, and $k_{x}, k_{y}$ satisfy (4.42). Then, the closed-loop system defined by $\dot{x}=f\left(x, u_{n}(x, t)\right)+g(x)$ is locally exponentially stable.

Proof. By the previous development, $\bar{V}(x, t)$ as given in Proposition 15 satisfies (4.48) for all $(x, t) \in U_{0} \times \mathbb{R}$. By Remark 1 , there exists a $\chi>0$, such that the closed homogeneous ball $\overline{\mathcal{B}}_{\chi}(0)$ is contained in $U_{0}$. Let $x(0) \in \mathcal{B}_{\left(\tilde{\beta}_{1} / \tilde{\beta}_{2}\right)^{1 / 4} \chi}(0) \subset \mathcal{B}_{\chi}(0)$. Then, by Proposition $15, \bar{V}(x(0), 0) \leq \tilde{\beta}_{2} \rho^{4}(x(0))<\tilde{\beta}_{1} \chi^{4}$. Let $x(t)$ be the trajectory of $\dot{x}=f\left(x, u_{n}(x, t)\right)+g(x)$ resulting from initial condition $x(0)$. From Proposition 15 , we note that $\bar{V}(x(t), t)<\tilde{\beta}_{1} \chi^{4}$ implies that $x(t) \in \mathcal{B}_{\chi}(0)$. By (4.48), we have $\dot{\bar{V}}(x(t), t) \leq 0$ while $x(t) \in U_{0}$. Consequently, we have $\bar{V}(x(t), t) \leq \bar{V}(x(0), 0)<$ $\tilde{\beta}_{1} \chi^{4}$ while $x(t) \in U_{0}$. Since $x(t)$ cannot leave $U_{0}$ before leaving $\mathcal{B}_{\chi}(0)$ (the boundary 
of $\mathcal{B}_{\chi}(0)$ is contained in $\left.U_{0}\right)$, we must therefore have $x(t) \in \mathcal{B}_{\chi}(0)$ for all $t \geq 0$. Finally, in a similar manner as in the proof of Corollary 4.2.1, we obtain $\rho(x(t)) \leq$ $\left(\tilde{\beta}_{2} / \tilde{\beta}_{1}\right)^{1 / 4} \rho(x(0)) e^{-\left(\tilde{\beta}_{3} /\left(4 \tilde{\beta}_{2}\right)\right) t}$. This concludes the proof.

Next, we handle the uncertainties and disturbances contained in the terms $h(x, t$, $\left.\tau_{d}, \Delta J, \tilde{\omega}\right), D$ in (4.9). First, we define $\psi \in \mathbb{R}^{3}$ such that

$$
\bar{\psi}=\left[\begin{array}{c}
0_{3 \times 1} \\
\psi
\end{array}\right]=h\left(x, t, \tau_{d}, \Delta J, \tilde{\omega}\right)+D .
$$

We temporarily assume that there exist $\psi_{i m}>0$, such that $\left|\psi_{i}(t)\right| \leq \psi_{i m}$ for $i=1,2,3$. This will be made rigorous shortly.

Again, any non-zero $x \in U_{0}$ can be written as $x=\delta_{\lambda}^{r}(\bar{x})$, for some $\bar{x} \in \mathbb{S}^{5}$. Therefore, for any $(x, t) \in U_{0} \times \mathbb{R}$ with $x \neq 0$,

$$
\begin{aligned}
\frac{\partial \bar{V}}{\partial x}(x, t) \bar{\psi} & =\frac{\partial \bar{V}}{\partial x}\left(\delta_{\lambda}^{r}(\bar{x}), t\right) \bar{\psi} \\
& =\frac{\partial \bar{V}}{\partial \omega_{z}}\left(\delta_{\lambda}^{r}(\bar{x}), t\right) \psi_{1}+\frac{\partial \bar{V}}{\partial \omega_{x}}\left(\delta_{\lambda}^{r}(\bar{x}), t\right) \psi_{2}+\frac{\partial \bar{V}}{\partial \omega_{y}}\left(\delta_{\lambda}^{r}(\bar{x}), t\right) \psi_{3}, \\
& =\lambda^{2} \frac{\partial \bar{V}}{\partial \omega_{z}}(\bar{x}, t) \psi_{1}+\lambda^{3} \frac{\partial \bar{V}}{\partial \omega_{x}}(\bar{x}, t) \psi_{2}+\lambda^{3} \frac{\partial \bar{V}}{\partial \omega_{y}}(\bar{x}, t) \psi_{3}, \\
& \leq \lambda^{2} a_{4} \psi_{1 m}+\lambda^{3}\left(a_{5} \psi_{2 m}+a_{6} \psi_{3 m}\right), \\
& \leq \lambda^{2} \frac{a_{4} \psi_{1 m}}{\Sigma^{1 / 2}} \bar{V}^{1 / 2}(\bar{x}, t)+\lambda^{3} \frac{\left(a_{5} \psi_{2 m}+a_{6} \psi_{3 m}\right)}{\Sigma^{3 / 4}} \bar{V}^{3 / 4}(\bar{x}, t), \\
& =\frac{a_{4} \psi_{1 m}}{\Sigma^{1 / 2}}\left(\lambda^{4} \bar{V}(\bar{x}, t)\right)^{1 / 2}+\frac{\left(a_{5} \psi_{2 m}+a_{6} \psi_{3 m}\right)}{\Sigma^{3 / 4}}\left(\lambda^{4} \bar{V}(\bar{x}, t)\right)^{3 / 4}, \\
& =\frac{a_{4} \psi_{1 m}}{\Sigma^{1 / 2}} \bar{V}^{1 / 2}(x, t)+\frac{\left(a_{5} \psi_{2 m}+a_{6} \psi_{3 m}\right)}{\Sigma^{3 / 4}} \bar{V}^{3 / 4}(x, t),
\end{aligned}
$$

where Proposition 3, the homogeneity and periodicity of $\bar{V}, \partial \bar{V} / \partial x$ and the definitions of $\Sigma$ and $a_{i}$ in (4.44) and (4.51) have been made use of. By Proposition 3, $\left(\partial \bar{V} / \partial \omega_{i}\right)(0, t)=0$ for $i=x, y, z$ and for all $t \in \mathbb{R}$. Hence, (4.54) holds for $x=0$ also.

Consequently, combining (4.54) with (4.48), we find that

$$
\frac{\partial \bar{V}}{\partial t}(x, t)+\frac{\partial \bar{V}}{\partial x}\left(f\left(x, u_{n}(x, t)\right)+g(x)+\bar{\psi}\right) \leq-\bar{V}^{1 / 2}(x, t)\left(\frac{\tilde{\beta}_{3}}{2} \bar{V}^{1 / 2}(x, t)-c_{1} \bar{V}^{1 / 4}(x, t)-c_{2}\right),
$$


for all $(x, t) \in U_{0} \times \mathbb{R}$, where

$$
c_{1}=\frac{\left(a_{5} \psi_{2 m}+a_{6} \psi_{3 m}\right)}{\Sigma^{3 / 4}}, c_{2}=\frac{a_{4} \psi_{1 m}}{\Sigma^{1 / 2}}
$$

It is now straightforward to determine that the left-hand side of (4.55) is strictly negative when

$$
\bar{V}(x, t)>\mathrm{Y},
$$

where

$$
Y=\left(\frac{c_{1}+\sqrt{c_{1}^{2}+2 c_{2} \tilde{\beta}_{3}}}{\tilde{\beta}_{3}}\right)^{4} .
$$

With reference to the proof of Lemma 4.3, we require

$$
\mathrm{Y}<\tilde{\beta}_{1} \chi^{4}
$$

since if this holds, then in a manner similar to the proof of Lemma 4.3, (4.55) shows that for any initial $x(0) \in \mathcal{B}_{\left(\tilde{\beta}_{1} / \tilde{\beta}_{2}\right)^{1 / 4} \chi}(0)$, the resulting solution $x(t)$ of (4.9) cannot leave $\mathcal{B}_{\chi}(0)$. Furthermore, along such a trajectory,

$$
\limsup _{t \rightarrow \infty} \bar{V}(x(t), t) \leq \mathrm{Y}
$$

and by Proposition 15 it follows that

$$
\limsup _{t \rightarrow \infty} \rho(x(t)) \leq\left(\frac{Y}{\tilde{\beta}_{1}}\right)^{1 / 4}
$$

The final item required is to obtain suitable bounds $\psi_{i m}$. First, since we assume $q_{v}$ is available, we define the estimate of $x$

$$
\hat{x}=\left[q_{v} \hat{\omega}_{z} \hat{\omega}_{x} \hat{\omega}_{y}\right]^{T}
$$

The velocity-free control law is then defined as

$$
u(\hat{x}, t)=u_{n}(\hat{x}, t)=u_{n}(x, t)+\Delta u(x, t, \tilde{\omega}),
$$


where

$$
\Delta u(x, t, \tilde{\omega})=u_{n}(\hat{x}, t)-u_{n}(x, t),
$$

is a continuous periodic function of $(x, t, \tilde{\omega})$, satisfying $\Delta u(x, t, 0)=0$ for all $(x, t) \in$ $\mathbb{R}^{6} \times \mathbb{R}$.

Proposition 17. Fix $\bar{\chi}>0$ and define the set $W=\overline{\mathcal{B}}_{\bar{\chi}}(0) \times \mathbb{R} \times \bar{B}_{\tau_{d m}}(0)$. Then, for any $\zeta>0$ there exist $\varrho_{1}\left(\bar{\chi}, \tau_{d m}, \zeta\right), \varrho_{2}\left(\bar{\chi}, \tau_{d m}, \zeta\right)>0$ such that $\|\Delta J\| \leq \varrho_{1}\left(\bar{\chi}, \tau_{d m}, \zeta\right)$ together with $\|\tilde{\omega}\|<\varrho_{2}\left(\bar{\chi}, \tau_{d m}, \zeta\right)$ imply that $\left\|h\left(x, t, \tau_{d}, \Delta J, \tilde{\omega}\right)\right\|<\zeta$ for all $\left(x, t, \tau_{d}\right) \in W$.

Proof. Define the set $\bar{W}=\overline{\mathcal{B}}_{\bar{\chi}}(0) \times[0, T] \times \bar{B}_{\tau_{d m}}(0)$. First, note that the set $\bar{W}$ is compact in $\mathbb{R}^{6} \times \mathbb{R} \times \mathbb{R}^{3}$. Note that $h\left(x, t, \tau_{d}, 0,0\right)=0$ for all $\left(x, t, \tau_{d m}\right) \in \bar{W}$. Choose arbitrary $\zeta>0$. Continuity of $h$ on $\mathbb{R}^{3} \times \mathbb{R} \times \mathbb{R}^{3} \times \mathbb{R}^{3 \times 3} \times \mathbb{R}^{3}$ implies that for each point $z=\left(x, t, \tau_{d}\right) \in \bar{W}$, there exists $\varrho_{1, z}, \varrho_{2, z}>0$ and a neighborhood $V_{z} \subset \mathbb{R}^{3} \times \mathbb{R} \times \mathbb{R}^{3}$ of $z$, such that $\left\|h\left(\bar{x}, \bar{t}, \bar{\tau}_{d}, \Delta J, \tilde{\omega}\right)\right\|<\zeta$ for all $\left(\left(\bar{x}, \bar{t}, \bar{\tau}_{d}\right), \Delta J, \tilde{\omega}\right) \in V_{z} \times B_{\varrho_{1, z}}(0) \times B_{Q_{2, z}}(0)$. Next, the collection of all such sets $V_{z}$ (with $z \in \bar{W}$ ) form an open cover of $\bar{W}$. Since $\bar{W}$ is compact, there exist $z_{1}, \ldots, z_{n} \in W$, such that $\bar{W} \subset \bigcup_{i=1}^{n} V_{z i}$. By construction, $\left\|h\left(\bar{x}, \bar{t}, \bar{\tau}_{d}, \Delta J, \tilde{\omega}\right)\right\|<\zeta$ for all $\left(\left(\bar{x}, \bar{t}, \bar{\tau}_{d}\right), \Delta J, \tilde{\omega}\right) \in \bigcup_{i=1}^{n} V_{z i} \times B_{Q_{1, z i}}(0) \times B_{Q_{2, z i}}(0)$. Define $\varrho_{i}\left(\bar{\chi}, \tau_{d m}, \zeta\right)=\min \left\{\varrho_{i, z 1}, \ldots, \varrho_{i, z n}\right\}$ for $i=1,2$. Then, $\bar{W} \times B_{\varrho_{1}}(0) \times B_{\varrho_{2}}(0) \subset$ $\bigcup_{i=1}^{n} V_{z i} \times B_{\varrho_{1, z i}}(0) \times B_{Q_{2, z i}}(0)$, from which the conclusion follows by recognizing that $h$ is periodic and therefore the interval $[0, T]$ in the definition of $\bar{W}$ may be replaced by $\mathbb{R}$ to obtain the result on the set $W$. This concludes the proof.

Finally, we obtain the following result.

Lemma 4.4. Consider the control law defined in (4.13), (4.28) and (4.29), where $k_{1}, k_{2}, k_{3}$, $k_{4}, k_{5}, k_{x}, k_{y}, \epsilon$ satisfy the conditions stated in Proposition 15. Let $\chi>0$ be as defined in the proof of Lemma 4.3. Consider $\psi_{i m}>0$ for $i=1,2,3$, such that $Y$ as defined in (4.57) satisfies (4.58). Suppose that $\tau_{d m}$ satisfies $\tau_{d m} / J_{z}<\psi_{i 1}, \tau_{d m} / J_{x}<\psi_{i 2}$ and $\tau_{d m} / J_{y}<\psi_{i 3}$. Choose $\zeta>0$ such that $\zeta+\tau_{d m} / J_{z} \leq \psi_{i 1}, \zeta+\tau_{d m} / J_{x} \leq \psi_{i 2}$ and $\zeta+\tau_{d m} / J_{z} \leq \psi_{i 3}$. Choosing $\bar{\chi}>\chi$, obtain $\varrho_{1}\left(\bar{\chi}, \tau_{d m}, \zeta\right), \varrho_{2}\left(\bar{\chi}, \tau_{d m}, \zeta\right)>0$ from Proposition 17. Suppose that $\|\Delta J\|<\varrho_{1}\left(\bar{\chi}, \tau_{d m}, \zeta\right)$, and that $\|\tilde{\omega}(t)\|<\varrho_{2}\left(\bar{\chi}, \tau_{d m}, \zeta\right)$, for all $t \in[0, \bar{t}]$, for some $\bar{t} \in \mathbb{R}_{+} \bigcup\{\infty\}$. Let $x(0) \in \mathcal{B}_{\left(\tilde{\beta}_{1} / \tilde{\beta}_{2}\right)^{1 / 4} \chi}(0)$, with $x(t)$ the resulting trajectory of (4.9). 
Then, $x(t) \in \mathcal{B}_{\chi}(0)$ for $t \in[0, \bar{t}]$. Furthermore, if this holds for any $\bar{t} \geq 0$, then

$$
\limsup _{t \rightarrow \infty} \rho(x(t)) \leq\left(\frac{Y}{\tilde{\beta}_{1}}\right)^{1 / 4}
$$

Proof. By Proposition 17, and the stated conditions on $\zeta$ and $\tau_{d m}, \bar{V}(x, t)$ as given in Proposition 15 satisfies $(4.55)$ for all $(x, t) \in\left(\overline{\mathcal{B}}_{\bar{\chi}}(0) \cap U_{0}\right) \times \mathbb{R}$. Since $\left(\overline{\mathcal{B}}_{\bar{\chi}}(0) \cap U_{0}\right) \supset$ $\overline{\mathcal{B}}_{\chi}(0)$, it follows by similar arguments to those given following equation (4.58) that $x(t)$ cannot leave $\mathcal{B}_{\chi}(0)$ during the interval $[0, T]$. Finally, if this holds for any $\bar{t} \geq 0$, then the arguments leading up to (4.60) are valid. This concludes the proof.

\subsubsection{Observer design}

Let $\hat{q}, \hat{\omega}$ be the estimates of $q, \omega$ respectively, and define the attitude estimation error $\tilde{q}=\left(\tilde{q}_{v}, \tilde{q}_{4}\right)$ by $C(\tilde{q})=C(q) C^{T}(\hat{q})$ and $\tilde{\omega}=\omega-\hat{\omega}$. Let $\hat{q}$ satisfy

$$
\dot{\hat{q}}=\frac{1}{2} B(\hat{q}) p
$$

for some $p$, or equivalently [Hughes, 1986], $\dot{C}(\hat{q})=-p^{\times} C(\hat{q})$. Consequently, it is straightforward to show that $\dot{C}(\tilde{q})=-(\omega-C(\tilde{q}) p)^{\times} C(\tilde{q})$, which is equivalent to

$$
\dot{\tilde{q}}=B(\tilde{q})(\omega-C(\tilde{q}) p)
$$

The observer is now defined as

$$
\begin{aligned}
\dot{\hat{q}} & =\frac{1}{2} B(\hat{q}) C^{T}(\tilde{q})\left(\hat{\omega}+\gamma_{1} P^{-1}(\tilde{q}) \operatorname{sig}^{\alpha}\left(\tilde{q}_{v}\right)\right), \\
J_{n} \dot{\hat{\omega}} & =-\hat{\omega}^{\times} J_{n} \hat{\omega}+\tau_{g n}+\tau_{u}+\gamma_{2} J \operatorname{sig}^{\alpha_{1}}\left(\tilde{q}_{v}\right),
\end{aligned}
$$

where $\gamma_{1}, \gamma_{2}>0, \alpha \in(1 / 2,1), \alpha_{1}=2 \alpha-1$ and

$$
\tau_{g n}=3 \frac{\mu}{\|R\|^{5}}(C(q) R)^{\times} J_{n} C(q) R,
$$

is the nominal gravity-gradient torque. Note that $P^{-1}(\tilde{q})$ is invertible provided $\left\|\tilde{q}_{v}\right\|<1$. Since a local stability result is obtained, this restriction poses no problem. Utilizing (4.2), (4.5), (4.62), (4.63), (4.64), (4.65), and (4.66), one obtains the error 
equations

$$
\begin{aligned}
\dot{\tilde{q}}_{v} & =\frac{1}{2} B(\tilde{q})\left(\tilde{\omega}-\gamma_{1} P^{-1}(\tilde{q}) \operatorname{sig}^{\alpha}\left(\tilde{q}_{v}\right)\right), \\
\dot{\tilde{\omega}} & =-\gamma_{2} \operatorname{sig}^{\alpha_{1}}\left(\tilde{q}_{v}\right)-J_{n}^{-1}\left(\omega^{\times} J_{n} \omega-\hat{\omega}^{\times} J_{n} \hat{\omega}\right)+\tilde{\Omega}+J_{n}^{-1} \bar{\tau}_{d},
\end{aligned}
$$

where

$$
\begin{aligned}
\tilde{\Omega}= & J_{n}^{-1} \omega^{\times} J_{n} \omega-J^{-1} \omega^{\times} J \omega+\left(J^{-1}-J_{n}^{-1}\right) J_{n} H\left(u_{n}(x, t)+\Delta u(x, t, \tilde{\omega})\right) \\
& +J^{-1} \tau_{g}-J_{n}^{-1} \tau_{g n}+\left(J^{-1}-J_{n}^{-1}\right) \bar{\tau}_{d} .
\end{aligned}
$$

Next, using the definition of $\tilde{\omega}$, it is straightforward to show that

$$
\omega^{\times} J \omega-\hat{\omega}^{\times} J \hat{\omega}=\tilde{\omega}^{\times} J \omega+\omega^{\times} J \tilde{\omega}-\tilde{\omega}^{\times} J \tilde{\omega} .
$$

Finally, since a local stability result is pursued, with the assumption that $\left\|\tilde{q}_{v}\right\|<1$, choose $\tilde{q}_{4}>0$. Defining $\tilde{x}=\left[\tilde{q}_{v}^{T}, \tilde{\omega}^{T}\right]^{T}$, the observer error dynamics in (4.68) and (4.69) can be written as

$$
\dot{\tilde{x}}=g_{\alpha}(\tilde{x})+\tilde{f}\left(\tilde{q}_{v}, \tilde{\omega}, \omega\right)+\tilde{h}\left(x, t, \bar{\tau}_{d}, r, \tilde{\omega}, \Delta J\right)+\tilde{d},
$$

where

$$
\begin{gathered}
g_{\alpha}(\tilde{x})=\left[\begin{array}{c}
g_{1 \alpha}(\tilde{x}) \\
g_{2 \alpha}(\tilde{x})
\end{array}\right], \tilde{f}\left(\tilde{q}_{v}, \tilde{\omega}, \omega\right)=\left[\begin{array}{c}
\tilde{f}_{1}\left(\tilde{q}_{v}, \tilde{\omega}\right) \\
\tilde{f}_{2}(\tilde{\omega}, \omega)
\end{array}\right], \\
\tilde{h}\left(x, t, \bar{\tau}_{d}, R, \tilde{\omega}, \Delta J\right)=\left[\begin{array}{c}
0_{3 \times 1} \\
\tilde{\Omega}
\end{array}\right], \tilde{d}=\left[\begin{array}{c}
0 \\
J_{n}^{-1} \bar{\tau}_{d}
\end{array}\right], \\
g_{1 \alpha}(\tilde{x})=\tilde{\omega} / 2-\gamma_{1} \operatorname{sig}^{\alpha}\left(\tilde{q}_{v}\right), g_{2 \alpha}(\tilde{x})=-\gamma_{2} \operatorname{sig}^{\alpha_{1}}\left(\tilde{q}_{v}\right) \\
\tilde{f}_{1}\left(\tilde{q}_{v}, \tilde{\omega}\right)=\frac{1}{2}\left(A\left(\tilde{q}_{v}\right)-I\right) \tilde{\omega}, \\
\tilde{f}_{2}(\tilde{\omega}, \omega)=-J_{n}^{-1}\left(\tilde{\omega}^{\times} J_{n} \omega+\omega^{\times} J_{n} \tilde{\omega}-\tilde{\omega}^{\times} J_{n} \tilde{\omega}\right) .
\end{gathered}
$$

Note that

$$
\tilde{h}\left(x, t, \bar{\tau}_{d}, R, \tilde{\omega}, 0\right)=0,
$$


for all $\left(x, t, \bar{\tau}_{d}, R, \tilde{\omega}\right) \in \mathbb{R}^{6} \times \mathbb{R} \times \mathbb{R}^{3} \times\left(\mathbb{R}^{3} \backslash\{0\}\right) \times \mathbb{R}^{3}$. It can be verified that $g_{\alpha}$ is homogeneous of degree $k=\alpha-1$ with respect to the dilation $\delta_{\lambda}^{r_{e}(\alpha)}(\tilde{x})=\left[\lambda \tilde{q}_{v}^{T}, \lambda^{\alpha} \tilde{\omega}^{T}\right]^{T}$. For a spacecraft in a bounded orbit about a primary body, the following assumption can be made.

Assumption 4.5. There exist $0<R_{m}<R_{M}$ such that $R(t) \in O$ for all $t \geq 0$, where $O=\left\{R \in \mathbb{R}^{3}: R_{m} \leq\|R\| \leq R_{M}\right.$.

Proposition 18 (Chapter 3). Given $\gamma_{1}, \gamma_{2}>0$ and $\ell \geq 2$, there exists $\bar{\alpha} \in(1 / 2,1)$ with corresponding $V_{\alpha}: \mathbb{R}^{n} \rightarrow \mathbb{R}$ satisfying the following properties for $\alpha \in(\bar{\alpha}, 1)$ :

1. $V_{\alpha}$ is positive-definite and radially unbounded.

2. $V_{\alpha}$ is homogeneous of degree $\ell$ with respect to the dilation $\delta_{\lambda}^{r_{e}(\alpha)}$.

3. $V_{\alpha}$ is infinitely differentiable on $\mathbb{R}^{n} \backslash\{0\}$ and continuously differentiable at $\tilde{x}=0$.

4. $L_{g_{\alpha}} V_{\alpha}(\tilde{x}) \leq-c(\alpha) V_{\alpha}^{(\ell+k) / \ell}(\tilde{x})$ for all $\tilde{x} \in \mathbb{R}^{6}$, with $c(\alpha)>0$.

5. Given $\bar{\chi}>0$, there exists an open set $U(\bar{\chi})$ containing the origin such that $\|x\| \leq \bar{\chi}$ implies that

$$
L_{g_{\alpha}+\tilde{f}} V_{\alpha}(\tilde{x}) \leq-\frac{c(\alpha)}{2} V_{\alpha}^{(\ell+k) / \ell}(\tilde{x})
$$

for all $\tilde{x} \in U(\bar{\chi})$.

While for a given bound $\bar{\chi}$, it has been established that (4.72) holds on the open set $U(\bar{\chi})$, it will be useful to find an open sublevel set of $V_{\alpha}$, contained in $U(\bar{\chi})$, since this will then become an invariant set, and in the absence of uncertainty and disturbance, will be inside the domain of attraction of the observer. To this end, since $U(\bar{\chi})$ is open, let $\eta>0$ be the radius of a closed ball contained inside $U(\bar{\omega})$. Let $\bar{\kappa}=\min _{\tilde{x} \in \eta S^{5}} V_{\alpha}(\tilde{x})>0$. The set

$$
W=\left\{\tilde{x} \in B_{\eta}(0): V_{\alpha}(\tilde{x})<\bar{\kappa}\right\}
$$

is such a set.

Finally the terms $\tilde{h}$ and $\tilde{d}$ in (4.70) are handled. Similar to Proposition 17, we have the following result. 
Proposition 19. Fix $\bar{\chi}, \bar{\eta}>0$ and define the set $Y=\overline{\mathcal{B}}_{\bar{\chi}}(0) \times[0, T] \times B_{\bar{\tau}_{d m}}(0) \times$ $O \times \bar{B}_{\bar{\eta}}(0)$. Then, for any $\xi>0$ there exist $\varrho_{3}\left(\bar{\chi}, \bar{\tau}_{d m}, \bar{\eta}, \xi\right)>0$ such that $\|\Delta J\| \leq$ $\varrho_{3}\left(\bar{\chi}, \bar{\tau}_{d m}, \bar{\eta}, \bar{\xi}\right)$ implies that $\|\tilde{\Omega}\|=\left\|\tilde{h}\left(x, t, \bar{\tau}_{d}, R, \tilde{\omega}, \Delta J\right)\right\|<\xi$ for all $\left(x, t, \bar{\tau}_{d}, R, \tilde{\omega}\right) \in Y$.

Using Proposition 3, for any $\tilde{x} \in \mathbb{S}^{5}$ and $\lambda>0$ small enough that $\delta_{\lambda}^{r_{e}(\alpha)}(\tilde{x}) \in$ $\bar{B}_{\eta}(0)$, one has from (4.71) and Proposition 18 that when $\bar{\eta} \geq \eta$ in the definition of the set $W$ in (4.73),

$$
\begin{aligned}
L_{\tilde{h}+\tilde{d}} V_{\alpha}\left(\delta_{\lambda}^{r_{e}(\alpha)}(\tilde{x})\right) & =\frac{\partial V_{\alpha}}{\partial \tilde{\omega}}\left(\delta_{\lambda}^{r_{e}(\alpha)}(\tilde{x})\right)\left(\tilde{\Omega}+J_{n}^{-1} \bar{\tau}_{d}\right), \\
& =\lambda^{\ell-\alpha} \frac{\partial V_{\alpha}}{\partial \tilde{\omega}}(\tilde{x})\left(\tilde{\Omega}+J_{n}^{-1} \bar{\tau}_{d}\right), \\
& \leq \lambda^{\ell-\alpha}\left\|\frac{\partial V_{\alpha}}{\partial \tilde{\omega}}(\tilde{x})\right\|\left(\xi+\left\|J_{n}^{-1}\right\| \bar{\tau}_{d}\right),
\end{aligned}
$$

when $(x, t) \in \overline{\mathcal{B}}_{\bar{\chi}}(0) \times \mathbb{R}$, provided $\|\Delta J\|<\varrho_{3}\left(\bar{\chi}, \bar{\tau}_{d m}, \bar{\eta}, \bar{\xi}\right)$. Define

$$
a=\max _{\tilde{x} \in S^{5}}\left\|\frac{\partial V_{\alpha}}{\partial \tilde{\omega}}(\tilde{x})\right\|, \quad b=\min _{\tilde{x} \in \mathbb{S}^{5}} V_{\alpha}^{(\ell-\alpha) / \ell}(\tilde{x})>0
$$

By definition of $b, V_{\alpha}^{(\ell-\alpha) / \ell}(\tilde{x}) / b \geq 1$ for all $\tilde{x} \in \mathbb{S}^{5}$. Using these bounds in (4.74) leads to

$$
L_{\tilde{d}} V_{\alpha}\left(\delta_{\lambda}^{r(\alpha)}(\tilde{x})\right) \leq \frac{a\left(\tilde{\xi}+\left\|J_{n}^{-1}\right\| \bar{\tau}_{d m}\right)}{b} \lambda^{\ell-\alpha} V_{\alpha}^{(\ell-\alpha) / \ell}(\tilde{x}),
$$

for all $\tilde{x} \in \mathbb{S}^{5}$ with $\lambda>0$ small enough that $\delta_{\lambda}^{r_{e}(\alpha)}(\tilde{x}) \in \bar{B}_{\eta}(0)$, provided $(x, t) \in$ $\overline{\mathcal{B}}_{\bar{\chi}}(0) \times \mathbb{R}$ and $\|\Delta J\|<\varrho_{3}\left(\bar{\chi}, \bar{\tau}_{d m}, \bar{\eta}, \bar{\xi}\right)$. Using the same arguments as surrounding equation (4.54), one finds that

$$
L_{\tilde{h}+\tilde{d}} V_{\alpha}(\tilde{x}) \leq \frac{a\left(\tilde{\xi}+\left\|J_{n}^{-1}\right\| \bar{\tau}_{d m}\right)}{b} V_{\alpha}^{(\ell-\alpha) / \ell}(\tilde{x})
$$

for all $\tilde{x} \in \bar{B}_{\eta}(0)$, provided $(x, t) \in \overline{\mathcal{B}}_{\bar{\chi}}(0) \times \mathbb{R}$ and $\|\Delta J\|<\varrho_{3}\left(\bar{\chi}, \bar{\tau}_{d m}, \bar{\eta}, \xi\right)$.

Finally, combining (4.72) and (4.75), one finds that when $(x, t) \in \overline{\mathcal{B}}_{\bar{\chi}}(0) \times \mathbb{R}$ and $\|\Delta J\|<\varrho_{3}\left(\bar{\chi}, \bar{\tau}_{d m}, \bar{\eta}, \xi\right)$, for trajectories $\tilde{x}(t)$ inside $B_{\eta}(0)$ we have

$$
\begin{aligned}
\dot{V}_{\alpha}(\tilde{x}(t)) & =L_{g_{\alpha}+\tilde{f}} V_{\alpha}(\tilde{x})+L_{\tilde{h}+\tilde{d}} V_{\alpha}(\tilde{x}(t)) \\
& \leq-\frac{c(\alpha)}{2} V_{\alpha}^{(\ell+\alpha-1) / \ell}(\tilde{x})+\frac{a\left(\xi+\left\|J_{n}^{-1}\right\| \bar{\tau}_{d m}\right)}{b} V_{\alpha}^{(\ell-\alpha) / \ell}(\tilde{x}) \\
& =-V_{\alpha}^{(\ell-\alpha) / \ell}(\tilde{x})\left(\frac{c(\alpha)}{2} V_{\alpha}^{(2 \alpha-1) / \ell}(\tilde{x})-\frac{a\left(\xi+\left\|J_{n}^{-1}\right\| \bar{\tau}_{d m}\right)}{b}\right) .
\end{aligned}
$$


From this is is seen that $\dot{V}_{\alpha}<0$ when

$$
V_{\alpha}(\tilde{x})>\left(\frac{2 a\left(\xi+\left\|J_{n}^{-1}\right\| \bar{\tau}_{d m}\right)}{c(\alpha) b}\right)^{\ell /(2 \alpha-1)} .
$$

Consequently, to guarantee feasibility for (4.77), the following restriction is made

$$
\left(\frac{2 a\left(\xi+\left\|J_{n}^{-1}\right\| \bar{\tau}_{d m}\right)}{c(\alpha) b}\right)^{\ell /(2 \alpha-1)}<\bar{\kappa}
$$

This is clearly satisfied if $\bar{\tau}_{d m}>0$ and $\xi>0$ are small enough. The following result is obtained.

Lemma 4.6. Choose $\alpha \in(\bar{\alpha}, 1)$. Suppose that $x(t) \in \overline{\mathcal{B}}_{\bar{\chi}}(0)$ for all $t \in[0, \bar{t}]$, for some $\bar{t} \in \mathbb{R}_{+} \bigcup\{\infty\}$. Select $\eta>0$ such that $\bar{B}_{\eta}(0) \subset U(\bar{\chi})$ and obtain $\bar{\kappa}=\min _{\tilde{x} \in \eta S^{5}} V_{\alpha}(\tilde{x})$. Selecting $\bar{\eta}>\eta$, suppose that $\bar{\tau}_{d m}>0$ is small enough that $\xi>0$ can be selected such that (4.78) is satisfied, and obtain corresponding $\varrho_{3}\left(\bar{\chi}, \bar{\tau}_{d m}, \bar{\eta}, \xi\right)$ from Proposition 18. Finally, suppose $\|\Delta J\|<\varrho_{3}\left(\bar{\chi}, \bar{\tau}_{d m}, \bar{\eta}, \xi\right)$. If $\tilde{x}(0) \in W$ where $W$ is defined in (4.73), then $V_{\alpha}(\tilde{x}(t))<\bar{\kappa}$ and $\|\tilde{x}(t)\|<\eta$ for all $t \in[0, \bar{t}]$. If this holds for any $\bar{t} \geq 0$, then

$$
\limsup _{t \rightarrow \infty} V_{\alpha}(\tilde{x}(t)) \leq\left(\frac{2 a\left(\xi+\left\|J_{n}^{-1}\right\| \bar{\tau}_{d m}\right)}{c(\alpha) b}\right)^{\ell /(2 \alpha-1)}, \text { and } \limsup _{t \rightarrow \infty}\|\tilde{x}(t)\| \leq \eta_{\ell}<\eta
$$

where

$$
\eta_{\ell}=\max _{\tilde{x} \in F}\|\tilde{x}\|, \quad F=\left\{\tilde{x} \in B_{\eta}(0): V_{\alpha}(\tilde{x}) \leq\left(\frac{2 a\left(\tilde{\zeta}+\left\|J_{n}^{-1}\right\| \bar{\tau}_{d m}\right)}{c(\alpha) b}\right)^{\ell /(2 \alpha-1)}\right\} .
$$

Proof. This can be obtained using similar arguments as in the proof of Lemma 4.4, noting that $\tilde{x}(t) \in W \subset B_{\eta}(0)$ for all $t \in[0, \bar{t}]$ (see the definition of $W$ in (4.73)), and the fact that if this holds for any $\bar{t} \geq 0, \tilde{x}(t) \rightarrow F \subset \bar{B}_{\eta_{\ell}}(0)$ as $t \rightarrow \infty$. This concludes the proof.

\subsection{Main Result}

The main result can now be presented.

Theorem 4.7. Consider the system described by (4.1) and (4.2), together with the observerbased control law in (4.5), (4.13), (4.28), (4.29), (4.62), (4.65) and (4.66). Then, provided 
CHAPTER 4. Inertial Attitude Stabilization using a Generalized Time-Varying

Nonlinear Controller

the disturbance torque $\tau_{d}$ and inertia uncertainty $\Delta J$ are small enough, the control and observer parameters can be chosen such that the closed-loop system states are locally ultimately bounded.

Proof. Choose control law parameters $k_{1}, k_{2}, k_{3}, k_{4}, k_{5}, k_{x}, k_{u}, \epsilon$ as in the statement of Lemma 4.4. In addition, for small enough $\tau_{d m}>0$, it is possible to choose parameters $\chi, \bar{\chi}, \zeta$, as in the statement of Lemma 4.4 , and obtain related $\varrho_{1}\left(\bar{\chi}, \tau_{d m}, \zeta\right), \varrho_{2}\left(\bar{\chi}, \tau_{d m}, \zeta\right)$ also as in Lemma 4.4 .

For the observer, choose $\alpha \in(\bar{\alpha}, 1)$, and $\gamma_{1}, \gamma_{2}>0$. Choose $\eta>0$ as in Lemma 3, with the added restriction that $\eta \leq \varrho_{2}\left(\bar{\chi}, \tau_{d m}, \zeta\right)$ and obtain $\bar{\kappa}>0$ as in Lemma 3. Selecting $\bar{\eta}>\eta$, suppose that $\bar{\tau}_{d m}>0$ is small enough that $\xi>0$ can be selected such that (4.78) is satisfied, and obtain corresponding $\varrho_{3}\left(\bar{\chi}, \bar{\tau}_{d m}, \bar{\eta}, \bar{\xi}\right)$ from Proposition 18. Finally, suppose $\|\Delta J\|<\min \left\{\varrho_{1}\left(\bar{\chi}, \tau_{d m}, \zeta\right), \varrho_{3}\left(\bar{\chi}, \bar{\tau}_{d m}, \bar{\eta}, \xi\right)\right\}$.

Now, consider the set $H=\mathcal{B}_{\chi}(0) \times B_{\eta}(0)$, and consider the initial conditions $x(0) \in$ $\mathcal{B}_{\left(\tilde{\beta}_{1} / \tilde{\beta}_{2}\right)^{1 / 4} \chi}(0)$ (as in Lemma 4.4), and $\tilde{x}(0) \in W$ (as in Lemma 3). It is clear that $(x(0), \tilde{x}(0)) \in H$, and that therefore $(x(t), \tilde{x}(t))$ must remain in $H$ over some interval $[0, \bar{t})$, with $\bar{t}>0$. By Lemmas 4.4 and 4.6 , it can be inferred that $x(t)$ cannot reach the boundary of $\mathcal{B}_{\chi}(0)$ before $\tilde{x}(t)$ reaches the boundary of $B_{\eta}(0)$, and vice-versa. So, the only possibility for $(x(t), \tilde{x}(t))$ to leave $H$ is if $x(t)$ and $\tilde{x}(t)$ both reach the respective boundaries of $\mathcal{B}_{\chi}(0)$ and $B_{\eta}(0)$ at the same time instant. Let $\bar{t}>0$ be that time instant. We then have $\rho(x(\bar{t}))=\chi$ and $\|\tilde{x}(\bar{t})\|=\eta$, while $(x(t), \tilde{x}(t)) \in H$ for $0 \leq t<\bar{t}$. By definition of $\bar{\chi}$, we therefore have $x(t) \in \overline{\mathcal{B}}_{\bar{\chi}}(0)$ for $t \in[0, \bar{t}]$. By Lemma 3, it then follows that $\|\tilde{x}(\bar{t})\|<\eta$, and subsequently by Lemma 4.4 that $x(\bar{t}) \in \mathcal{B}_{\chi}(0)$, which is a contradiction. Hence, we see that $(x(t), \tilde{x}(t)) \in H$ for all $t \geq 0$. By Lemmas 4.4 and 4.6 , it then follows that they are ultimately bounded. This concludes the proof.

\subsection{Numerical Examples}

This section presents numerical examples to demonstrate the performance of the proposed observer-based controller under realistic implementation conditions, including sampled measurements with measurement noise, sample and hold control implementation, uncertainties in the spacecraft inertia matrix, aerodynamic and 
CHAPTER 4. Inertial Attitude Stabilization using a Generalized Time-Varying

Nonlinear Controller

solar pressure disturbance torques and small orbital eccentricities. For comments on practical implementations, one can refer Section 3.5.

The spacecraft model is taken from Godard et al. [Godard, K. Kumar, and A. Zou, 2013]. The spacecraft is in a Keplerian orbit with semi-major axis $a=6878 \mathrm{~km}$, and initial condition at perigee. The spacecraft nominal moments of inertia used in the control and observer design are $J_{x}=0.0020 \mathrm{~kg} \cdot \mathrm{m}^{2}, J_{y}=0.0017 \mathrm{~kg} \cdot \mathrm{m}^{2}$, and $J_{z}=0.0015 \mathrm{~kg} \cdot \mathrm{m}^{2}$. The aerodynamic and solar pressure torques are given by

$$
\begin{gathered}
\tau_{a}=1.36 A_{f}\left[\begin{array}{c}
1+\sin \left(\omega_{0} t\right)+0.5 \sin \left(2 \omega_{0} t\right) \\
-\left(5 \times 10^{2}\right)\left[4+2 \sin \left(\omega_{0} t\right)+0.5 \sin \left(2 \omega_{0} t\right)\right] \\
-\left[1+2 \sin \left(\omega_{0} t\right)+0.5 \sin \left(2 \omega_{0} t\right)\right]
\end{array}\right], \\
\tau_{s}=S_{f}\left[\begin{array}{c}
\left(2 \times 10^{-5}\right)\left[1-2 \sin \left(\omega_{0} t\right)\right] \\
\left(1 \times 10^{-3}\right) \cos \left(\omega_{0} t\right) \\
-\left(5 \times 10^{-5}\right) \cos \left(\omega_{0} t\right)
\end{array}\right],
\end{gathered}
$$

respectively, where $A_{f}=1.0 \times 10^{-11}$ and $S_{f}=1.70 \times 10^{-6}$ are derived from "worstcase scenarios" for the RyeSat spacecraft model as outlined in [Godard, K. Kumar, and A. Zou, 2013]. Table 4.1 shows the control and observer parameters.

\begin{tabular}{|c|c|}
\hline Parameter & Value \\
\hline $\begin{array}{c}a \\
\left(k_{1}, k_{2}, k_{3}, k_{4}, k_{5}, k_{x}, k_{y}\right) \\
\left(\alpha, \gamma_{1}, \gamma_{2}\right) \\
{\left[J_{n x}, J_{n y}, J_{n z}\right]}\end{array}$ & $\begin{array}{c}6878 \mathrm{~km} \\
(0.04,0.04,0.04,0.1,5,0.05,0.05) \\
(0.9,0.04,0.02) \\
{[0.0020,0.0017,0.0015] \mathrm{kg} \cdot \mathrm{m}^{2}}\end{array}$ \\
\hline $\begin{array}{c}\text { Nominal Disturbance-Free } \\
\epsilon(0), \omega_{b I}(0)\end{array}$ & {$[0.5245,0.5915,0.1585]^{T}, 0 \mathrm{rad} / \mathrm{s}$} \\
\hline $\begin{array}{c}\text { Nominal Disturbance } \\
\epsilon(0), \omega_{b I}(0) \\
{\left[\tau_{a}, \tau_{s}\right]}\end{array}$ & $\begin{array}{c}{[0.5245,0.5915,0.1585]^{T}, 0 \mathrm{rad} / \mathrm{s}} \\
\text { time-varying }\end{array}$ \\
\hline $\begin{array}{c}\text { Disturbances and Uncertainties } \\
\operatorname{diag}\{\Delta J\} \\
\Delta q_{v}, \epsilon(0), \omega_{b I}(0), \hat{\omega}_{b I}(0)\end{array}$ & $\begin{array}{c} \pm 5 \% \\
\pm 5 \mathrm{deg}, \pm 180 \mathrm{deg}, \pm 5 \mathrm{deg} s^{-1}, \pm 1.4 \mathrm{deg} s^{-1}\end{array}$ \\
\hline
\end{tabular}

TABLE 4.1: Summary of parameters 
In the numerical examples, attitude measurements are generated at sample times $t_{k}(k=0,1, \ldots)$ according to

$$
\begin{aligned}
& q_{v m}\left(t_{k}\right)=q_{4}\left(t_{k}\right) q_{v e, k}+q_{4 e, k} q_{v}\left(t_{k}\right)+q_{v}\left(t_{k}\right)^{\times} q_{v e, k} \\
& q_{4 m}\left(t_{k}\right)=q_{4}\left(t_{k}\right) q_{4 e, k}-q_{v}\left(t_{k}\right)^{T} q_{v e, k}
\end{aligned}
$$

where $q_{v e, k}$ is generated with MATLAB's "randn" random number generator, with standard deviation of $0.1 \times 180 /(6 \pi)$ in each component and $q_{4 e, k}=\sqrt{1-q_{v e, k}^{T} q_{v e, k}}$. Consequently, $\left(q_{v e, k}, q_{4 e, k}\right)$ is a unit quaternion representing a random rotational measurement error with 0.1 degree error (3-sigma).

The sampled-data implementation of the proposed observer-based control law is

$$
\begin{aligned}
\tau_{u}(t) & \left.=J_{n} H u_{n}\left(x_{m}\left(t_{k}\right), t_{k}\right)\right), \\
\dot{\hat{q}}(t) & =\frac{1}{2} B(\hat{q}(t)) C^{T}\left(\tilde{q}_{m}(t)\right)\left(\hat{\omega}(t)+\gamma_{1} P^{-1}\left(\tilde{q}_{m}(t)\right) \operatorname{sig}^{\alpha}\left(\tilde{q}_{v m}(t)\right)\right), \\
J \dot{\hat{\omega}}(t) & =-\hat{\omega}(t)^{\times} J \hat{\omega}(t)+\tau_{g, m}\left(t_{k}\right)+\tau_{u}(t)+\gamma_{2} J \operatorname{sig}^{\alpha_{1}}\left(\tilde{q}_{v m}(t)\right),
\end{aligned}
$$

then for $t \in\left[t_{k}, t_{k+1}\right)$, where

$$
x_{m}\left(t_{k}\right)=\left[\begin{array}{llll}
q_{v m}^{T}\left(t_{k}\right) & \hat{\omega}_{z}\left(t_{k}\right) & \hat{\omega}_{x}\left(t_{k}\right) & \hat{\omega}_{y}\left(t_{k}\right)
\end{array}\right]^{T} .
$$

and $\tilde{q}_{m}=\left(\tilde{q}_{v m}, \tilde{q}_{4 m}\right)$ is given by

$$
\begin{aligned}
& \tilde{q}_{v m}(t)=-q_{4 m}\left(t_{k}\right) \hat{q}_{v}(t)+\hat{q}_{4}(t) q_{v m}\left(t_{k}\right)-\hat{q}_{v}(t)^{\times} q_{v m}\left(t_{k}\right), \\
& \tilde{q}_{4 m}(t)=q_{4 m}\left(t_{k}\right) \hat{q}_{4}(t)+q_{v m}\left(t_{k}\right)^{T} \hat{q}_{v}(t) .
\end{aligned}
$$

The sample period in this chapter is chosen to be $\Delta T=t_{k+1}-t_{k}=0.1$ seconds, measurement limitations outlined in Section 3.5 also applies here. Numerical integration is performed using a 4th order Runge-Kutta method with step size equal to $\Delta T$. Smaller step sizes were also tested, but did not produce any noticeable change in results. 


\subsubsection{Nominal disturbance-free case}

The purpose of the example in this subsection is two-fold: (1) to demonstrate the performance of the observer-based control law under ideal conditions (perfect knowledge of the spacecraft inertia matrix, no external disturbances other than gravity-gradient); (2) to compare the performance of the ideal continuous-time (not sampled-data) observer-based control in the absence of measurement noise with the sampled-data observer-based control with noisy measurements. The spacecraft attitude relative to the orbiting frame is given by $q_{v}(0)=[0.5245,0.5915,0.1585]$, $q_{4}(0)=\sqrt{1-q_{v}(0)^{T} q_{v}(0)}$, while the initial orbital angular velocity is given by $\omega(0)=0 \mathrm{rad} / \mathrm{s}$.

Figure 4.1 shows the resulting attitude and angular velocity responses, respectively. It can be seen that the attitude and angular velocity responses are similar, with the sampled-data responses exhibiting some noise, which is due to the presence of noise on the measurements.The sampled-data approximates continuous-time control, attributing to the finite-time observer-based controller. Having established this, the examples presented in the remaining subsections are for the sampled-data controller only.

\subsubsection{Nominal case with disturbances}

The purpose the example in this subsection is to demonstrate the performance of the sampled-data observer-based control law under nominal conditions (perfect knowledge of the spacecraft inertia matrix, and gravity-gradient torque), but with the inclusion of the aerodynamic and solar pressure disturbance torques. The initial conditions are the same as in Section 4.4.1. Figure 4.2 shows the resulting attitude and angular velocity responses. Figure 3 shows the spacecraft control torques. Comparing Figure 4.2 to Figure 4.1, it can be seen that the while the attitude and angular velocity errors still converge to a neighborhood of the origin, the presence of the disturbance torques results in slightly larger steady-state attitude and angular velocity errors, which is to be expected since the disturbance torques act about all three spacecraft axes, while the control torque is limited to the roll and pitch axes. 

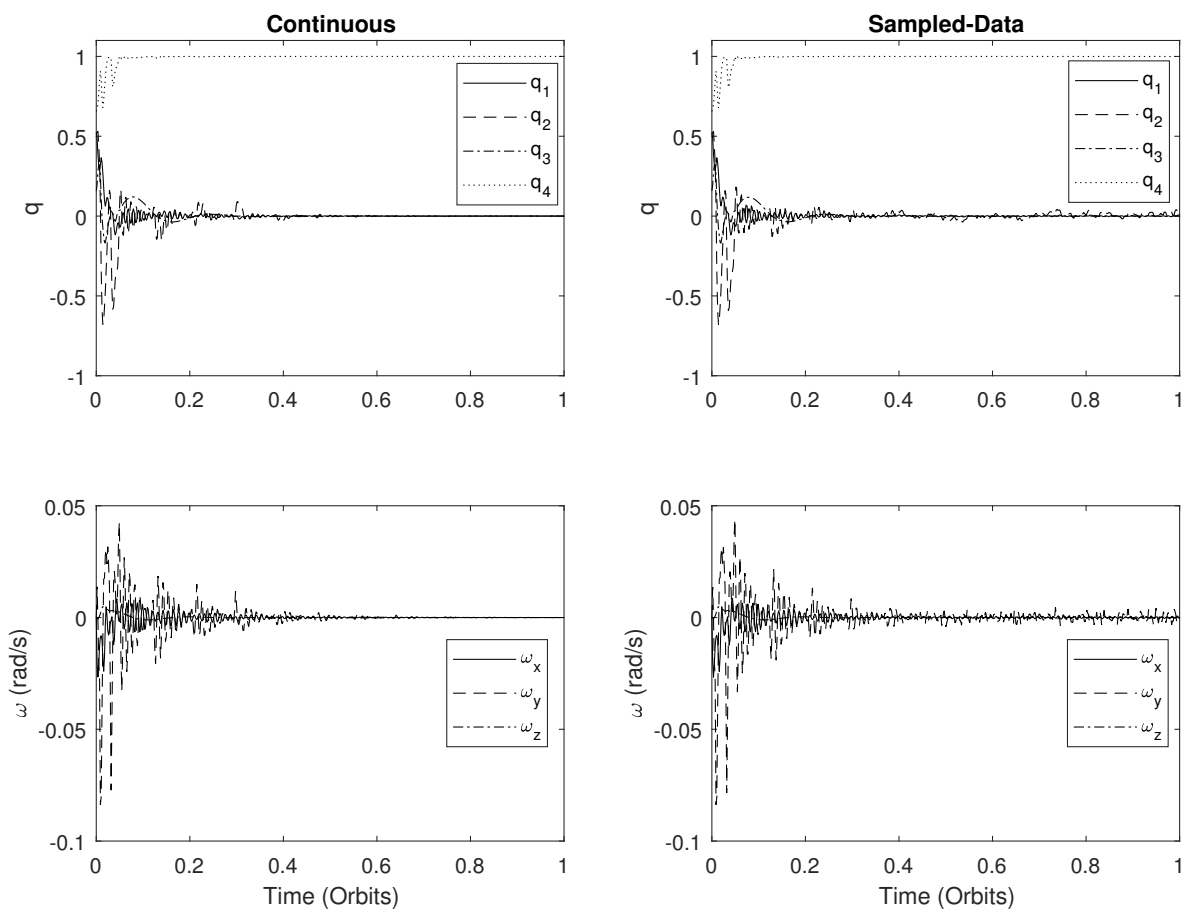

FIGURE 4.1: Attitude and angular velocity responses in nominal disturbance-free case
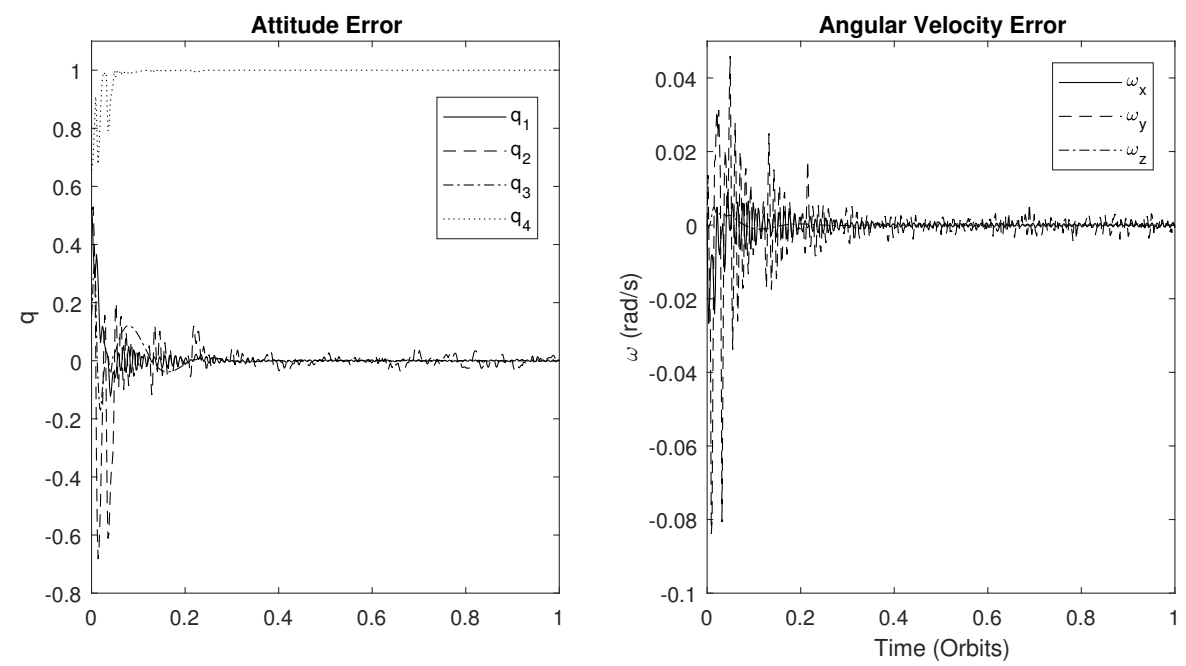

FIGURE 4.2: Attitude and angular velocity responses in nominal case with disturbances

\subsubsection{All uncertainties and disturbances}

The purpose of the example in this subsection is to demonstrate the robustness of the sampled-data observer-based control law to uncertainties in the spacecraft inertia matrix, in the presence of measurement noise for a variety of initial conditions with large initial attitude errors. To this end, one hundred simulations are performed, 


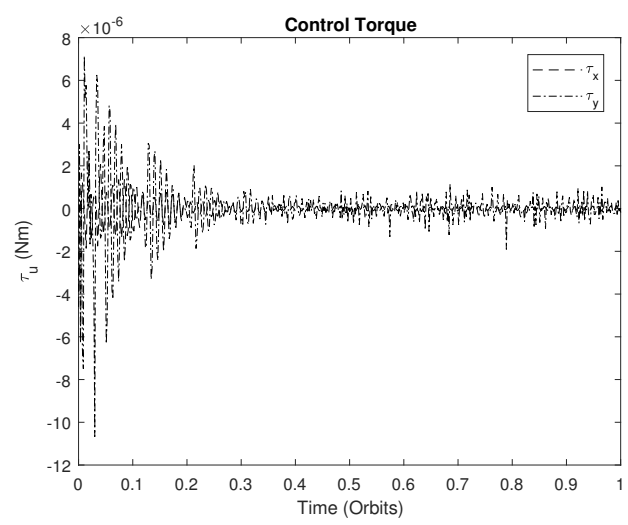

FIGURE 4.3: Control torque in nominal case with disturbances

where the true spacecraft inertia matrix, initial conditions are randomly generated.

Remark. While one hundred simulations with random variables are performed. The purpose of the simulations is only to showcase the observer-based control system and its robustness. The data should not be taken as a probability distribution statistics.

The true spacecraft inertia matrix is generated as follows. First, the true principal inertia matrix is computed according to

$$
J_{p}=\left[\begin{array}{ccc}
J_{x}+p_{x} & 0 & 0 \\
0 & J_{y}+p_{y} & 0 \\
0 & 0 & J_{z}+p_{z}
\end{array}\right]
$$

where $p_{x}, p_{y}, p_{z}$ are generated using MATLAB's "randn" random number generator, each with standard deviation of $3 \times 10^{-5} \mathrm{~kg} \cdot \mathrm{m}^{2}$. This corresponds to approximately $5 \%$ uncertainty (3-sigma) in the principal inertias. Then, the true spacecraft inertia matrix is obtained as

$$
J_{t}=C\left(q_{J}\right) J_{p} C\left(q_{J}\right)^{T}
$$

where $q_{J}=\left(q_{v J}, q_{4 J}\right), q_{v J}$ is generated with MATLAB's "randn" random number generator, with standard deviation of $5 \times 180 /(6 \pi)$ in each component and $q_{4 J}=\sqrt{1-q_{v J}^{T} q_{v J}}$. Consequently, $q_{J}$ is a unit quaternion representing a 5 degree (3-sigma) random rotation of the principal axes frame. It should be noted that $J_{t}$ is only used in the numerical propagation of the true attitude dynamics. The observer-based controller uses the nominal inertia matrix $J$. 
The initial true attitude is obtained as $q_{v}(0)=a \sin (\phi / 2), q_{4}(0)=\cos (\phi / 2)$, where $a \in \mathbb{S}^{2}$ is generated using MATLAB's "randn" function followed by normalization, and $\phi \in \mathbb{R}$ is generated using MATLAB's "randn" function, with standard deviation of $\pi / 3$, corresponding to an initial attitude error of 180 degrees (3-sigma). The initial true inertial angular velocity $\omega(0)$ is generated using MATLAB's "randn" function, with standard deviation of $5 \times \pi /(3 \times 180)$ corresponding to an initial angular velocity of 5 degrees/second (3-sigma). The initial estimated inertial angular velocity $\hat{\omega}(0)$ is generated using MATLAB's "randn" function, with standard deviation of $1.4 \times \pi /(3 \times 180)$ corresponding to an initial estimated angular velocity of 1.4 degrees/second (3-sigma). Finally, the initial estimated attitude quaternion $\hat{q}(0)$ is taken as the initial attitude quaternion measurement $q_{m}(0)$ as generated in $(4.81)$.

For clarity in presenting the results of all 100 simulations, the attitude error is presented as the principal angle of rotation corresponding to $\epsilon$, which is given by

$$
\phi=2 \sin ^{-1}\left(\left\|q_{v}\right\|\right)
$$

The angular velocity error is presented as $\|\omega\|$. Figure 4.4 shows the resulting attitude and angular velocity responses. It can be seen that in all cases, they converge to neighborhoods of zero, even with very large initial attitude errors. These results demonstrate that even though the theoretical guarantee of ultimate boundedness for the proposed observer-based controller is local and the control is continuous and measurements are available without measurement noise, when implemented under realistic conditions in a sampled-data manner the control law retains the ultimate boundedness property even for large initial conditions.

\subsection{Chapter Summary}

This chapter investigated the velocity-free attitude control problem for the inertial attitude stabilization of underactuated rigid spacecraft in circular orbits using two 

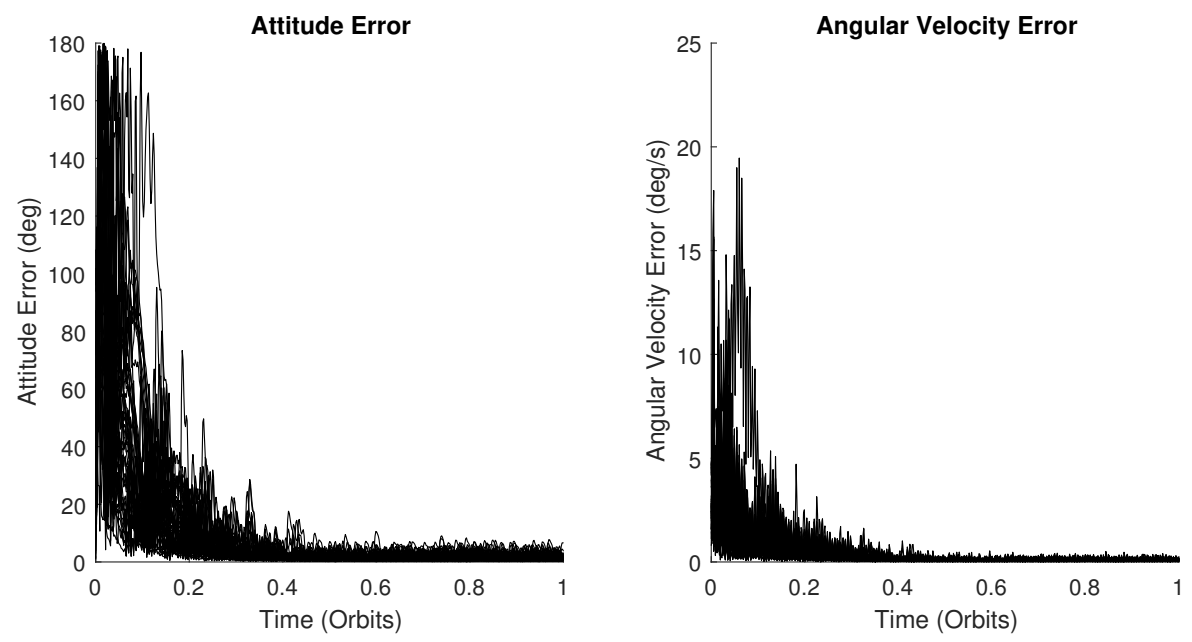

FIGURE 4.4: Attitude and angular velocity responses with inertia matrix uncertainties and disturbances

torques. Utilizing the homogeneity of systems, a velocity-free time-varying continuous feedback controller is formulated. A proposed observer-based control law combining a sliding mode control law with a observer is used to estimate the spacecraft angular velocity. A rigorous analysis has been presented to demonstrate that proposed observer-based control laws, both the reduced nominal closed-loop system and full nominal closed-loop system, are locally ultimately bounded. Numerical examples have been presented of a sampled-data implementation of the proposed observer-based control law that demonstrate robustness to uncertainties in the spacecraft inertia matrix, non-zero orbital eccentricity and measurement noise, as well as convergence for large initial attitude errors. 


\section{CHAPTER 5}

\section{Model Predictive Control of}

\section{Underactuated Spacecraft}

The space system of study is a small satellite equipped with two reaction wheels that provide two control torques. This chapter proposes the MPC-based strategy to stabilize the spacecraft's attitude with a pair of reaction wheels under the assumption of zero total system angular momentum. The attitude stabilization of a spacecraft using two torques supplied by reaction wheels aligned about the principal axes of the spacecraft is investigated in this chapter. Unlike other methods, MPC allows for the direct incorporation of practically important constraints such as spacecraft pointing, angular velocity, and wheel rate constraints. Unlike the majority of MPC applications to attitude control in the literature, which use an approximate discretized dynamics model, the exact continuous dynamics model is utilized in the MPC formulation. Numerical examples demonstrate the efficacy of the proposed approach.

\subsection{Introduction}

The stabilization of an underactuated spacecraft is complex, as such system does not have a controllable linearization, and is not feedback linearizable. This becomes further problematic when stabilizing using internal torque actuators. This chapter extends the inertial attitude control problem of Chapter 4. It deals with finding control actions using optimal control theory and model predictive control to effectively stabilize the underactuated rigid-body spacecraft system. 
Stabilization using internal torque actuators such as momentum-exchanging devices like reaction wheels (RWs) or control moment gyroscopes (CMGs) can achieve better precision than external actuators such as rockets or thrusters [Petersen, Leve, and Kolmanovsky, 2017b]. The comparative study between actuators is not outlined in this chapter. There are several drawbacks in using internal torque actuators, namely the total angular momentum in the spacecraft system must be conserved. The complete dynamics of the spacecraft with two reaction wheels is not controllable [Krishnan, McClamroch, and Reyhanoglu, 1995]. With the assumption of zero total angular momentum, the spacecraft dynamics is locally controllable at any equilibrium attitude in finite time but cannot be asymptotically stabilized to any equilibrium attitude using time-invariant continuous feedback.

However, by using model predictive control (MPC), we can generate control laws that can be used to stabilize the attitude for an underactuated spacecraft. The study of model predictive control (MPC) has gained much attention in the recent years due to its ability to generate feedback controllers with enforced constraints on dynamical continuous-time systems. MPC has a rich history [Mayne, 2014b; Qin and Badgwell, 2003] in academic literature and in the process industries, where proven to be more effective in comparison other multivariable control alternatives. MPC optimizes a control sequence while minimizing a specified cost function over a finite horizon. Analysis of its stability and optimality can be found in Mayne [Mayne, 2014a]. Application of MPC in underactuated systems by design or by result of on-board failures is not well-addressed. When there are fewer number of available torques than degrees-of-freedom, the spacecraft degrades to to an underactuated nonholonomic system that results in highly nonlinear equations of motion, which can result in uncontrollability of the equations (kinematics and dynamics) of the spacecraft[Crouch, 1984]. See Fontes [Fontes, 2001] for construction of a stabilizing nonlinear MPC for time-varying nonlinear system with input constraint. Furthermore, the system equations are not feedback linearizable which when linearized, possesses an uncontrollability linearization.MPC had been widely used in literature to solve attitude problems. Petersen et al. [Petersen, Leve, and Kolmanovsky, 2017a] proposed the use MPC to control a spacecraft with two reaction wheels. Utilizing zero momentum strategy and approximate model of the system dynamics, a 
state-discontinuous feedback locally stabilizing control law for pointing equilibrium was generated via MPC while satisfying constraints. It was proven that the attitude equations can be reduced in the case where angular momentum is zero [Krishnan, McClamroch, and Reyhanoglu, 1995]. This implied the possibility of rest-to-rest maneuvers as the reduced equations are small-time locally controllable (STLC). Gui et al. [Gui, Vukovich, and S. Xu, 2015] investigated the equilibrium STLC of combined dynamics of spacecraft with three or more independent-type CMGs in pyramid and roof-type configurations. Assuming constant inertial angular momentum and no external torques the spacecraft was stabilized via time-invariant piecewise continuous feedback laws with controllability proofs while imposing singular avoidance constraints.

\subsection{Problem Formulation}

\subsubsection{Attitude kinematics and dynamics}

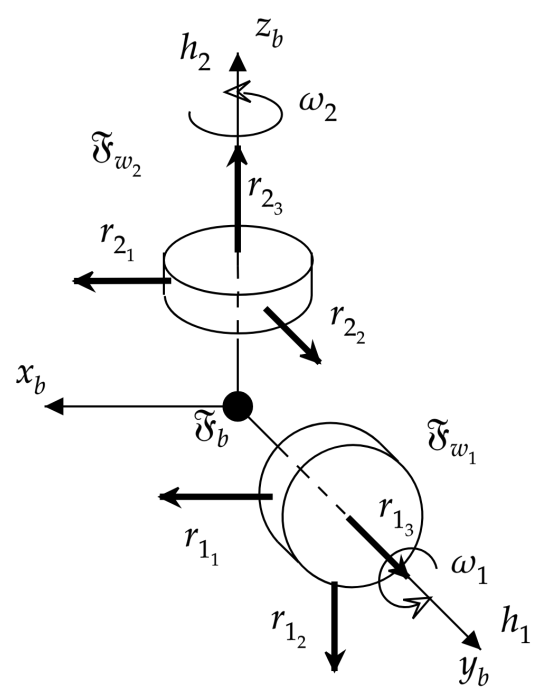

FIGURE 5.1: Two-RW system topology.

Consider a spacecraft with equipped with two reaction wheels (RW) with coordinate topology shown in Figure 5.1. The frame of the $i$ th reaction wheel is represented by the orthogonal set $\mathfrak{F}_{w_{i}}=\left\{r_{i_{1}}, r_{i_{2}}, r_{i_{3}}\right\}$, for $i=1,2$, where $r_{i_{1}}$ and $r_{i_{3}}$ are set to aligned with the rigid-body spacecraft body frame and $r_{i_{2}}=r_{i_{3}} \times r_{i_{1}}$, to complete the righthanded triad. More specifically, we align the reaction wheel's spin axis $\left(r_{i_{3}}\right)$ with one 
of the spacecraft's principal axis with reaction wheel angular velocity, $\omega_{i} \in \mathbb{R}$ for $i=1,2$. Without the loss of generality, assume that the reaction wheels are aligned in the body principal axes, $y_{b}$ and $z_{b}$, making the spacecraft $x_{b}$-axis an unactuated axis. Thus, classifying this system as underactuated. Let the angular momentum of the reaction wheels be denoted by $h$ and given by the equation:

$$
h_{i}=J_{w} \omega_{i}, i=1,2
$$

The total angular momentum contribution of the reaction wheel array, $h$, with inertia $J_{w}$ can be expressed as

$$
h=A_{w} \sum_{i=1}^{n} h_{i}=J_{w} \omega_{1} r_{1_{3}}+J_{w} \omega_{2} r_{2_{3}} .
$$

The total angular momentum of the spacecraft-RW system with respect to $\mathfrak{F}_{I}$, written in $\mathfrak{F}_{b}$ is written as

$$
H=J \omega+A_{w} h,
$$

where

$$
A_{w}=\left[\begin{array}{ll}
0 & 0 \\
1 & 0 \\
0 & 1
\end{array}\right],
$$

is RW configuration matrix, $J \in \mathbb{R}^{3 \times 3}$ is the spacecraft inertia matrix.

When no external torque $(H=0)$ act on the spacecraft (this assumption is realistic after detumbling has occured), $H$ will remain a constant, and $\omega$ can be solved directly from (5.3) and the zero-momentum restriction imposes the following:

$$
\omega=-J^{-1} A_{w} h
$$

Remark. If the spacecraft's angular momentum is initially non-zero $(H \neq 0)$, then due to the conservation of angular momentum, regardless of its new attitude vector, the angular momentum will remain non-zero and the spacecraft cannot be stabilized with respect to the origin without some form of external torque. 
Assuming the angular momentum contribution of the reaction wheel in (5.3) is negligible [Kurokawa, 2007; S. Bhat and Tiwari, 2009], then the equations of motion that describes the dynamics of the system that evolves in the angular momentum level set diffeomorphic to $\mathbb{R}^{3} \times \mathbb{T}^{n}$ is given by:

$$
\begin{aligned}
& \dot{p}=-S(p) J^{-1} A_{w} h, \\
& \dot{h}=u,
\end{aligned}
$$

where

$$
S(p)=\frac{1}{4}\left(\left(1-p^{T} p\right) I+2 p p^{T}+2 p^{\times}\right)
$$

$p=\left[p_{1}, p_{2}, p_{3}\right]^{T} \in \mathbb{R}^{3}$ denotes the Modified Rodrigues Parameters (MRPs) which describes the spacecraft orientation. $h=\left[h_{1}, h_{2}, \ldots, h_{n}\right]^{T} \in \mathbb{R}^{n}$ represents the angular momentum vector and $u=\left[u_{1}, u_{2}, \ldots, u_{n}\right]^{T} \in \mathbb{R}^{n}$ is the control input vector with $\left|u_{i}\right| \leq u_{\max }$ for $i=1,2, \ldots, n$. The rotation matrix corresponding to the MRPs, $p$ is given by

$$
C(p)=\frac{I+8 p^{\times} p^{\times}-4\left(1-p^{T} p\right) p^{\times}}{\left(1+p^{T} p\right)^{2}}
$$

Let the state vector of (5.6) and be denoted by $x=(p, h) \in M$ with configuration manifold $M=\mathbb{R}^{3} \times \mathbb{R}^{n}$. Then, the dynamics represented by (5.6) can be written in control system form as:

$$
\dot{x}=f_{0}(x)+\sum_{i=1}^{n} u_{i} f_{i}(x), \quad x \in M, \quad u_{i} \leq u_{\max }
$$

where the terms $f_{0}(x)=\left(-S(p) J^{-1}\left(A_{w} h\right), 0\right)$ and $f_{i}(x)=\left(0, e_{i}\right)$ are called the drift vector field and control vector fields, respectively. Here, $e_{i}$ denotes the $i$ th standard basis vector in $\mathbb{R}^{n}$.

\subsubsection{Optimal control}

Recall in Chapter 2, that the optimal control problem is is the minimization problem for $J\left(q(t), u(t), t_{0}, t_{f}\right)$ with control constraints and fixed boundary conditions under the assumption that the solution to the minimization exists in the Sobolev space denoted by $W^{m_{x}, \infty} \times W^{m_{u}, \infty}$ for $m_{x} \geq 1$ and $m_{u} \geq 0$. Given all admissible control 
$u=u(t)$ in $t \in\left[t_{0}, t_{f}\right]$, find the corresponding solution $x^{*}(t)$ of the problem that minimizes the cost functional $J(x(t), u(t), t)$ and satisfying the boundary condition:

$$
\min . J\left(x(t), u(t), t_{0}, t_{f}\right)=\phi\left[x_{f}, t_{f}\right]+\int_{t_{0}}^{t_{f}} \mathcal{L}[x(t), u(t), t] d t
$$

such that

$$
\begin{aligned}
\dot{p} & =-S(p) J^{-1} A_{w} h, \\
\dot{h} & =u, \\
x\left(t_{0}\right) & =x_{0} \\
x\left(t_{f}\right) & =x_{f} \\
h(x) & \leq g(x), \\
u_{\min } & \leq u(t) \leq u_{\max }
\end{aligned}
$$

where the term $h(x) \leq g(x)$ describes the path constraints of the problem described by (5.10). The optimal control problem is the minimization problem for (5.10) with control constraints and fixed boundary conditions. The next step is to discretize the dynamics described by (5.9). In this chapter, the optimization problem was discretized using pseudospectral discretization method, which is described next.

\subsubsection{Pseudospectral discretization}

In this chapter we use pseudospectral optimal control theory (Legendre pseudospectral method) to solve the underactuated spacecraft attitude stabilization. Pseudospectral optimal control theory is a spectral-based algorithm for solving nonlinear optimization control problems. The optimal trajectory is computed using transformation of the physical domain to the computational domain, interpolate using Lagrange polynomial interpolation, weighted differentiation, and weighted integration [I. Ross and Karpenko, 2012]. Much of foundation of pseudospectral optimal control theory, a theoretical-computational framework for solving an optimal problem, is the direct consequence of the Stone-Weierstrass approximation theorem. The Weierstrass approximation theorem states that a continuous real-valued function on 
a compact interval can be uniformly approximated by a set of polynomial sequence. The theorem is stated as follows:

Theorem 5.1 (Stone-Weierstrass (I. Ross and Karpenko, 2012)). Let $\mathbb{R} \supset\left[t_{0}, t_{f}\right] \Rightarrow$ $x(t) \in \mathbb{R}$ be a continuous function. Then, there exists a set of algebraic polynomial sequence $\mathfrak{W}=\left\{t \rightarrow x^{N}\right\}_{N=0}^{\infty}$ such that,

$$
\lim _{N \rightarrow \infty} \sup _{t \in\left[t_{0}, t_{f}\right]}\left\|x(t)-x^{N}(t)\right\|=0 .
$$

Since, the optimal state trajectory, $x^{*}(t)$, is assumed to exist in the Sobolev space $\left(\mathfrak{W}^{m_{x}, \infty} \times \mathfrak{W}^{m_{u}, \infty}\right)$ - the optimal solution space, it then follows that there exist a polynomial sequence $\mathfrak{W}$ that converges to $x^{*}(t)$ in the function space, $L^{\infty}$. The feasibility of the generated optimal control can be confirmed by numerical integration of the continuous dynamics of the underactuated spacecraft system using 4 th order Runge-Kutta routine.

\section{Time-domain transformation}

For a finite-horizon optimal control problem, the physical time domain $t \in\left[t_{0}, t_{f}\right]$ is mapped by affine transformation into computational domain of $\tau \in[-1,1]$ using the following,

$t=\Psi(\tau)=\left(\frac{t_{f}+t_{0}}{2}\right)+\left(\frac{t_{f}-t_{0}}{2}\right) \tau \leftrightarrow \tau=\Psi^{-1}(t)=\left(\frac{2}{t_{f}-t_{0}}\right) t-\left(\frac{t_{f}+t_{0}}{t_{f}-t_{0}}\right)$

with derivative

$$
\frac{d \tau}{d t}=\left(\frac{t_{f}-t_{0}}{2}\right)
$$

Equations (5.11) and (5.12) are used to the computation of the Legendre-GaussLobatto (LGL) quadrature node points spacing as seen in Figure 5.2.

\section{Lagrange polynomial interpolation}

Let the set of $N$ LGL node points, $t_{i} \in \mathbb{R}$, on the interval $[-1,1]$ be denoted by $\chi=\{t: i=0, \cdots, N\} \in \mathbb{R}^{N}$. Then, the corresponding Lagrange interpolant can be 


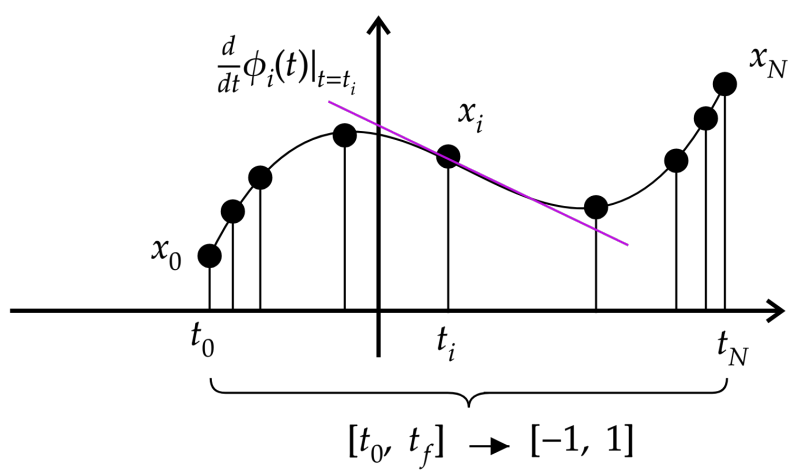

FIGURE 5.2: time domain transformation into computational domain with distinct node points and the corresponding Lagrange interpolation polynomial.

written as

$$
x^{N}(t)=\sum_{i=0}^{N} \phi_{i}(t) x_{i} \quad-1 \leq t \leq 1
$$

where $\phi_{i}(t)$ is the $N$-th order Lagrange interpolating polynomial is given by

$$
\phi_{i}(t)=\prod_{j=0, j \neq i}^{N} \frac{t-t_{j}}{t_{i}-t_{j}}
$$

the numerator of (5.14) is zero except when $j=i$ at each LGL node point, $t_{i}$. When $j=i$, Equation (5.14) satisfies the Kronecker relationship $\phi_{i}\left(t_{k}\right)=\delta_{i k}$, where

$$
\delta_{j k}\left(t_{k}\right)= \begin{cases}1, & j=i \\ 0, & j \neq i\end{cases}
$$

Remark. Using Equation (5.13), the optimal control polynomial can be interpolated and is given by

$$
u^{N}(t)=\sum_{i=0}^{N} \phi_{i}(t) u_{i} \quad-1 \leq t \leq 1
$$

\section{Differentiation and integration}

The derivative at node points of the $\mathrm{N}$-th order Lagrange interpolating polynomial is given by

$$
\left.\frac{d x^{N}(t)}{d t}\right|_{t=t_{i}}=\sum_{i=0}^{N} D_{j i} x_{i}
$$


where $D_{j i}$ is the differentiation matrix given by

$$
D_{j i}=\left.\frac{d \phi_{i}(t)}{d t}\right|_{t=t_{i}} .
$$

Similarly, the integration at node points of the $N$-th order Lagrange interpolating polynomial is given by

$$
\int_{-1}^{1} x^{N}(t) d t=\sum_{i=0}^{N} \int_{-1}^{1} \phi_{i}(t) d t x_{i}
$$

\subsubsection{Model predictive control}

Let the generic cost function be:

$$
J\left(x\left(t_{k}\right), u_{k}(\cdot), t_{0}, t_{f}\right)=\int_{t_{k}}^{t_{k}+T} \ell(x, u) d \tau
$$

where $x\left(t_{k}\right)=\left(p_{k}, \omega_{k}\right), t_{k}$ is the sample instants, $u_{k}(\cdot)$ is the control over the interval $\left[t_{k}, t_{k}+T\right]$ and $\ell(x, u)$ is a positive semi-definite function of $x$ and $u$ satisfying $\ell(x, u) \geq \alpha\|x\|^{2}, \alpha>0, \forall x, u$, and $\ell(0,0)=0$.

Now, consider the following system:

$$
\dot{x}=f(x, u), \quad f(0,0)=0 .
$$

We assume that (5.20) has a unique solution $x(t) \in X(t) \subset \mathbb{R}^{n}$ for a given $x\left(t_{0}\right)$, $u(\cdot) \in U(t) \subset \mathbb{R}^{m},(0,0) \in X(t) \times U(t)$. Moreover, for a given $x\left(t_{k}\right), u_{k}(t)$, $t \in\left[t_{k}, t_{k}+T\right]$, let $x_{k}(t)$ be the resulting solution over the interval $t=\left[t_{k}, t_{k}+T\right]$. Then, the end-point constraint, $x_{k}\left(t_{k}+T\right)=0$, is required.

\section{General MPC algorithm}

Given $x(t)$ at time $t_{k}$

Compute $u_{k}(t)$ for interval $t \in\left[t_{k}, t_{k}+T\right]$

Set $u(t)=u_{k}(t)$ over interval $\left[t_{k}, t_{k+1}\right)$

Repeat process at time $t_{k+1}$ with $x\left(t_{k+1}\right)=x_{k}\left(t_{k+1}\right)$ 
where we have $x(t)=x_{k}(t)$ and $u(t)=u_{k}(t)$ for all $t \in\left[t_{k}, t_{k+1}\right)$.

The next step is to compute the control $u_{k}(t)$ for the interval $\left[t_{k}, t_{k}+T\right]$. Suppose that at time $t_{k-1}$, for a given feasible $x\left(t_{k-1}\right)$ we have a feasible $u_{k-1}(t)$, ie. $x_{k-1}(t) \in X(t), u_{k-1}(t) \in U(t)$, and $x_{k-1}\left(t_{k}+T\right)=0$ for all $t \in\left[t_{k-1}, t_{k-1}+T\right]$. Define a candidate control:

$$
\bar{u}_{k}(t)=\left\{\begin{array}{cc}
u_{k-1}(t), & t \in\left[t_{k}, t_{k}+T-\Delta t\right) \\
0, & t \in\left[t_{k}, t_{k}+T-\Delta t\right)
\end{array} .\right.
$$

\subsubsection{Stability analysis}

Since the solutions are unique and $\bar{u}_{k} \equiv u_{k-1}$ on the interval $\left[t_{k}, t_{k}+T-\Delta t\right)$ and $t_{k}+T-\Delta t=t_{k-1}+T$, we have

$$
\bar{x}_{k}(t)=\left\{\begin{array}{cc}
x_{k-1}(t), & t \in\left[t_{k}, t_{k}+T-\Delta t\right) \\
0, & t \in\left[t_{k}, t_{k}+T-\Delta t\right)
\end{array} .\right.
$$

where $(x(t), u(t))=(0,0)$ is feasible, since $(0,0) \in X(t) \times U(t)$. Then, we have the following result:

$$
J\left(x\left(t_{k-1}\right), u_{k-1}(\cdot)\right)=\int_{t_{k-1}}^{t_{k}} \ell(x(t), u(t)) d t+\int_{t_{k}}^{t_{k-1}+T-\Delta t} \ell\left(\bar{x}_{k}, \bar{u}_{k}\right) d t+\int_{t_{k}+T-\Delta t}^{t_{k}+T} 0 d t
$$

Next, define $J_{k-1}=J\left(x\left(t_{k-1}\right), u\left(t_{k-1}\right)\right)$, and $J\left(x\left(t_{k}\right), \bar{u}_{k}\right)=\int_{t_{k}}^{t_{k-1}+T-\Delta t} \ell\left(\bar{x}_{k}, \bar{u}_{k}\right) d t+$ $\int_{t_{k}+T-\Delta t}^{t_{k}+T} 0 d t$, then we have $J_{k-1}=\int_{t_{k-1}}^{t_{k}} \ell(x, u) d t+J\left(x\left(t_{k}\right), \bar{u}_{k}\right)$. Choose $u_{k}(\cdot)$ to satisfy $J_{k}=J\left(x\left(t_{k}\right), u(\cdot)\right) \leq J\left(x\left(t_{k}\right), \bar{u}\left(t_{k}\right)\right)$, ie. improve on $u_{k}$ regardless of optimality. Then, we have

$$
J_{k}-J_{k-1} \leq-\int_{t_{k-1}}^{t_{k}} \ell(x, u) d t \leq-\alpha \int_{t_{k-1}}^{t_{k}}\|x\|^{2} d t
$$

or

$$
J_{k-1}-J_{k} \geq \alpha \int_{t_{k-1}}^{t_{k}}\|x\|^{2} d t
$$


Summing from $k=1$ to $m$, we have

$$
\begin{aligned}
\sum_{k=1}^{m}\left(J_{k-1}-J_{k}\right) & \geq \alpha \sum_{k=1}^{m} \int_{t_{k-1}}^{t_{k}}\|x\|^{2} d t \\
J_{0} & \geq J_{0}-J_{m} \geq \alpha \sum_{k=1}^{m} \int_{t_{k-1}}^{t_{k}}\|x\|^{2} d t .
\end{aligned}
$$

The second line of (5.26) holds for all $m \geq 0$.

Now, for a given $t \geq 0$, there exist $m \geq 0$ such that $t \leq t_{m}$. Then we have,

$$
h \int_{t_{0}}^{t}\|x\|^{2} d t \leq h \int_{t_{0}}^{t_{m}}\|x\|^{2} d t \leq J_{0}
$$

Thus, it holds for all $t \geq 0$. Hence, $x(t) \in \mathfrak{L}_{2}$.

If $X(t), U(t)$ is required to be contained in compact sets, and that $f(\cdot, \cdot)$ be continuous in its arguments, then $\dot{x}=f(x, u)$ is bounded, meaning $x(t)$ is uniformly continuous. By Barbalat's Lemma, $\lim _{t \rightarrow \infty} x(t)=0$.

Remark. Since $x(t)=x_{k}(t), u(t)=u_{k}(t)$ for all $t \in\left[t_{k}, t_{k+1}\right)$ and $x_{k}, u_{k}$ are feasible by design, then $(x(t), u(t)) \in X(t) \times U(t)$, for all $t \geq 0$. The feasibility of this method depends the given initial condition $\dot{x}\left(t_{0}\right)$, a feasible $u_{0}(t), t \in\left[t_{0}, t_{0}+T\right)$ needs to be found. If it exists, then by previous analysis, each subsequent problem remains feasible.

\subsection{Numerical Simulations}

This section presents a numerical simulation to demonstrate the performance of the proposed MPC and the numerical problem proposed in this section is solved using $\mathrm{DIDO}^{\complement}$, a MATLAB $^{\circledR}$ toolbox for solving optimal control problems [I. Ross, 2015]. The DIDO $^{\complement}$ program implements a fast spectral algorithm based on pseudospectral optimal control theory [I. Ross and Karpenko, 2012; Gong, Fahroo, and I. Ross, 2008], where all functions are expanded to an infinite series of special basis function and truncated to a convergence tolerance. DIDO ${ }^{\complement}$ have been successfully used in several practical examples, which attained reputable results [Fahroo and I. M. Ross, 2013; Hurni, Sekhavat, and I. Ross, 2010; Karpenko et al., 2012; Bedrossian et al., 2009]. The spacecraft parameters are taken from [Godard, K. Kumar, and A. Zou, 2013]. 
The spacecraft is in a Keplerian orbit with semi-major axis $a=6878 \mathrm{~km}$, and given initial conditions at perigee. The spacecraft nominal moments of inertia in the control and observer design are $J_{x}=0.0020 \mathrm{~kg} \cdot \mathrm{m}^{2}, J_{y}=0.0017 \mathrm{~kg} \cdot \mathrm{m}^{2}$, and $J_{z}=0.0015$ $\mathrm{kg} \cdot \mathrm{m}^{2}$. The initial attitude is $p_{0}=[0.3168,0.3168,0.0957]^{T}$ with a zero-momentum restriction, in which assumes detumbling has occurred. The optimization program and MPC parameters are outlined in Table 5.1. The time horizon is chosen to be 1 orbit, to match the simulation window using one hundred optimal control node points for better interpolation performance. The MPC control window is generally chosen as a one-tenth of the orbital period. The dynamics of the spacecraft is integrated using 4th order Runge-Kutta with standard step size of 0.1 second. Due to

TABLE 5.1: MPC Parameters

\begin{tabular}{lcc}
\hline Parameter & Parameter & Value \\
\hline \hline Time Horizon & $T$ (orbit) & 1.0 \\
Number of Nodes & $N$ & 100 \\
MPC Control Window & $\Delta T$ (orbit) & $T / 10$ \\
Cost Function gains & {$[a, b, c]$} & $\left(10^{2}\right)[1,1,0.1]$ \\
\hline
\end{tabular}

computational limitations, only a single orbit simulation is performed and optimal candidate control signals generated for ten control windows can be seen in Figure $5.3\left(t_{i}\right.$, for $\left.i=0, \cdots, 9\right)$ with 0.1 orbit spacing. The control signals are then interpolated and used as control input to the underactuated spacecraft plant. It can be seen from Figure 5.4, that a new control trajectory is sequentially generated, for example, at $t=0.1$ and $t=0.2$ orbits, respectively. From Figures 5.4, 5.6, and 5.7, the applied torque is $5.672 \times(10)^{-6} \mathrm{Nm}$. As seen in Figure 5.5, by $t=0.9$ orbit the optimal control solver has generated ten interpolated control signals for the MPC. The MRP and momentum response of continuous plant are given by Figures 5.8 and 5.9, respectively, where the underactuated rigid-body spacecraft system is stabilized from a rest-to-rest maneuver within $t=0.3$ orbit with steady-state bound of $\pm 2 \times(10)^{-3}$ in the MRP vector. 


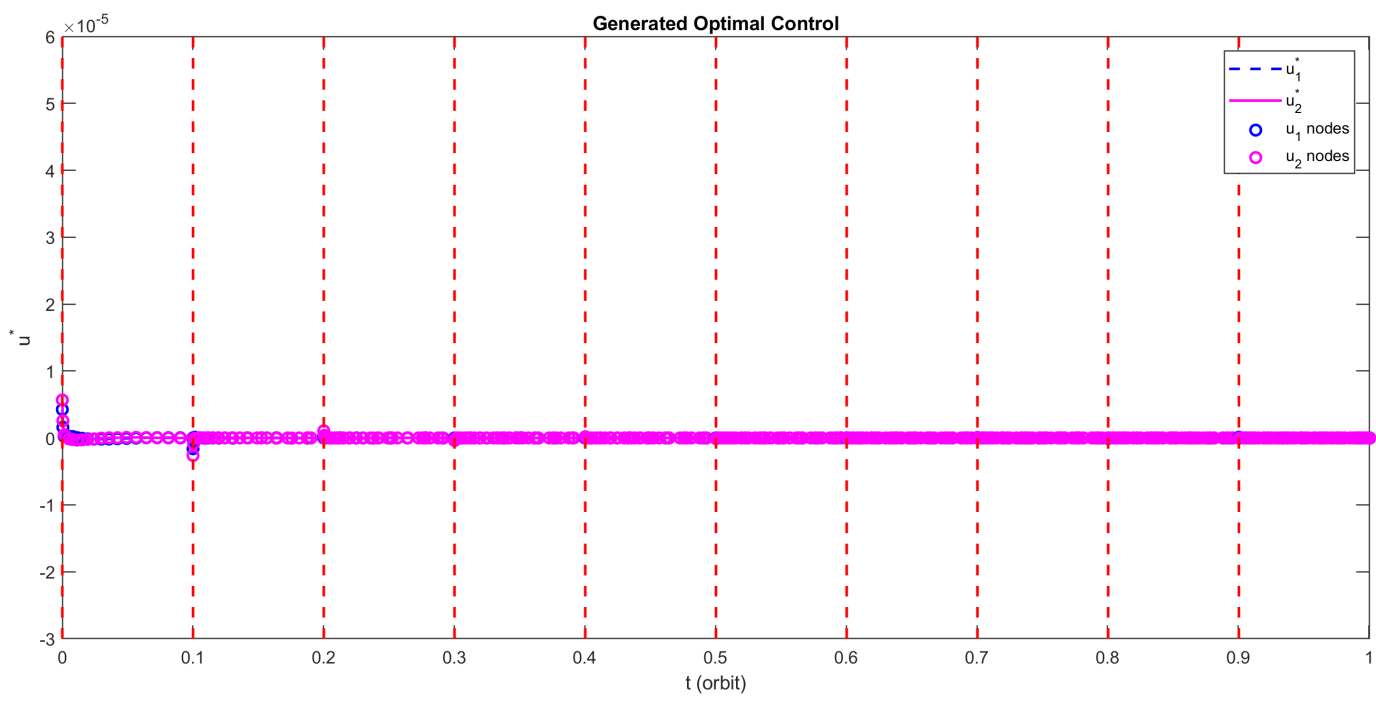

FIGURE 5.3: Optimal MPC Control Interpolation.

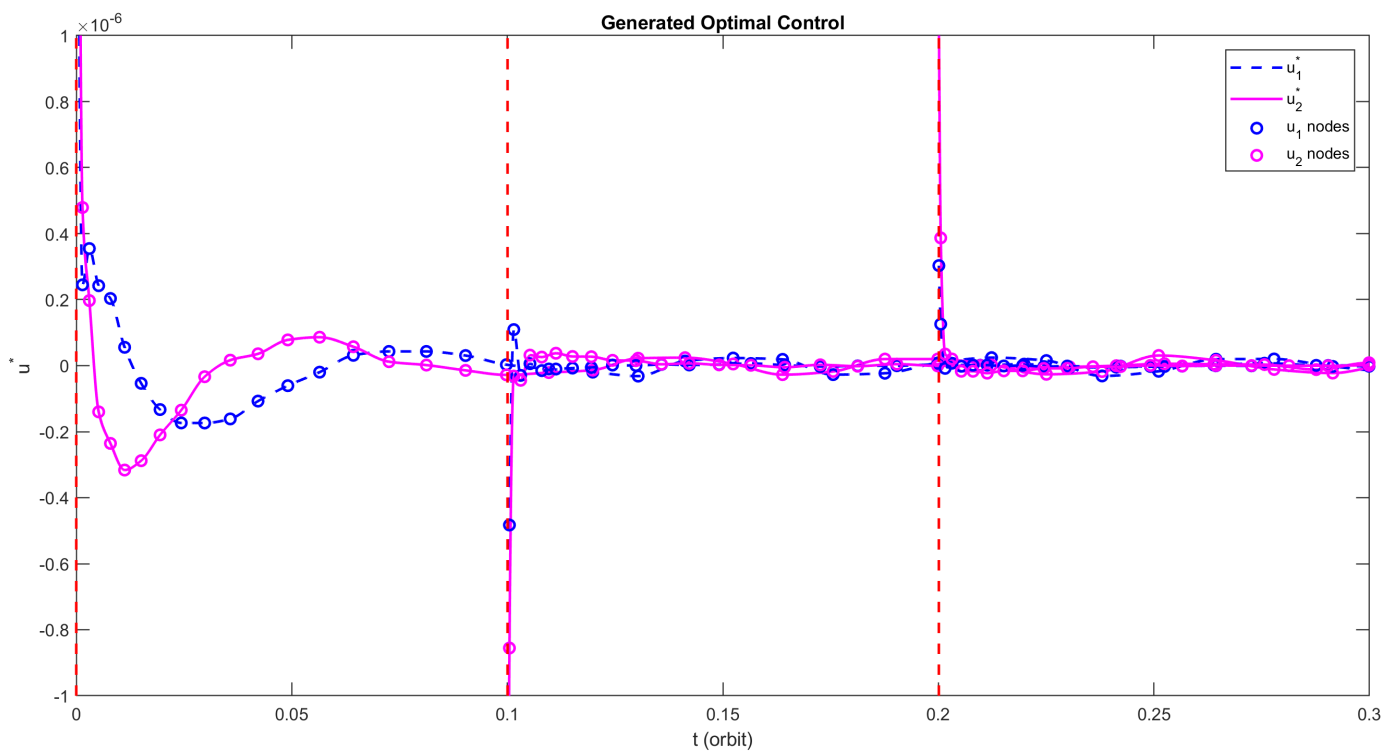

FIGURE 5.4: Optimal MPC Control Interpolation: $0 \leq t \leq 0.3$ orbits.

\subsection{Chapter Summary}

In this Chapter, the optimal control of an underactuated spacecraft with two reaction wheels using model predictive control was examined. The continuous dynamics model was used to generate an optimal control sequence using pseudospectral optimal control theory and interpolated using Lagrange polynomial interpolation. The effectiveness of the MPC was numerically simulated for small underactuated spacecraft where rest-to-rest stabilization was achieved in 0.3 orbit with a maximum 


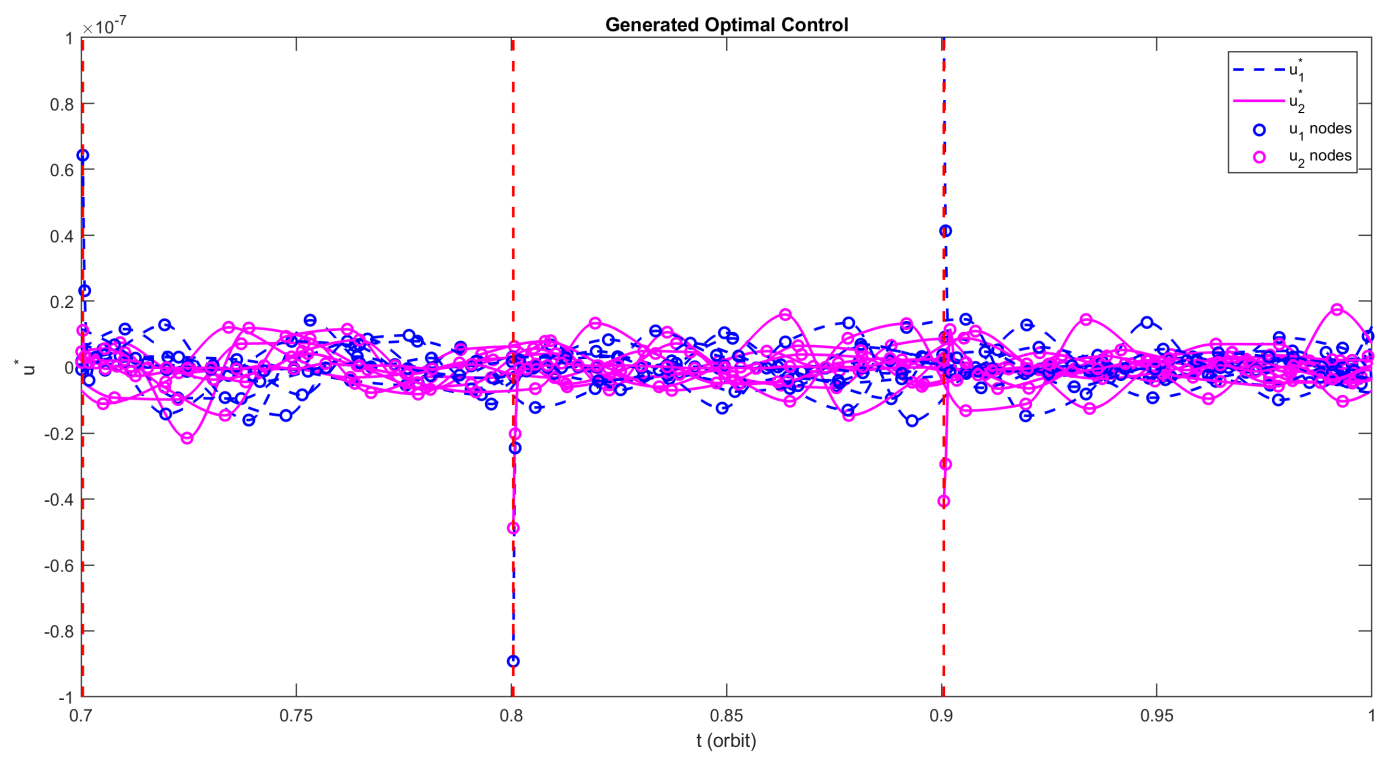

FIGURE 5.5: Optimal MPC Control Interpolation: $0.7 \leq t \leq 1.0$ orbits.

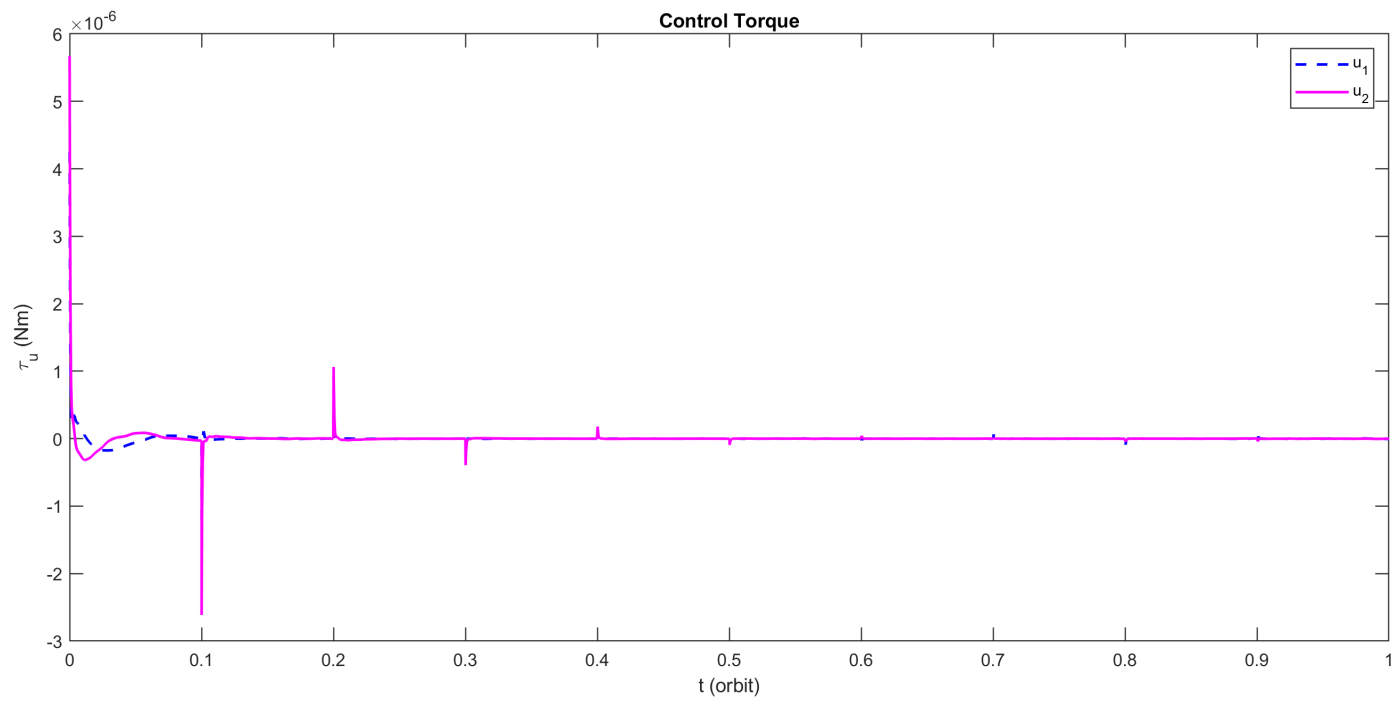

FIGURE 5.6: MPC continuous dynamics control signals.

control torque of no greater than $(10)^{-5} \mathrm{Nm}$. 


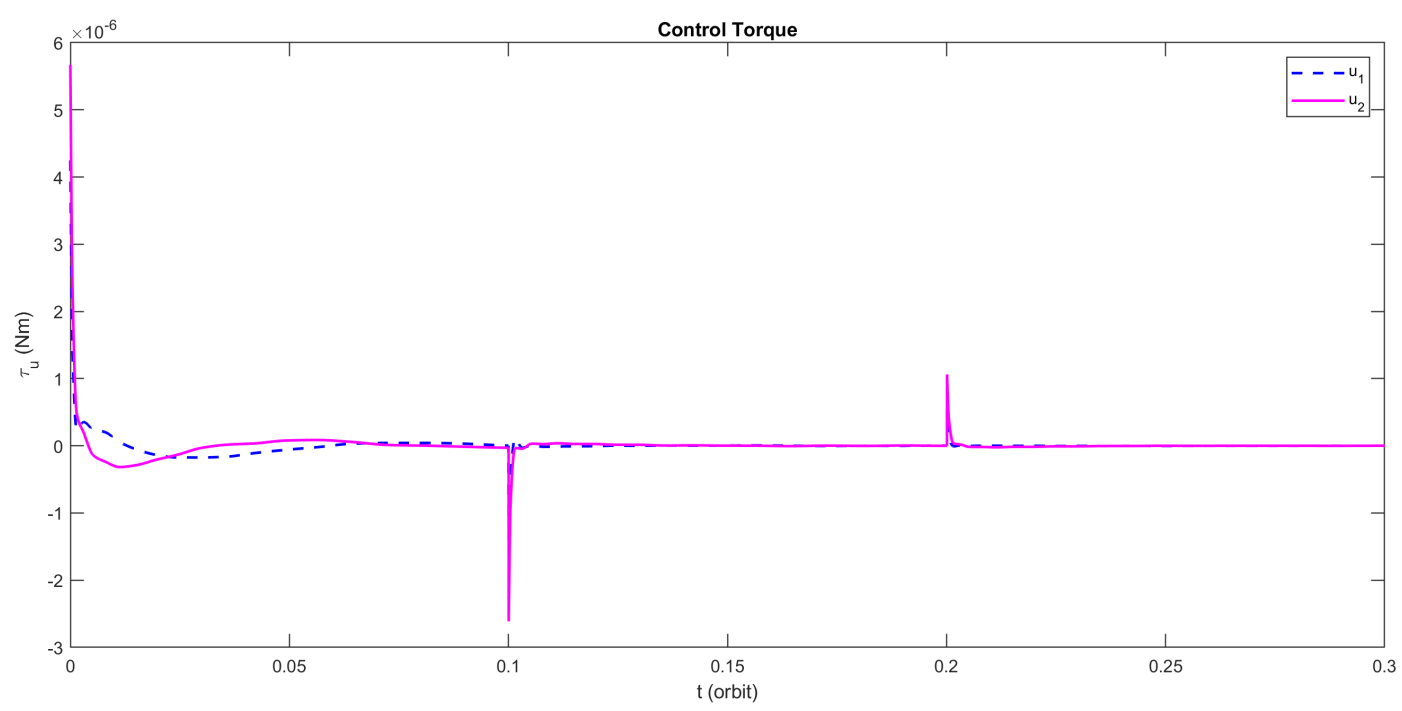

FIGURE 5.7: MPC continuous dynamics control signals at $0 \leq t \leq 0.3$ orbit.

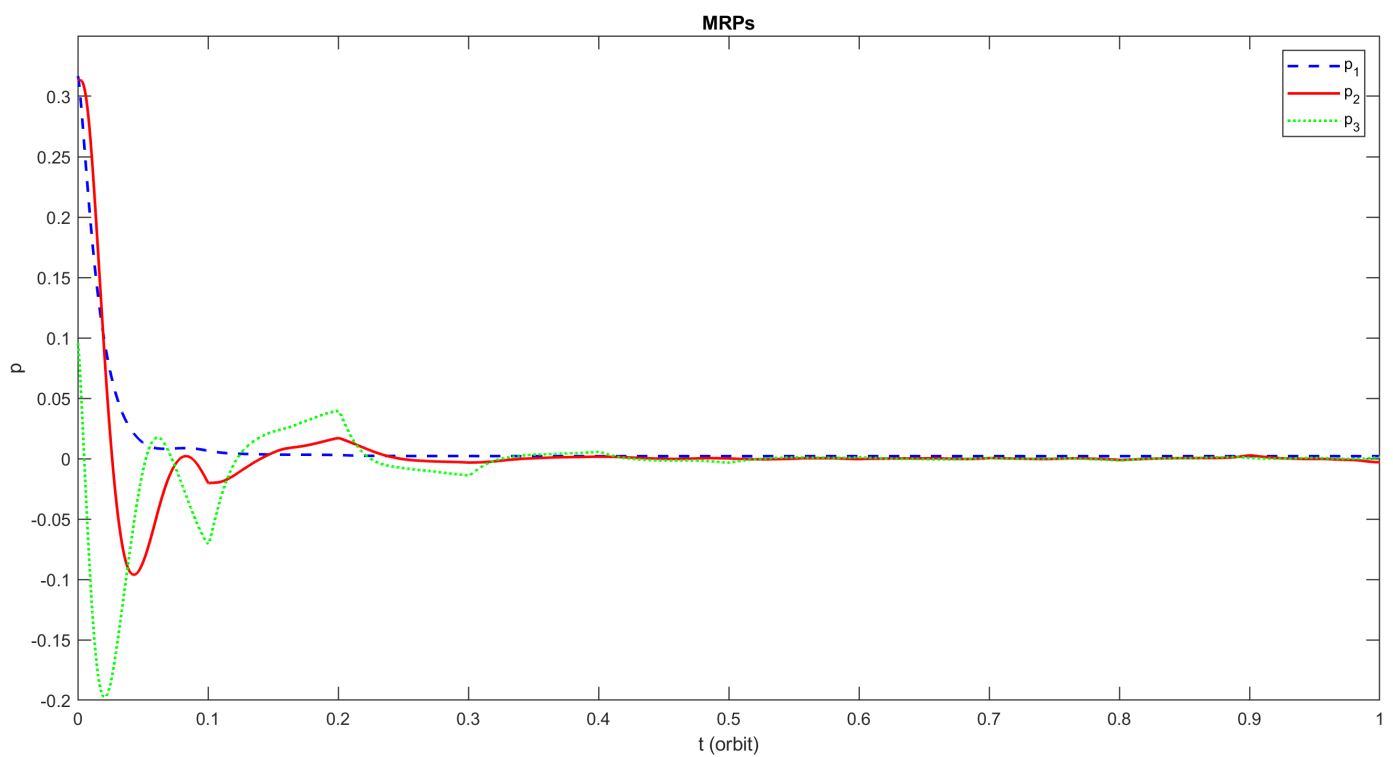

FIGURE 5.8: MRP response. 


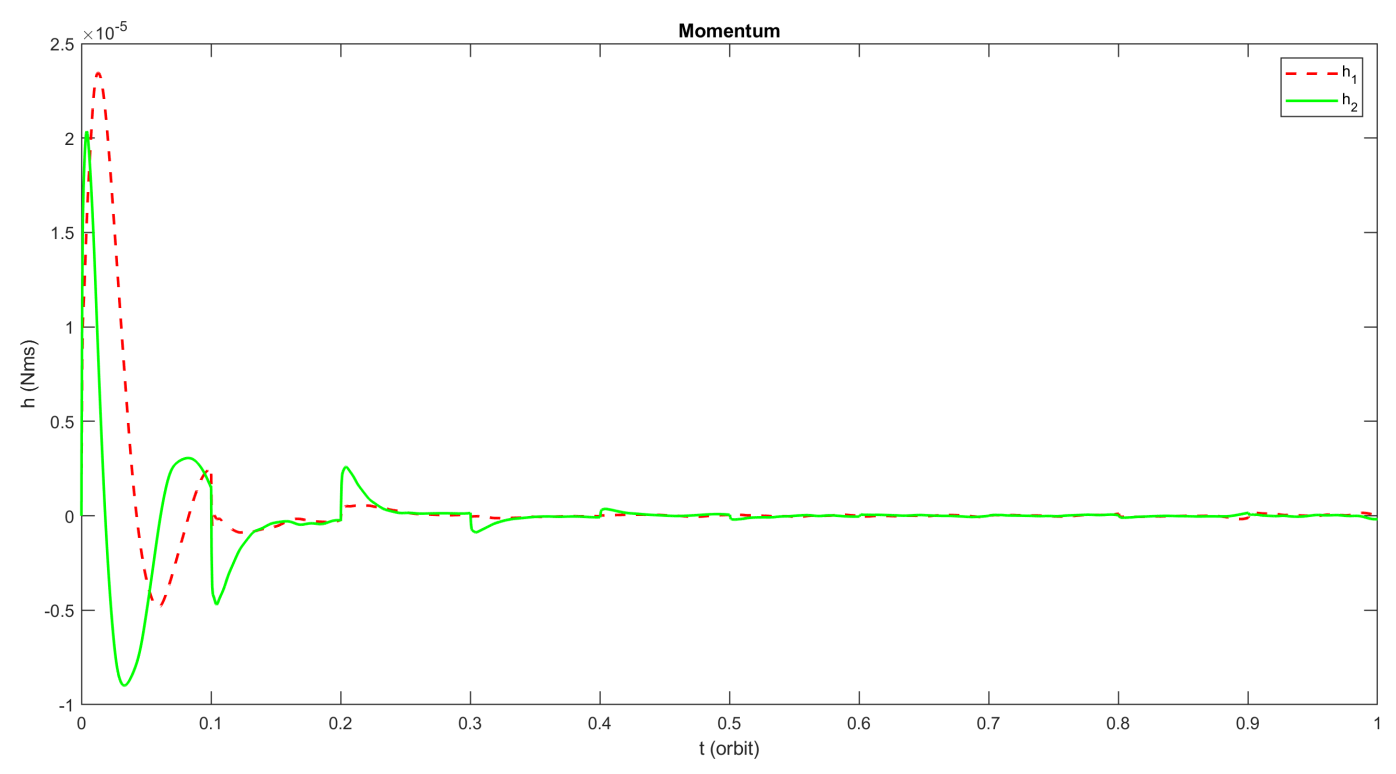

FIGURE 5.9: Angular momentum response. 


\section{CHAPTER 6}

\section{Concluding Remarks}

\subsection{Contributions}

This section outlines the key contributions of this research. These contributions arise from the problem statements, proven in the chapters and summarized the key findings as follows.

1 A novel nonlinear observer-based attitude control law that achieves attitude convergence without any angular velocity measurements for a nadir-pointing underactuated rigid-body spacecraft system in the presence of time-varying disturbances, non-zero orbital eccentricity, inertia matrix uncertainties, measurement noise in a sample-data implementation. The novel control law also guarantees ultimate boundedness of all signals even with high initial errors.

2 A generalized novel time-varying attitude control law that achieves convergence in inertial attitude for the underactuated rigid-body spacecraft system. The novel control law allows design flexibility that can lead to better performance.

3 An implementation of an optimal model predictive control to an underactuated spacecraft using pseudospectral optimal control theory with continuous dynamics model to achieve stabilization for a underactuated rigid-body spacecraft system with two reaction wheels. 


\subsection{Conclusions}

The objective of this dissertation was to investigate the nonlinear control of the attitude for an underactuated spacecraft system in accordance to the problem statements and research objectives outlined in Chapter 1. In summary, control design development and rigorous stability proofs were presented in Chapter 3 for the bodyorbital frame attitude control problem, then extended to the inertial frame in Chapter 4. Thereafter, Chapter 5 presented a method of generating optimal control signals to stabilize the underactuated control system described in the previous chapters.

The velocity-free attitude control problem for a nominally nadir-pointing and inertial-pointing underactuated rigid-body spacecraft in circular orbits with actuation about only two axes were examined in 3 and 4, respectively. An observerbased control law was proposed by combining a sliding mode control law (Chapter 3) with an finite-time nnonlinear observer to estimate the spacecraft angular velocity. Where in Chapter 4, the homogeneity of systems was utilized to develop a generalized velocity-free time-varying state feedback controller that achieved globally exponentially stable with respect to the homogenous norm. A rigorous analysis was presented demonstrating that with the proposed observer-based control laws, the closed-loop system was locally ultimately bounded. Numerical examples were presented of a sampled-data implementation of the proposed observer-based control law that demonstrate robustness to uncertainties in the spacecraft inertia matrix, non-zero orbital eccentricity and measurement noise, as well as convergence for large initial attitude errors. Lastly, in Chapter 5, the Legendre pseudospectral method was used discretized the spacecraft dynamics into Legendre-Gauss-Lobatto node points, and Lagrange polynomial interpolation was applied to obtain a candidate optimal control sequence. Model predictive control was used to implement the optimal control in predefined control windows sequentially to achieve stability for rest-to-rest maneuver. 


\subsection{Future Work}

The problem of controlling the attitude of an underactuated rigid spacecraft is still pose many realistic challenges. In recent years, there have been several major advancements in the MPC theory and particularly in applications. However MPC is limited to the computation power and the accuracy of the interpolating polynomial. Increasing the number of nodes in the optimal control generation would yield better results, but at the expense of computation cost and time, which can impose practical limitations where MPC can be applied. In Chapter 5, although reaction wheels are used for torque generation, other internal torque actuators can be used, namely, in CMGs. Singularities in CMG is a problem in many applications, by using pseudospectral optimal control theory, singularities can be added as path constraints. Thus eliminating problems due to singularities. 


\section{BIBLIOGRAPHY}

Agrachev, A.A. and Y. Sachkov (2004). Control theory from the geometric viewpoint.

1st ed. Vol. 87. Encyclopedia of Mathematical Sciences. Springer-Verlag. ISBN: 354402100199 .

Aguilar-Ibanez, C., J. Mendoza-Mendoza, and J. Davila (2014). "Stabilization of the cart pole system: by sliding mode control". In: Nonlinear Dynamics, pp. 1-9.

Bedrossian, N. et al. (2009). "Zero Propellant Maneuver Guidance". In: IEEE Control Systems Magazine 29.5, pp. 53-73.

Behal, A. et al. (2002). "Nonlinear tracking control of an underactuated spacecraft". In: Journal of Guidance, Control, and Dynamics 25.5, pp. 979-985.

Bhat, S. and P. Tiwari (2009). “Controllability of spacecraft attitude using control moment gyroscopes". In: IEEE Transactions on Automatic Control 54.3, pp. 585590.

Bhat, S.P. and D.S. Bernstein (2005). "Geometric homogeneity with application to finite-time stability". In: Mathematics of Control, Signals, and Systems 17.2, pp. 101127.

Byrnes, C. and A. Isidori (1991). "On the attitude stabilization of rigid spacecraft". In: IEEE Transactions on Automatic Control 27.1, pp. 87-95.

Carmo, M. do (1976). Differential geometry of curves and surfaces. 1st ed. Prentice-Hall, Inc. ISBN: 0132125897.

Casagrande, D., A. Astolfi, and T. Parisini (2008). "Global asymptotic stabilization of the attitude and the angular rates of an underactuated non-symmetric rigid body". In: Automatica 44.7, pp. 1781-1789.

Chunodkar, A. and M. Akella (2014). "Switching Angular Velocity Observer for Rigid-Body Attitude Stabilization and Tracking Control". In: Journal of Guidance, Control, and Dynamics. 
Coverstone-Carroll, V. (1996). “Detumbling and reorienting underactuated rigid spacecraft". In: Journal of Guidance, Control, and Dynamics 19.3, pp. 708-710.

Crouch, P. (1984). "Spacecraft attitude control and stabilization: applications of geometric control theory to rigid body models". In: IEEE Transactions on Automatic Control 29.4, pp. 321-331.

Du, H. and S. Li (2012). “Semi-global finite-time attitude stabilization by output feedback for a rigid spacecraft". In: Proc. IMechE Part G: Journal of Aerospace Engineering. Vol. 227. 12. IMechE, pp. 1881-1891.

Du, H., S. Li, and C. Qian (2011). "Finite-time attitude tracking control of spacecraft with application to attitude synchronization". In: IEEE Transactions on Automatic Control 56.11, pp. 2711-2717.

Fahroo, F. and I. M. Ross (2013). “Enhancing the Practical Agility of UAVs via Pseudospectral Optimal Control". In: Recent Advances in Research on Unmanned Systems 444 , pp. 1-30.

Fontes, F. (2001). “A general framework to design stabilizing nonlinear model predictive controllers". In: Systems $\mathcal{E}$ Control Letters 42.2, pp. 127-143.

Gao, B. et al. (2013). "Stabilizing control of an underactuated 2-dimensional TORA with only rotor angle measurement". In: Asian Journal of Control 15.3, pp. 1-12.

Godard, G. (2010). "Fault Tolerant Control of Spacecraft". PhD Thesis. Ryerson University.

Godard, G. and K. Kumar (2010). “Fault tolerant reconfigurable satellite formation using adaptive variable structure techniques". In: Journal of Guidance, Control and Dynamics 33.3, pp. 969-984.

Godard, G. and K.D. Kumar (2011). “Robust attitude stabilization of spacecraft subject to actuator failures". In: Acta Astronautica 68, pp. 1242-1259.

Godard, K. Kumar, and A. Zou (2013). “A novel single thruster control strategy for spacecraft attitude stabilization". In: Acta Astronautica 86.7, pp. 55-67.

Gong, Q., F. Fahroo, and I.M. Ross (2008). “Spectral Algorithm for Pseudospectral Methods in Optimal Control". In: Journal of Guidance, Control, and Dynamics 31.3, pp. $460-471$.

Gui, H., L. Jin, and S. Xu (2015). "Small-time local controllability of spacecraft attitude using control moment gyros". In: Automatica 53.1, pp. 141-148. 
Gui, H. and G. Vukovich (2016). “Global finite-time attitude tracking via quaternion feedback". In: Systems and Control Letters 97, pp. 176-183.

- (2017). "Finite-time angular velocity observers for rigid-body attitude tracking with bounded inputs". In: International Journal of Robust and Nonlinear Control 27, pp. 15-38.

Gui, H., G. Vukovich, and S. Xu (2015). “Attitude tracking of a rigid spacecraft using two internal torques". In: IEEE Transactions on Aerospace and Electronic Systems 51.4, pp. 2900-2913.

- (2016). "Attitude stabilization of a spacecraft with two parallel control moment gyros". In: Journal of Guidance, Control, and Dynamics 39.3, pp. 724-731.

Horri, N.M. and S. Hodgarts (2003). "Attitude stabilization of an Underactuated satellite using two wheels". In: Proceedings of the 2003 IEEE Aerospace Conference. Vol. 6, pp. 2629-2635.

Huang, J. et al. (2013). “Generalised inversion based attitude control for underactuated spacecraft". In: Acta Automatica Sinica 39.3, pp. 285-292.

Hughes, P. (1986). Spacecraft attitude dynamics. Dover Publications, Inc. Chap. 1-4, 8,9. Hurewicz, W. (1952). Chapter 2: Lectures on Ordinary Differential Equations. Cambridge, Massachusetts.

Hurni, M.A., P. Sekhavat, and I.M. Ross (2010). “An Info-Centric Trajectory Planner for Unmanned Ground Vehicles". In: Dynamics of Information Systems: Theory and Applications, pp. 213-232.

Karpenko, M. et al. (2012). "First Flight Results on Time-Optimal Spacecraft Slews". In: Journal of Guidance, Control and Dynamics 35.2, pp. 367-376.

Khalil, H.K. (1998). Nonlinear Systems. 3rd Edition. Upper Saddle River, New Jersey: Prentice Hall, pp. 102-103.

Khosravian, A. and M. Namvar (2010). “Globally Exponential Estimation of Satellite Attitude using a Single Vector Measurement and Gyro". In: pp. 364-369.

- (2012). "Rigid Body Attitude Control Using a Single Vector Measurement and Gyro". In: IEEE Transactions on Automatic Control 57.5, pp. 1273-1279.

Krishnan, H., N.H. McClamroch, and M. Reyhanoglu (1995). “Attitude Stabilization of a Rigid Spacecraft Using Two Momentum Wheel Actuators". In: Journal of Guidance, Control and Dynamics 18.2, pp. 256-263. 
Kurokawa, H. (2007). "Survey of theory and steering laws of single gimbal control moment gyros". In: Journal of Guidance, Control, and Dynamics 30.5, pp. 1331-1340.

Li, H., W. Yan, and Y. Shi (2017). “Continuous-time model predictive control of under-actuated spacecraft with bounded control torques". In: Automatica 75, pp. 144-153.

Lizarralde, F. and J. Wen (1996). “Attitude Control Without Angular Velocity Measurement: A Passivity Approach". In: IEEE Trans. on Automatic Control 41.3, pp. $468-472$.

Mayne, D.Q. (2014a). “Constrained model predictive control: Stability and optimality". In: Automatica 36.6, pp. 789-814.

- (2014b). "Model predictive control: Recent developments and future promise". In: Automatica 50.12, pp. 2967-2986.

Morin, P. and C. Samson (1997). “Time-Varying Exponential Stabilization of a Rigid Spacecraft with Two Control Torques". In: IEEE Transactions on Automatic Control 42.4, pp. 528-534.

— (2009). "Control of nonholonomic mobile robots based on the transverse function approach". In: IEEE Transactions on Robotics 25.5, pp. 1058-1073.

Narikiyo, T., J. Sahashi, and K. Misao (2008). “Control of a class of underactuated mechanical system". In: Nonlinear Analysis: Hybrid Systems 2.2, pp. 231-241.

Petersen, C.D., F. Leve, and I. Kolmanovsky (2017a). “Model predictive control of an underactuated spacecraft with two reaction wheels". In: Journal of Guidance, Control, and Dynamics 40, pp. 320-332.

- (2017b). "Underactuated spacecraft switching law for two reaction wheels and constant angular momentum". In: Journal of Guidance, Control, and Dynamics 39.9, pp. 2086-2099.

Polycarpou, M. and P. Ioannou (1996). "A robust adaptive nonlinear control design". In: Automatica 32.3, pp. 432-427.

Qin, S.J. and T.A. Badgwell (2003). "A survey of industrial model predictive control technology". In: Control Engineering Practice 11.7, pp. 733-764.

Rosier, L. (1992). “Homogeneous Lyapunov Function for homogeneous continuous vector field". In: Systems and Control Letters 19.6, pp. 467-473. 
Ross, I.M. (2015). A Primer on Pontryagin's Principle in Optimal Control. Second Edition. San Francisco: Collegiate Publishers. Chap. 3.2.

Ross, I.M. and M. Karpenko (2012). “A Review of Pseudospectral Optimal Control: From Theory to Flight". In: Annual Reviews in Control 36.2, pp. 182-187.

Ruiter, A. de, C. Damaren, and J. Forbes (2013). Spacecraft Dynamics and Control: An Introduction. 1st ed. West Sussex, United Kingdom: John Wiley \& Sons, Ltd. ISBN: 9781118342367.

Salcudean, S. (1991). “A globally convergent angular velocity observer for rigid body motion". In: IEEE Transactions on Automatic Control 36.12, pp. 1493-1497.

Seo, D. and M. Akella (2007). "Separation Property for the Rigid-Body Attitude Tracking Control Problem". In: Journal of Guidance, Control and Dynamics 30.6, pp. 1569-1576.

Spong, M.W. (1995). "The swing up control problem for the Acrobot". In: IEE Control Systems 15.1, pp. 49-55.

Sun, N. and Y. Fang (2014). “An efficient online trajectory generating method for underactuated crane systems". In: International Journal Robust Nonlinear Control 24, pp. 1653-1663.

Tayebi, A. (2007). "A velocity-free attitude tracking controller for rigid spacecraft". In: pp. 6430-6434.

Thakur, D. and M. Akella (2015). “Gyro-Free Rigid-Body Attitude Stabilization Using only Vector Measurements". In: Journal of Guidance, Control, and Dynamics 38.4, pp. 811-818.

Trumpf, J. et al. (2012). "Analysis of Non-Linear Attitude Observers for TimeVarying Reference Measurements". In: IEEE Transactions on Automatic Control 37.11, pp. 2789-2800.

Tsiotras, P. and J.H. Luo (2000). “Control of underactuated spacecraft with bounded inputs". In: Automatica 36.8, pp. 1153-1169.

Xiao, B. et al. (2014). "Fault-tolerant control of spacecraft with attitude-only measurement under actuator failures". In: Journal of Guidance, Control, and Dynamics AIAA Early Edition, pp. 1-12.

Xin, X. and Y. Liu (2014). Control design and analysis for underactuated robotic systems. 1st ed. Springer. ISBN: 9781447162506. 
Xu, R. and U. Ozguner (2008). "Sliding mode control of a class of underactuated systems". In: Automatica 44.1, pp. 233-241.

Zhang, J., K. Ma, and G. Meng (2014). “Controllability analysis and attitude path planning of underactuated spacecraft systems". In: Aerospace Science and Technology 33, pp. 76-81.

Zhang, M. (2002). "Hybrid control of the pendubot". In: IEEE/ASME Transaction on Mechantronics 7.1, pp. 79-86.

Zhou, K. and J.C. Doyle (1998). Essentials of Robust Control. Upper Saddle River, NJ: Prentice Hall.

Zlotnik, D. E. and J. R. Forbes (2017). “Nonlinear Estimator Design on the Special Orthogonal Group Using Vector Measurements Directly". In: IEEE Transactions on Automatic Control 62.1, pp. 149-160.

Zou, A.M. (2014). "Finite-time output feedback attitude tracking control for rigid spacecraft". In: IEEE Transactions on Control Systems Technology 22.1, pp. 338-345. 